\title{
Total Synthesis of the Marine Natural Product Solomonamide B Necessitates Stereochemical Revision
}

K. Kashinath, ${ }^{1 \text { abt }}$ Gorakhnath R. Jachak, ${ }^{1 \text { abt }}$ Paresh R. Athawale, ${ }^{\text {lat }}$ Udaya Kiran Marelli, ${ }^{1,2 a b}$ Rajesh G. Gonnade ${ }^{3 a b}$ and D. Srinivasa Reddy*1ab

a. Division of Organic Chemistry, ${ }^{1}$ Central NMR Facility, ${ }^{2}$ Center for Materials Characterization, ${ }^{3}$ CSIR-National Chemical Laboratory, Dr. HomiBhabha Road, Pune, 411008, India.

b. Academy of Scientific and Innovative Research (AcSIR), New Delhi, India.

${ }^{\dagger}$ All three authors contributed equally.

\author{
*Corresponding author: \\ Dr. D. Srinivasa Reddy \\ CSIR-National Chemical Laboratory, \\ Division of Organic Chemistry, \\ Dr. HomiBhabha Road, \\ Pune 411008, Maharashtra, \\ India. \\ E-mail: ds.reddy@ncl.res.in
}

Tel. +912025902445

Fax. +912025902629

\section{Supporting Information}

Table of contents

page

General

Experimental procedures

Copies of ${ }^{1} \mathrm{H},{ }^{13} \mathrm{C}$ NMR, 2D NMR spectra 


\section{General:}

All reactions were carried out in oven-dried glassware under a positive pressure of argon or nitrogen unless otherwise mentioned with magnetic stirring. Air sensitive reagents and solutions were transferred via syringe or cannula and were introduced to the apparatus via rubber septa. All reagents, starting materials and solvents were obtained from commercial suppliers and used as such without further purification. Reactions were monitored by thin layer chromatography (TLC) with $0.25 \mathrm{~mm}$ pre-coated silica gel plates (60 F254). Visualization was accomplished with either UV light, Iodine adsorbed on silica gel or by immersion in ethanolic solution of phosphomolybdic acid (PMA), p-anisaldehyde, 2,4-DNP, $\mathrm{KMnO}_{4}$, Ninhydrin solution followed by heating with a heat gun for $\sim 15 \mathrm{sec}$. Column chromatography was performed on silica gel (100-200 or 230-400 mesh size). Deuterated solvents for NMR spectroscopic analyses were used as received. All ${ }^{1} \mathrm{H}$ NMR, ${ }^{13} \mathrm{C}$ NMR and 2D NMR spectra were obtained using a $200 \mathrm{MHz}, 400$ $\mathrm{MHz}, 500 \mathrm{MHz}$ spectrometer. Coupling constants were measured in Hertz. All chemical shifts were quoted in ppm, relative to TMS, using the residual solvent peak as a reference standard. The following abbreviations were used to explain the multiplicities: $\mathrm{s}=$ singlet, $\mathrm{d}=$ doublet, $\mathrm{t}=$ triplet, $\mathrm{q}=$ quartet, $\mathrm{m}=$ multiplet, $\mathrm{br}=$ broad. HRMS $(\mathrm{ESI})$ were recorded on ORBITRAP mass analyzer (Thermo Scientific, QExactive). Mass spectra were measured with ESI ionization in MSQ LCMS mass spectrometer. Infrared (IR) spectra were recorded on a FT-IR spectrometer as a thin film. Chemical nomenclature was generated using Chembiodraw ultra 14.0 Melting points of solids were measured in melting point apparatus (Buchi 565). Optical rotation values were recorded on P-2000 polarimeter at $589 \mathrm{~nm}$. 


\section{Experimental procedures:}<smiles>CSCCC(N)C(=O)O</smiles>

7

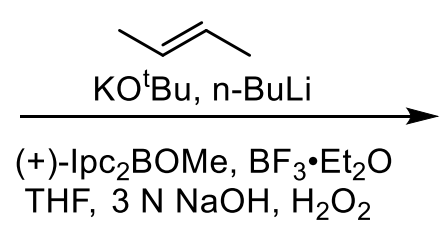

$61 \%$

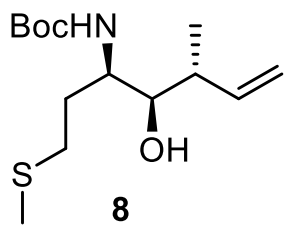

8

tert-Butyl ((3R,4R,5R)-4-hydroxy-5-methyl-1-(methylthio)hept-6-en-3-yl)carbamate (8)

(E)-2-butene ( $3 \mathrm{~mL}, 17 \mathrm{mmol}, 2$ equiv) was condensed into flask containing $\mathrm{KO}^{\mathrm{t}} \mathrm{Bu}(2.4 \mathrm{~g}, 21.5$ mmol, 1.25 equiv) in THF (20 mL) chilled to $-78^{\circ} \mathrm{C}$. After careful addition of $\mathrm{n}$-BuLi (13.4 mL, 1.6 $\mathrm{M}$ in hexanes, $21.5 \mathrm{mmol}, 1.25$ equiv) over 1 hour (maintaining internal temperature below $70{ }^{\circ} \mathrm{C}$ ), the reaction mixture was warmed to $-50{ }^{\circ} \mathrm{C}$ for $15 \mathrm{~min}$. The mixture was chilled to -78 ${ }^{\circ} \mathrm{C}$ once more, and a solution of (+)-B-methoxydiisopinocampheylborane $(25 \mathrm{~mL}, 1 \mathrm{M}$ in $\mathrm{THF}$, $25 \mathrm{mmol}, 1.45$ equiv) was added slowly over $30 \mathrm{~min}$. Next, $\mathrm{BF}_{3} \cdot \mathrm{Et}_{2} \mathrm{O}$ (4.1 mL, $29.1 \mathrm{mmol}, 1.7$ equiv) was added at $-78{ }^{\circ} \mathrm{C}$ over 30 minutes, then the solution of reagent was treated with a solution of compound $7^{1}(4.3 \mathrm{~g}, 17.1 \mathrm{mmol})$ in THF $(25 \mathrm{~mL})$. The reaction mixture was stirred at $-78{ }^{\circ} \mathrm{C}$ for $12 \mathrm{~h}$, then warmed to $-15^{\circ} \mathrm{C}$. A mixture of $3 \mathrm{~N} \mathrm{NaOH}(8.3 \mathrm{~mL})$ and $30 \%$ hydrogen peroxide $(2.9 \mathrm{~mL})$ was added dropwise to the reaction. After being heated to reflux for $1 \mathrm{~h}$, the mixture was cooled to $0{ }^{\circ} \mathrm{C}$. Concentrated the reaction mixture, diethylether $(50 \mathrm{~mL})$ was added and washed with water $(20 \mathrm{~mL})$ and brine $(20 \mathrm{~mL})$. The organic layer was dried over $\mathrm{Na}_{2} \mathrm{SO}_{4}$ and the crude material obtained after removal of the solvent was purified by column chromatography (silica gel 230-400 mesh 10\% ethyl acetate - petether) to afford 8 (3.25 g, 61\%) as colorless liquid as a single diastereomer. $[\alpha]_{\mathrm{D}}^{30}+9.38\left(c 0.45, \mathrm{CHCl}_{3}\right)$; IR $v_{\max }(\mathrm{film}): \mathrm{cm}^{-1} 3439,3017$, 2977, 1701, 1501, 1442; ${ }^{1} \mathrm{H}$ NMR (400 MHz, $\left.\mathrm{CDCl}_{3}\right): \delta 5.69(\mathrm{td}, J=9.2,18.0 \mathrm{~Hz}, 1 \mathrm{H}), 5.21$ $5.10(\mathrm{~m}, 2 \mathrm{H}), 4.83(\mathrm{~d}, J=9.5 \mathrm{~Hz}, 1 \mathrm{H}), 3.93-3.76(\mathrm{~m}, 1 \mathrm{H}), 3.24(\mathrm{~d}, J=8.6 \mathrm{~Hz}, 1 \mathrm{H}), 2.58-2.46$ (m, 2H), $2.26-2.18(\mathrm{~m}, 1 \mathrm{H}), 2.13$ (brs, 1H), 2.09 (s, 3H), $1.93-1.76(\mathrm{~m}, 2 \mathrm{H}), 1.43(\mathrm{~s}, 9 \mathrm{H}), 1.03$ $(\mathrm{d}, J=6.8 \mathrm{~Hz}, 3 \mathrm{H}) ;{ }^{13} \mathrm{C}$ NMR $\left(100 \mathrm{MHz}, \mathrm{CDCl}_{3}\right): \delta 156.1,140.8,117.2,79.2,75.7,50.3,42.3$, 33.4, 30.9, 28.4, 16.5, 15.6; HRMS calculated for $\mathrm{C}_{14} \mathrm{H}_{27} \mathrm{O}_{3} \mathrm{NNa}[\mathrm{M}+\mathrm{Na}]^{+}:$312.1604, found 312.1599 . 


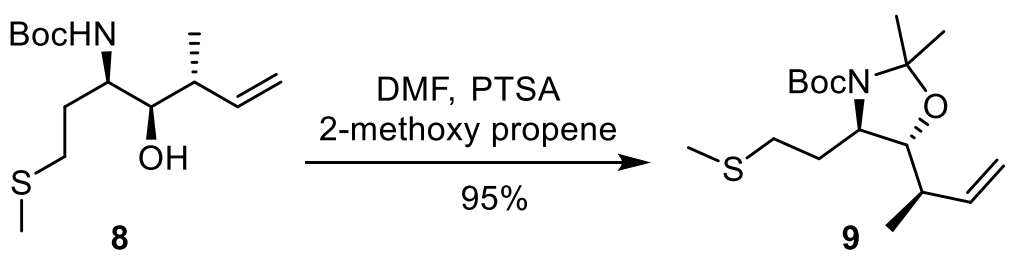

tert-Butyl (4R,5R)-5-((R)-but-3-en-2-yl)-2,2-dimethyl-4-(2-(methylthio)ethyl)oxazolidine-3carboxylate (9): To a stirred solution of compound 8 (7.0 g, $24.2 \mathrm{mmol})$, and 2-methoxypropene $(5.82 \mathrm{~mL}, 60.5 \mathrm{mmol})$ in dry DMF (20 mL) PTSA. $\mathrm{H}_{2} \mathrm{O}(93 \mathrm{mg}, 4.8 \mathrm{mmol})$ was added at $0{ }^{\circ} \mathrm{C}$ under argon atmosphere. The resulting solution was stirred at room temperature for $4 \mathrm{~h}$. The reaction was then diluted with $\mathrm{H}_{2} \mathrm{O}(5 \mathrm{~mL})$ and extracted with EtOAc $(2 \times 50 \mathrm{~mL})$. The combined organic layers were washed with cold saturated $\mathrm{NaHCO}_{3}$ solution $(15 \mathrm{~mL}), \mathrm{H}_{2} \mathrm{O}(10$ $\mathrm{mL})$, brine $(10 \mathrm{~mL})$, and evaporated in vacuo. Purification by column chromatography (silica gel 100-200 mesh 10\% ethyl acetate - pet ether) yielded compound 9 (7.53 g, 95\%) as a pale yellow color liquid. $[\alpha]_{\mathrm{D}}{ }^{30}-8.97\left(c 0.97, \mathrm{CHCl}_{3}\right)$; IR $v_{\max }\left(\right.$ film): $\mathrm{cm}^{-1} 3020,2978,1684,1597 ;{ }^{1} \mathrm{H} \mathrm{NMR}$ (400 MHz, $\left.\mathrm{CDCl}_{3}\right): \delta 5.90$ - $5.75(\mathrm{~m}, 1 \mathrm{H}), 5.11$ - $4.96(\mathrm{~m}, 2 \mathrm{H}), 3.88$ (brs, 1H), 3.62 (dd, J= 3.7, $7.8 \mathrm{~Hz}, 1 \mathrm{H}), 2.53$ - 2.28 (m, 3H), 2.10 (s, 3H), 1.92 (dd, J=6.6, $7.3 \mathrm{~Hz}, 2 \mathrm{H}), 1.55$ (brs, 3H), 1.46 (s, 12H), $1.02(\mathrm{~d}, J=6.8 \mathrm{~Hz}, 3 \mathrm{H}) ;{ }^{13} \mathrm{C} \mathrm{NMR}\left(100 \mathrm{MHz}, \mathrm{CDCl}_{3}\right): \delta 151.9,139.7,115.5,94.3$, 83.7, 80.0, 59.4, 41.4, 33.1, 32.3, 30.3, 28.4, 27.9, 27.3, 17.0, 15.5; HRMS calculated for $\mathrm{C}_{17} \mathrm{H}_{31} \mathrm{O}_{3} \mathrm{NNaS}[\mathrm{M}+\mathrm{Na}]^{+}:$352.1917, found 352.1914.

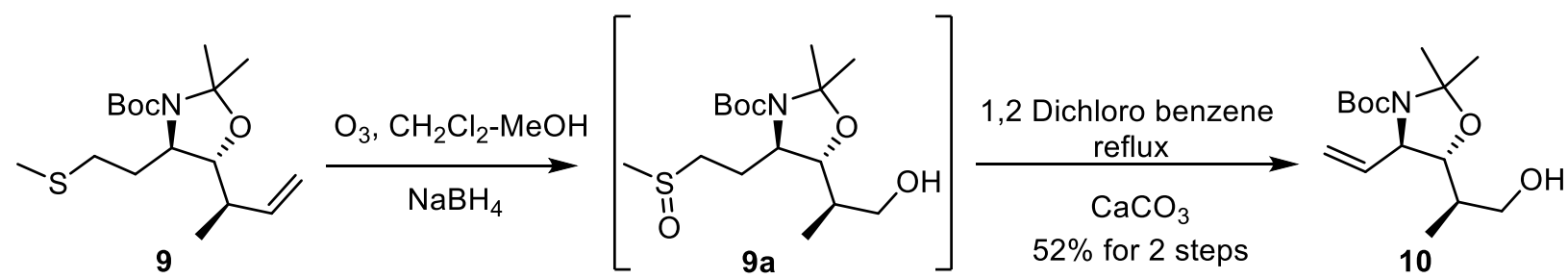

tert-Butyl (4R,5R)-5-((R)-1-hydroxypropan-2-yl)-2,2-dimethyl-4-vinyloxazolidine-3carboxylate (10):

To a solution of compound $\mathbf{1 0}(4.0 \mathrm{~g}, 9.1 \mathrm{mmol})$ in $\mathrm{CH}_{2} \mathrm{Cl}_{2}-\mathrm{MeOH}(1: 1,60 \mathrm{~mL})$ Ozone was bubbled at $-78{ }^{\circ} \mathrm{C}$ until the colour becomes blue, once the blue color appears oxygen was bubbled to remove excess ozone, then $\mathrm{NaBH}_{4}(1.38 \mathrm{~g}, 36.5 \mathrm{mmol})$ was added and reaction mixture was allowed to room temperature and stirred for 8-10 h. Concentrated the reaction mixture, diluted with ethyl acetate $(50 \mathrm{~mL})$ cooled to $0{ }^{\circ} \mathrm{C}$ and quenched with $1 \mathrm{~N} \mathrm{HCl}(5 \mathrm{~mL})$. The organic layer was $\mathrm{H}_{2} \mathrm{O}(10 \mathrm{~mL})$, brine $(10 \mathrm{~mL})$, and evaporated in vacuo to afford alcohol 
compound 9a, here sulfur also got oxidized to sulfoxide. The crude sulfoxide compound was taken in 1,2 dichloro benzene ( $30 \mathrm{~mL}) \mathrm{CaCO}_{3}(3.64 \mathrm{~g}, 36.5 \mathrm{mmol})$ was added and refluxed for 6 h. The crude reaction mixture was purified by column chromatography (silica gel 230-400 mesh $20 \%$ ethyl acetate $\left.-\mathrm{CH}_{2} \mathrm{Cl}_{2}\right)$ to afford $\mathbf{1 0}(1.35 \mathrm{~g}, 52 \%$ for 2 steps $)$ as colorless liquid. $[\alpha]_{\mathrm{D}}{ }^{30}+$ $15.83\left(c\right.$ 0.41, $\left.\mathrm{CHCl}_{3}\right) ;{ }^{1} \mathrm{H}$ NMR $\left(400 \mathrm{MHz}, \mathrm{CDCl}_{3}\right): \delta=5.76-5.57(\mathrm{~m}, 1 \mathrm{H}), 5.14(\mathrm{~d}, J=10.0$ Hz, 2H), 4.06 (brs, 1H), 3.71 (t, $J=6.8 \mathrm{~Hz}, 1 \mathrm{H}), 3.66-3.57$ (m, 2H), 2.56 (brs, 1H), $1.97-1.83$ (m, 1H), 1.57 (s, 3H), 1.46 (s, 3H), 1.39 (brs, 9H), 0.93 (d, $J=6.8 \mathrm{~Hz}, 3 \mathrm{H}) ;{ }^{13} \mathrm{C}$ NMR $(100 \mathrm{MHz}$, $\left.\mathrm{CDCl}_{3}\right): \delta 151.9,138.0,117.2,94.3,83.6,80.1,65.9,63.7,38.3,28.4,26.8,25.9,14.0$; HRMS calculated for $\mathrm{C}_{15} \mathrm{H}_{27} \mathrm{O} 4 \mathrm{NNa}[\mathrm{M}+\mathrm{Na}]^{+}: 308.1832$, found 308.1833 .

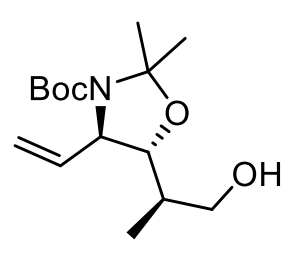

10

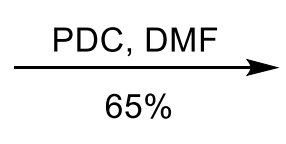

$65 \%$

(S)-2-((4R,5R)-3-(tert-Butoxycarbonyl)-2,2-dimethyl-4-vinyloxazolidin-5-yl)propanoic acid (6): To a stirred solution of compound $10(0.5 \mathrm{~g}, 1.7 \mathrm{mmol})$ in DMF (6 mL), Pyridinium dichromate (PDC) (2.64 g, $7.0 \mathrm{mmol}$ ) was added and stirred at room temperature for $4 \mathrm{~h}$. To the reaction mixture water $(5 \mathrm{~mL})$ was added and extracted with diethyl ether $(20 \mathrm{~mL} \times 2)$, combined the organic layers and washed with brine $(5 \mathrm{~mL})$ concentrated under reduced pressure. Purification by column chromatography (silica gel $100-200$ mesh $30 \%$ ethyl acetate $-\mathrm{CH}_{2} \mathrm{Cl}_{2}$ ) yielded compound $\mathbf{6}(0.34 \mathrm{~g}, 65 \%)$ as a colorless liquid. $[\alpha]_{\mathrm{D}}{ }^{30}+25.09\left(c 0.80, \mathrm{CHCl}_{3}\right) ;{ }^{1} \mathrm{H} \mathrm{NMR}$ $\left(400 \mathrm{MHz}, \mathrm{CDCl}_{3}\right): \delta 5.82-5.67(\mathrm{~m}, 1 \mathrm{H}), 5.20(\mathrm{~d}, J=10.0 \mathrm{~Hz}, 2 \mathrm{H}), 4.28-4.12(\mathrm{~m}, 1 \mathrm{H}), 4.03$ $(\mathrm{dd}, J=5.3,7.7 \mathrm{~Hz}, 1 \mathrm{H}), 2.85-2.70(\mathrm{~m}, 1 \mathrm{H}), 1.62(\mathrm{~s}, 3 \mathrm{H}), 1.53(\mathrm{~s}, 3 \mathrm{H}), 1.45$ (brs, 9H), 1.25 (d, $J=7.1 \mathrm{~Hz}, 3 \mathrm{H}) ;{ }^{13} \mathrm{C} \mathrm{NMR}\left(100 \mathrm{MHz}, \mathrm{CDCl}_{3}\right): \delta 178.9,151.9,137.5,117.4,94.2,81.1,80.2$, 62.7, 43.3, 28.4, 26.7, 26.4, 13.8; HRMS calculated for $\mathrm{C}_{15} \mathrm{H}_{25} \mathrm{O}_{5} \mathrm{NNa}[\mathrm{M}+\mathrm{Na}]^{+}: 322.1625$, found 322.1622 . 
<smiles>Nc1cc(OCc2ccccc2)ccc1I</smiles>

11

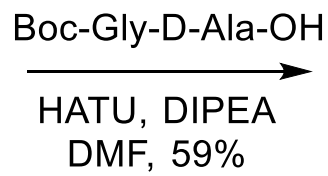

DMF, 59\%<smiles>O=C(CNC(=O)OCc1ccccc1)N[C@@H](F)C(=O)Nc1cc(OCc2ccccc2)ccc1I</smiles>

tert-Butyl (R)-(2-((1-((5-(benzyloxy)-2-iodophenyl)amino)-1-oxopropan-2-yl)amino)-2oxoethyl)carbamate ((5): To a mixture of 5-(benzyloxy)-2-iodoaniline $\mathbf{1 1}^{2}(1.0 \mathrm{~g}, 3.0 \mathrm{mmol})$, Boc-Gly-D-Ala-OH $\left.{ }^{3} 756 \mathrm{mg}, 4.0 \mathrm{mmol}\right)$ in DMF (5 mL). HATU (1.7 g, $\left.4.6 \mathrm{mmol}\right)$, diisopropyl ethylamine $(1.0 \mathrm{~mL}, 6.0 \mathrm{mmol})$ were added at $0{ }^{\circ} \mathrm{C}$ and stirred for $14 \mathrm{~h}$ at $25^{\circ} \mathrm{C}$, the reaction mixture was diluted with ethyl acetate $(30 \mathrm{~mL})$ and washed with $1 \mathrm{~N} \mathrm{HCl}(15 \mathrm{~mL})$ and sat. $\mathrm{NaHCO}_{3}$ solution $\left(15 \mathrm{~mL}\right.$ ) organic layer was separated, dried over $\mathrm{Na}_{2} \mathrm{SO}_{4}$, concentrated under reduced pressure. Purification by column chromatography (silica gel 100-200 mesh 40\% ethyl acetate $\left.-\mathrm{CH}_{2} \mathrm{Cl}_{2}\right)$ yielded compound $5(1.0 \mathrm{~g}, 59 \%)$ as a white color solid. $\mathrm{Mp}=103-104{ }^{\circ} \mathrm{C}$; $[\alpha]_{\mathrm{D}}{ }^{27}+29.51\left(c 1.28, \mathrm{CHCl}_{3}\right) ;$ IR $v_{\max }\left(\right.$ film) $: \mathrm{cm}^{-1} 3362,3020,1695,1584,1514 ;{ }^{1} \mathrm{H}$ NMR (400 MHz, DMSO-d $\left.)_{6}\right): \delta 9.30(\mathrm{~s}, 1 \mathrm{H}), 8.20(\mathrm{~d}, J=6.8 \mathrm{~Hz}, 1 \mathrm{H}), 7.73(\mathrm{~d}, J=8.8 \mathrm{~Hz}, 1 \mathrm{H}), 7.50-7.28$ $(\mathrm{m}, 6 \mathrm{H}), 7.01(\mathrm{t}, J=5.7 \mathrm{~Hz}, 1 \mathrm{H}), 6.72(\mathrm{dd}, J=2.8,8.7 \mathrm{~Hz}, 1 \mathrm{H}), 5.09(\mathrm{~s}, 2 \mathrm{H}), 4.50(\mathrm{~d}, J=6.8 \mathrm{~Hz}$, $1 \mathrm{H}), 3.64(\mathrm{~d}, J=5.9 \mathrm{~Hz}, 2 \mathrm{H}), 1.47-1.27$ (m, 12H) (Boc 9H and Methyl 3H merged); ${ }^{13} \mathrm{C}$ NMR (100 MHz, DMSO-d 6 ): $\delta 171.5,169.8,159.2,156.3,140.1,139.5,137.1,128.9,128.4,128.1$, 114.7, 113.1, 84.3, 78.5, 69.9, 49.2, 43.6, 28.7, 18.5; HRMS calculated for $\mathrm{C}_{23} \mathrm{H}_{28} \mathrm{O}_{5} \mathrm{~N}_{3} \mathrm{INa}$ $[\mathrm{M}+\mathrm{Na}]^{+}:$576.0966, found 576.0963 .
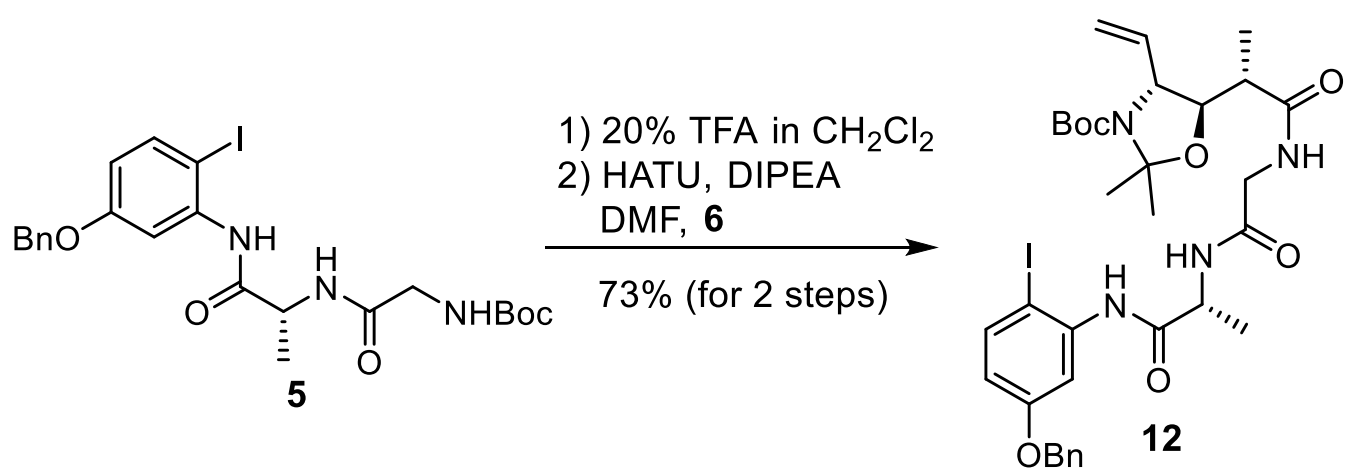

tert-Butyl (4R,5R)-5-((S)-1-((2-(((R)-1-((5-(benzyloxy)-2-iodophenyl)amino)-1-oxopropan-2yl)amino)-2-oxoethyl)amino)-1-oxopropan-2-yl)-2,2-dimethyl-4-vinyloxazolidine-3carboxylate (12): To a solution of $5(665 \mathrm{mg}, 1.2 \mathrm{mmol})$ in $\mathrm{CH}_{2} \mathrm{Cl}_{2}(5 \mathrm{~mL})$, TFA $(1.0 \mathrm{~mL})$ was 
added at $0{ }^{\circ} \mathrm{C}$ and stirred at $25{ }^{\circ} \mathrm{C}$ for $3 \mathrm{~h}$. After completion of the reaction (monitored by TLC), the reaction mixture was concentrated under reduced pressure to afford the amine as TFA salt.

Compound 6 (300 mg, $1.0 \mathrm{mmol}$ ) was taken in dry DMF (5 mL), added above amine salt, then HATU (457 mg, $1.2 \mathrm{mmol})$, DIPEA (0.43 mL, $2.5 \mathrm{mmol})$ were added and the resulting solution was stirred at ambient temperature for $16 \mathrm{~h}$. Reaction mass was diluted with ethyl acetate (50 $\mathrm{mL}$ ) washed with saturated solution of aq $\mathrm{NaHCO}_{3}(5 \mathrm{~mL})$, brine $(5 \mathrm{~mL})$ and then evaporated to dryness. Purification by column chromatography (silica gel 230-400 mesh $4 \%$ methanol $\left.\mathrm{CH}_{2} \mathrm{Cl}_{2}\right)$ yielded compound $12\left(535 \mathrm{mg}, 73 \%\right.$, for 2 steps) as an off white solid. $\mathrm{Mp}=92-94{ }^{\circ} \mathrm{C}$; $[\alpha]_{\mathrm{D}}{ }^{30}+36.09\left(c\right.$ 0.81, $\left.\mathrm{CHCl}_{3}\right) ; \mathrm{IR} v_{\max }\left(\right.$ film) $\mathrm{cm}^{-1} 3020,1687,1584,1518,1381,1217 ;{ }^{1} \mathrm{H}$ NMR (400 MHz, DMSO-d $\left.)_{6}\right): \delta .33$ (s, 1H), $8.15(\mathrm{~d}, J=5.4 \mathrm{~Hz}, 1 \mathrm{H}), 8.04(\mathrm{~d}, J=6.8 \mathrm{~Hz}, 1 \mathrm{H})$, $7.72(\mathrm{~d}, J=8.8 \mathrm{~Hz}, 1 \mathrm{H}), 7.51-7.30(\mathrm{~m}, 5 \mathrm{H}), 7.26$ (brs, $1 \mathrm{H}), 6.80-6.61(\mathrm{~m}, 1 \mathrm{H}), 5.85$ - $5.65(\mathrm{~m}$, 1H), $5.15(\mathrm{~d}, J=15.7 \mathrm{~Hz}, 2 \mathrm{H}), 5.08$ (s, 2H), 4.60 - $4.41(\mathrm{~m}, 1 \mathrm{H}), 4.18$ - $4.00(\mathrm{~m}, 1 \mathrm{H}), 3.97-3.91$ (m, 1H), $3.85(\mathrm{~d}, J=6.4 \mathrm{~Hz}, 1 \mathrm{H}), 3.77-3.62(\mathrm{~m}, 1 \mathrm{H}), 2.69-2.58(\mathrm{~m}, 1 \mathrm{H}), 1.54(\mathrm{~s}, 3 \mathrm{H}), 1.46$ $1.25(\mathrm{~m}, 15 \mathrm{H}), 1.01(\mathrm{~d}, J=6.8 \mathrm{~Hz}, 3 \mathrm{H}) ;{ }^{13} \mathrm{C}$ NMR $\left(100 \mathrm{MHz}, \mathrm{CD}_{3} \mathrm{OD}\right): \delta 176.9,173.3,171.4$, 161.1, 153.6, 140.6, 139.4, 138.2, 129.7, 129.2, 128.8, 118.2, 116.0, 113.8, 95.9, 83.0, 81.6, 71.4, 64.5, 51.0, 46.3, 43.8, 28.8, 27.3, 26.8, 18.2, 14.8; HRMS calculated for $\mathrm{C}_{33} \mathrm{H}_{43} \mathrm{O}_{7} \mathrm{~N}_{4} \mathrm{INa}$ $[\mathrm{M}+\mathrm{Na}]^{+}:$757.2069, found 757.2056.
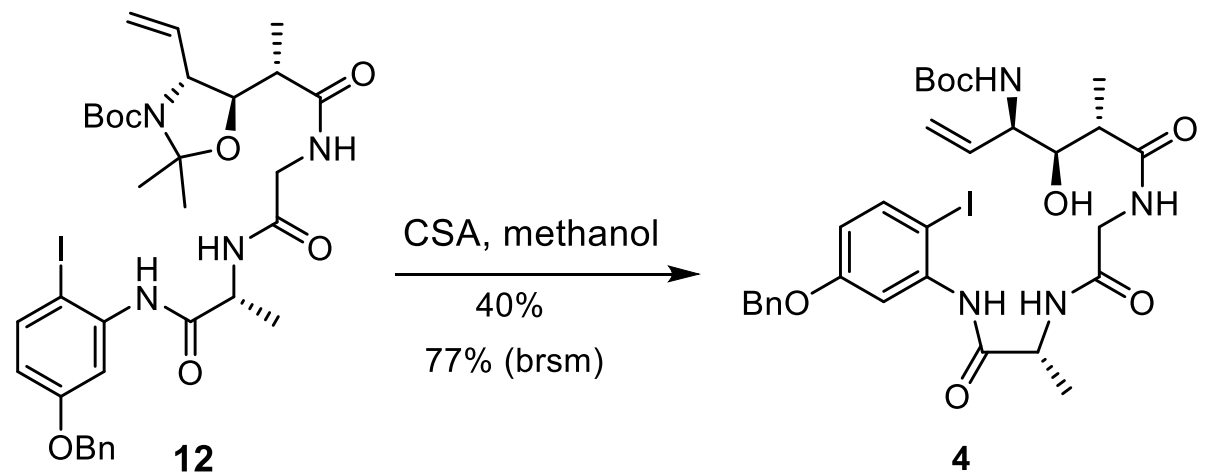

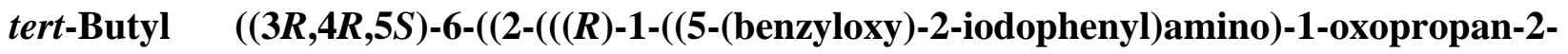
yl)amino)-2-oxoethyl)amino)-4-hydroxy-5-methyl-6-oxohex-1-en-3-yl)carbamate (4) : To a solution of compound 12 (500 mg, $0.6 \mathrm{mmol})$ in methanol (4 mL), Camphorsulphonic acid (31 $\mathrm{mg}, 0.3 \mathrm{mmol}$ ) was added and stirred for $18 \mathrm{~h}$. reaction was monitored by TLC showed only 50\% conversion. Concentrated the reaction mixture diluted with ethyl acetate $(30 \mathrm{~mL})$, washed with saturated solution of aq $\mathrm{NaHCO}_{3}(5 \mathrm{~mL})$, brine $(5 \mathrm{~mL})$ and then evaporated to dryness. 
Purification by column chromatography (silica gel 230-400 mesh 4 - $6 \%$ methanol $-\mathrm{CH}_{2} \mathrm{Cl}_{2}$ ) yielded compound 12 (240 mg) and compound 4 (190 mg, 40\% yield, 77\%, brsm ) as an off white solid. $\mathrm{Mp}=85-86^{\circ} \mathrm{C} ;[\alpha]_{\mathrm{D}}^{27}+27.67$ (c 0.3, $\mathrm{CHCl}_{3}$ ); IR v $v_{\max }(\mathrm{film}): \mathrm{cm}^{-1} 3021,2931$, 1647, 1576; ${ }^{1} \mathrm{H}$ NMR (400MHz, $\left.\mathrm{CD}_{3} \mathrm{OD}\right) \delta 7.71(\mathrm{~d}, J=8.6 \mathrm{~Hz}, 1 \mathrm{H}), 7.51-7.23(\mathrm{~m}, 6 \mathrm{H}), 6.69$ $(\mathrm{dd}, J=2.6,8.7 \mathrm{~Hz}, 1 \mathrm{H}), 5.97-5.83(\mathrm{~m}, 1 \mathrm{H}), 5.29$ - $5.15(\mathrm{~m}, 2 \mathrm{H}), 5.06(\mathrm{~s}, 2 \mathrm{H}), 4.62$ - $4.57(\mathrm{~m}$, 1H) 4.28 (brs, 1H), 4.14 - $4.04(\mathrm{~m}, 1 \mathrm{H}), 3.91-3.81(\mathrm{~m}, 1 \mathrm{H}), 3.76(\mathrm{~d}, J=8.1 \mathrm{~Hz}, 1 \mathrm{H}), 2.66-2.47$ $(\mathrm{m}, 1 \mathrm{H}), 1.51(\mathrm{~d}, J=7.1 \mathrm{~Hz}, 3 \mathrm{H}), 1.46(\mathrm{~s}, 9 \mathrm{H}), 1.17(\mathrm{~d}, J=6.8 \mathrm{~Hz}, 3 \mathrm{H}) ;{ }^{13} \mathrm{C} \mathrm{NMR}(100 \mathrm{MHz}$, $\left.\mathrm{CD}_{3} \mathrm{OD}\right): \delta 177.0,171.9,170.4,159.5,156.6,139.1,137.1,136.7,128.2,127.7,127.3,114.7$, 114.6, 112.6, 82.2, 79.1, 75.1, 69.9, 54.0, 49.7, 43.9, 42.3, 27.4, 16.6, 13.1; HRMS calculated for $\mathrm{C}_{30} \mathrm{H}_{39} \mathrm{O}_{7} \mathrm{~N}_{4} \mathrm{INa}[\mathrm{M}+\mathrm{Na}]^{+}:$717.1756, found 717.1746.
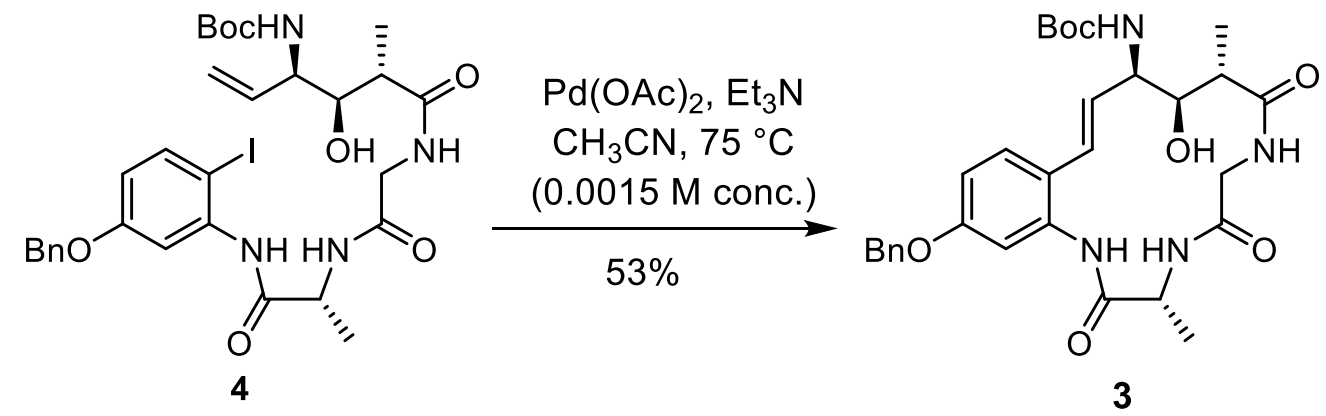

tert-Butyl ((3R,9S,10R,11R,E)-16-(benzyloxy)-10-hydroxy-3,9-dimethyl-2,5,8-trioxo2,3,4,5,6,7,8,9,10,11-decahydro-1H-benzo[ $h][1,4,7]$ triazacyclopentadecin-11-yl)carbamate

(3): To a solution of compound $4(120 \mathrm{mg}, 0.17 \mathrm{mmol})$ in anhydrous acetonitrile $(120 \mathrm{~mL})$, $\mathrm{Pd}(\mathrm{OAc})_{2}(5 \mathrm{~mol} \%)$ and triethylamine $(0.24 \mathrm{~mL}, 1.7 \mathrm{mmol})$ were added and heated at $75{ }^{\circ} \mathrm{C}$ for 12h. The reaction mixture was concentrated in vacuo. Purification by column chromatography (silica gel 230-400 mesh 4\% methanol $-\mathrm{CH}_{2} \mathrm{Cl}_{2}$ ) yielded compound $\mathbf{3}(52 \mathrm{mg}, 53 \%$ ) as an off white solid. $\mathrm{Mp}=130-132{ }^{\circ} \mathrm{C} ;[\alpha]_{\mathrm{D}}{ }^{27}-20.48\left(c 0.81, \mathrm{CHCl}_{3}\right) ;{ }^{1} \mathrm{H}$ NMR $\left(400 \mathrm{MHz}, \mathrm{CD}_{3} \mathrm{OD}\right) \delta$ 7.45 - $7.40(\mathrm{~m}, 2 \mathrm{H}), 7.39$ - $7.33(\mathrm{~m}, 3 \mathrm{H}), 7.31$ (d, J = 8.6 Hz, 1H), 7.28 - 7.25 (m, 1H), 6.90 $6.78(\mathrm{~m}, 1 \mathrm{H}), 6.60(\mathrm{~d}, J=15.7 \mathrm{~Hz}, 1 \mathrm{H}), 5.91$ (dd, $J=8.9,15.8 \mathrm{~Hz}, 1 \mathrm{H}), 5.11-5.03(\mathrm{~m}, 2 \mathrm{H})$, $4.46-4.27(\mathrm{~m}, 2 \mathrm{H}), 4.02(\mathrm{~d}, J=14.4 \mathrm{~Hz}, 1 \mathrm{H}), 3.69(\mathrm{dd}, J=2.2,8.3 \mathrm{~Hz}, 1 \mathrm{H}), 3.62(\mathrm{~d}, J=14.4$ $\mathrm{Hz}, 1 \mathrm{H}), 2.58-2.45(\mathrm{~m}, 1 \mathrm{H}), 1.52(\mathrm{~d}, J=7.3 \mathrm{~Hz}, 3 \mathrm{H}), 1.45(\mathrm{~s}, 9 \mathrm{H}), 1.27(\mathrm{~d}, J=7.3 \mathrm{~Hz}, 3 \mathrm{H}) ;{ }^{13} \mathrm{C}$ NMR (100 MHz, $\left.\mathrm{CD}_{3} \mathrm{OD}\right): \delta 180.9,175.4,174.7,162.3,160.7,143.0,141.0,138.8,132.0$, 131.5, 131.1, 116.9, 115.3, 79.3, 73.6, 62.6, 54.3, 47.6, 46.1, 31.3, 19.3, 19.0; HRMS calculated for $\mathrm{C}_{30} \mathrm{H}_{38} \mathrm{O}_{7} \mathrm{~N}_{4} \mathrm{Na}[\mathrm{M}+\mathrm{Na}]^{+}:$589.2633, found 589.2628. 


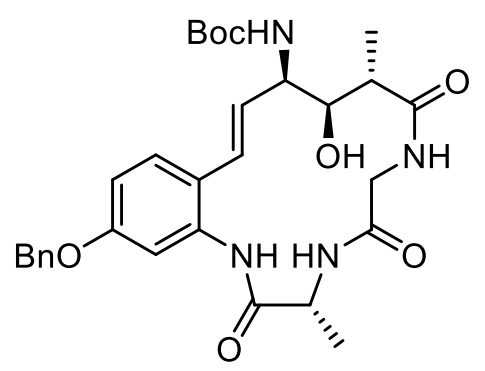

3
1) $20 \%$ TFA in $\mathrm{CH}_{2} \mathrm{Cl}_{2}$

2) HATU, DMF, DIPEA

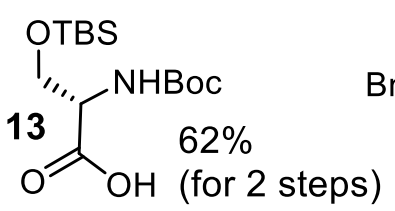

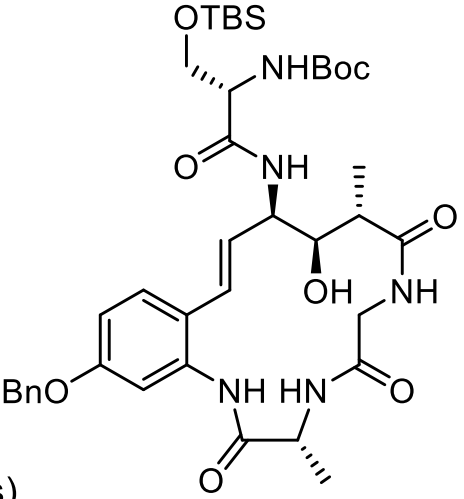

14

tert-Butyl ((S)-1-(((3R,9S,10R,11R,E)-16-(benzyloxy)-10-hydroxy-3,9-dimethyl-2,5,8-trioxo2,3,4,5,6,7,8,9,10,11-decahydro- $1 H$-benzo[ $h][1,4,7]$ triazacyclopentadecin-11-yl)amino)-3-

hydroxy-1-oxopropan-2-yl)carbamate (14): To a solution of compound 3 (45 mg, 0.079 $\mathrm{mmol})$ in $\mathrm{CH}_{2} \mathrm{Cl}_{2}(5 \mathrm{~mL})$ trifluoro acetic acid $(1.0 \mathrm{~mL})$ was added at $0{ }^{\circ} \mathrm{C}$ and the resulting suspension was stirred for $2 \mathrm{~h}$ at the same temperature. Reaction was monitored by TLC, and then concentrated. This residue was dissolved in dry DMF (3 mL), then HATU (60 mg, 0.16 mmol), DIPEA $(41 \mu \mathrm{L}, 0.23 \mathrm{mmol})$ and $N$-(tert-butoxycarbonyl)- $O$-(tert-butyldimethylsilyl)- $L$ serine $\mathbf{1 3}^{4}$ (28 $\left.\mathrm{mg}, 0.087 \mathrm{mmol}\right)$ was added. The resulting solution was stirred at ambient temperature for $16 \mathrm{~h}$. Reaction mass was diluted with ethyl acetate $(15 \mathrm{~mL})$, washed with saturated solution of $\mathrm{NaHCO}_{3}(5 \mathrm{~mL}), \mathrm{H}_{2} \mathrm{O}(5 \mathrm{~mL})$. The organic layer was dried over $\mathrm{Na}_{2} \mathrm{SO}_{4}$ and the crude material obtained after removal of the solvent was purified by column chromatography (silica gel 230-400 mesh 4\% methanol $-\mathrm{CH}_{2} \mathrm{Cl}_{2}$ ) to afford 14 (38 $\mathrm{mg}, 62 \%$ ) as off white solid. $\mathrm{Mp}=87-89{ }^{\circ} \mathrm{C} ;[\alpha]_{\mathrm{D}}{ }^{27}+63.22\left(c 0.12, \mathrm{CHCl}_{3}\right) ;$ IR $v_{\max }($ film $): \mathrm{cm}^{-1} 3023,2403,1523,1595$, 1427; ${ }^{1} \mathrm{H}$ NMR (500 MHz, DMSO-d 6 ): $\delta 8.91(\mathrm{~d}, J=6.6 \mathrm{~Hz}, 1 \mathrm{H}), 8.56$ (brs, $\left.1 \mathrm{H}\right), 8.40$ (d, $J=6.2$ $\mathrm{Hz}, 1 \mathrm{H}), 8.01(\mathrm{~d}, J=7.3 \mathrm{~Hz}, 1 \mathrm{H}), 7.44(\mathrm{~d}, J=7.1 \mathrm{~Hz}, 2 \mathrm{H}), 7.41-7.37$ (m, 3H), $7.35-7.30(\mathrm{~m}$, $1 \mathrm{H}), 7.21(\mathrm{~d}, J=8.5 \mathrm{~Hz}, 1 \mathrm{H}), 6.82(\mathrm{~d}, J=8.7 \mathrm{~Hz}, 1 \mathrm{H}), 6.64-6.43(\mathrm{~m}, 2 \mathrm{H}), 5.86(\mathrm{dd}, J=8.4$, 15.7 Hz, 1H), $5.27(\mathrm{~d}, J=6.9 \mathrm{~Hz}, 1 \mathrm{H}), 5.09$ (s, 2H), $4.63-4.52(\mathrm{~m}, 1 \mathrm{H}), 4.30$ (quin, $J=7.2 \mathrm{~Hz}$, 1H), 4.10 (brs, 2H), 3.79 - 3.72 (m, 1H), 3.66 (brs, 2H), 3.52 - 3.47 (m, 1H), 2.58 - 2.53 (m, 1H), $1.40(\mathrm{~s}, 9 \mathrm{H}), 1.37(\mathrm{~d}, J=7.1 \mathrm{~Hz}, 3 \mathrm{H}), 1.12(\mathrm{~d}, J=7.1 \mathrm{~Hz}, 3 \mathrm{H}), 0.83(\mathrm{~s}, 9 \mathrm{H}), 0.02(\mathrm{~s}, 6 \mathrm{H}) ;{ }^{13} \mathrm{C}$ NMR (125 MHz, DMSO-d 6 ): $\delta$ 175.9, 170.7, 170.0, 169.6, 158.1, 155.6, 137.4, 135.9, 129.1, $128.9,128.3,128.1,127.8,127.0,123.2,112.2,110.8,78.8,75.1,69.7,63.8,56.8,50.2,43.8$, 
41.5, 28.6, 26.2, 18.4, 16.8, -5.0; HRMS calculated for $\mathrm{C}_{39} \mathrm{H}_{58} \mathrm{O}_{9} \mathrm{~N}_{5} \mathrm{Si}[\mathrm{M}+\mathrm{H}]^{+}:$768.3998, found 768.3994.
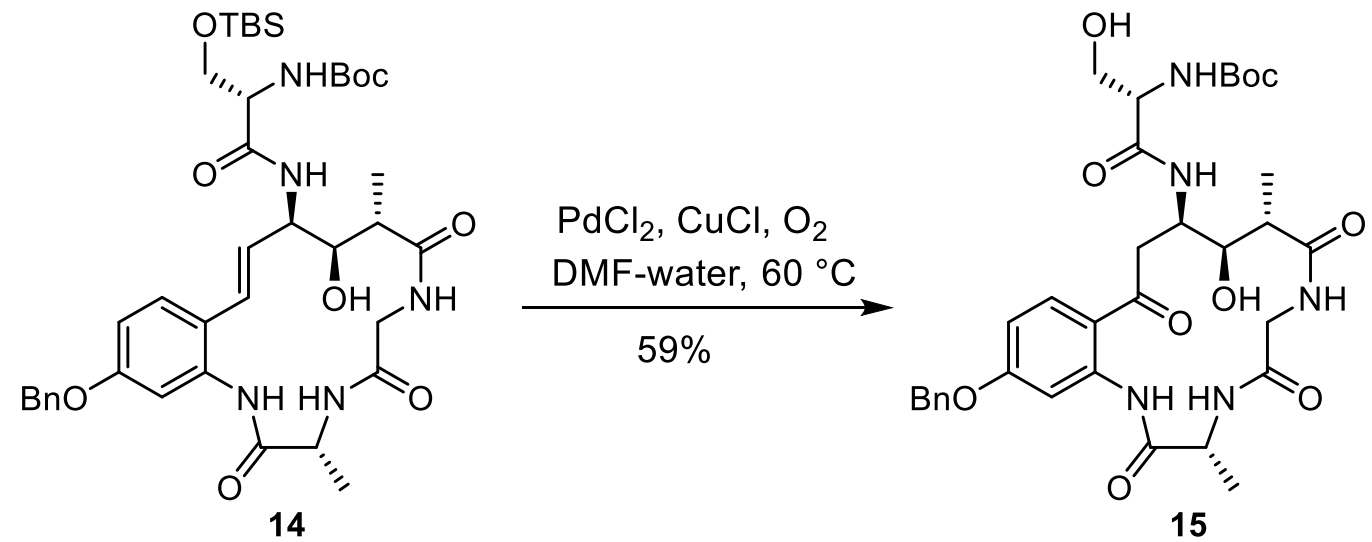

tert-Butyl

$((S)-1-(((3 R, 9 S, 10 R, 11 R)-16-(b e n z y l o x y)-10-h y d r o x y-3,9-d i m e t h y l-2,5,8,13-$ tetraoxo-2,3,4,5,6,7,8,9,10,11,12,13-dodecahydro- $1 H$-benzo $[h][1,4,7]$ triazacyclopentadecin11-yl)amino)-3-hydroxy-1-oxopropan-2-yl)carbamate (15): To a stirred solution of $\mathrm{PdCl}_{2}(10$ $\mathrm{mol} \%), \mathrm{CuCl}(6 \mathrm{mg}, 0.06 \mathrm{mmol})$ in DMF-water ( $3 \mathrm{~mL}, 2: 1)$ compound 14 (50 mg, $0.06 \mathrm{mmol})$ was added and heated at $65{ }^{\circ} \mathrm{C}$ under $\mathrm{O}_{2}$ atmosphere for $8 \mathrm{~h}$. The reaction mixture was diluted with ethyl acetate $(10 \mathrm{~mL})$ and washed water $(5 \mathrm{~mL})$ and brine $(5 \mathrm{~mL})$ organic layer was separated, dried over $\mathrm{Na}_{2} \mathrm{SO}_{4}$, concentrated under reduced pressure. Purification by column chromatography (silica gel 230-400 mesh 6\% methanol - $\mathrm{CH}_{2} \mathrm{Cl}_{2}$ ) yielded compound 15 (26 mg, $59 \%$ ) as a white color solid. $\mathrm{Mp}=242-244{ }^{\circ} \mathrm{C} ;{ }^{1} \mathrm{H}$ NMR $\left(400 \mathrm{MHz}, \mathrm{DMSO}-\mathrm{d}_{6}\right): \delta 12.46(\mathrm{~s}$, $1 \mathrm{H}), 9.10(\mathrm{~d}, J=5.9 \mathrm{~Hz}, 1 \mathrm{H}), 8.32(\mathrm{~d}, J=2.2 \mathrm{~Hz}, 1 \mathrm{H}), 7.92(\mathrm{~d}, J=8.6 \mathrm{~Hz}, 1 \mathrm{H}), 7.73(\mathrm{~d}, J=8.3$ $\mathrm{Hz}, 1 \mathrm{H}), 7.50-7.44(\mathrm{~m}, 2 \mathrm{H}), 7.40(\mathrm{t}, J=7.2 \mathrm{~Hz}, 2 \mathrm{H}), 7.35(\mathrm{~d}, J=7.1 \mathrm{~Hz}, 1 \mathrm{H}), 7.18(\mathrm{~d}, J=7.1$ $\mathrm{Hz}, 1 \mathrm{H}), 6.93(\mathrm{~d}, J=7.6 \mathrm{~Hz}, 1 \mathrm{H}), 6.83(\mathrm{~d}, J=7.8 \mathrm{~Hz}, 1 \mathrm{H}), 5.40(\mathrm{~d}, J=4.6 \mathrm{~Hz}, 1 \mathrm{H}), 5.20$ (s, 2H), 4.88 - $4.74(\mathrm{~m}, 2 \mathrm{H}), 4.36(\mathrm{t}, J=9.3 \mathrm{~Hz}, 1 \mathrm{H}), 4.18$ - $4.06(\mathrm{~m}, 1 \mathrm{H}), 3.93$ - $3.88(\mathrm{~m}, 1 \mathrm{H}), 3.64$ - 3.49 $(\mathrm{m}, 3 \mathrm{H}), 3.32-3.21(\mathrm{~m}, 2 \mathrm{H}), 2.96(\mathrm{~d}, J=17.1 \mathrm{~Hz}, 1 \mathrm{H}), 2.80-2.62(\mathrm{~m}, 1 \mathrm{H}), 1.41(\mathrm{~s}, 9 \mathrm{H}), 1.37$ $(\mathrm{d}, J=4.2 \mathrm{~Hz}, 3 \mathrm{H}), 0.88(\mathrm{~d}, J=6.8 \mathrm{~Hz}, 3 \mathrm{H}) ;{ }^{13} \mathrm{C} \mathrm{NMR}\left(125 \mathrm{MHz}, \mathrm{DMSO}-\mathrm{d}_{6}\right): \delta 201.2,173.0$, $172.8,170.4,169.3,163.5,155.9,142.5,136.6,134.0,129.0,128.9,128.6,128.3,128.1,115.8$, 109.7, 105.7, 78.8, 71.7, 70.0, 61.6, 57.3, 51.2, 45.8, 45.0, 42.8, 42.5, 28.6, 17.4, 9.6; HRMS calculated for $\mathrm{C}_{33} \mathrm{H}_{44} \mathrm{O}_{10} \mathrm{~N}_{5}[\mathrm{M}+\mathrm{H}]^{+}:$670.3083, found 670.3075 . 

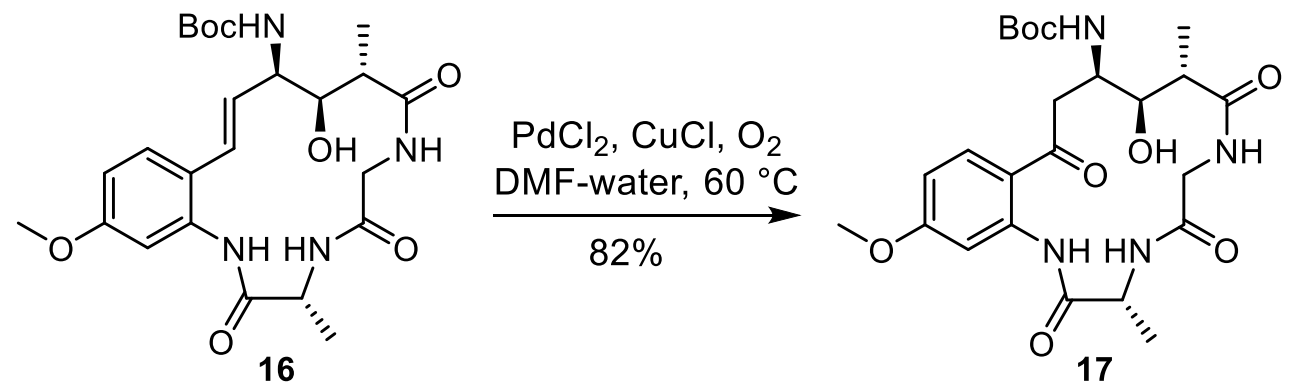

tert-Butyl

((3R,9S,10R,11R)-10-hydroxy-16-methoxy-3,9-dimethyl-2,5,8,13-tetraoxo2,3,4,5,6,7,8,9,10,11,12,13-dodecahydro- $1 H$-benzo[ $h][1,4,7]$ triazacyclopentadecin-11-

yl)carbamate (17) : The compound $17(20 \mathrm{mg}, 82 \%)$ as off white solid was synthesized from compound $16^{5}$ by following similar procedure for the synthesis $15 . \mathrm{Mp}=216-218{ }^{\circ} \mathrm{C} ;{ }^{1} \mathrm{H} \mathrm{NMR}$ (400 MHz, DMSO-d $)_{6}: \delta 12.45(\mathrm{~s}, 1 \mathrm{H}), 9.11(\mathrm{~d}, J=5.6 \mathrm{~Hz}, 1 \mathrm{H}), 8.24(\mathrm{~s}, 1 \mathrm{H}), 8.03(\mathrm{~s}, 1 \mathrm{H}), 7.73$ $(\mathrm{d}, J=9.3 \mathrm{~Hz}, 1 \mathrm{H}), 6.74(\mathrm{dd}, J=2.4,8.8 \mathrm{~Hz}, 1 \mathrm{H}), 5.55(\mathrm{~d}, J=8.8 \mathrm{~Hz}, 1 \mathrm{H}), 5.26(\mathrm{~d}, J=5.4 \mathrm{~Hz}$, 1H), 4.79 (dd, $J=9.0,15.2 \mathrm{~Hz}, 1 \mathrm{H}), 4.24-4.10(\mathrm{~m}, 2 \mathrm{H}), 4.08-4.03(\mathrm{~m}, 1 \mathrm{H}), 3.84$ (s, 3H), 3.74 (brs, 1H), $3.54-3.46$ (m, 1H), 2.97 (d, J=17.4 Hz, 1H), $2.81-2.68$ (m, 1H), 1.39 (s, 12H), 0.93 $(\mathrm{d}, J=6.8 \mathrm{~Hz}, 3 \mathrm{H}) ;{ }^{13} \mathrm{C}$ NMR $\left(100 \mathrm{MHz}, \mathrm{DMSO}-\mathrm{d}_{6}\right): \delta 201.3,172.8,170.4,164.5,154.4,142.6$, 134.2, 115.7, 109.0, 104.7, 78.3, 72.1, 56.1, 51.2, 46.2, 45.1, 43.8, 42.5, 28.7, 17.4, 12.8; HRMS calculated for $\mathrm{C}_{24} \mathrm{H}_{34} \mathrm{O}_{8} \mathrm{~N}_{4} \mathrm{Na}[\mathrm{M}+\mathrm{Na}]^{+}$: 529.2269, found 529.2267.<smiles>COc1ccc(/C=C/[C@@H](N)[C@H](O)[C@H](C)C(=O)NCC(=O)N[C@@H](C)C(=O)OC(C)(C)C)c(NC(=O)[O-])c1</smiles>

16
1) $20 \%$ TFA in $\mathrm{CH}_{2} \mathrm{Cl}_{2}$ 2) HATU, DMF, DIPEA

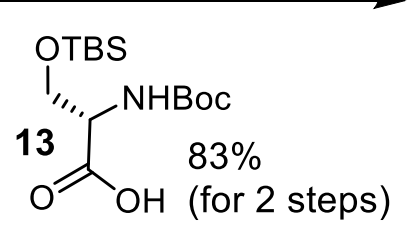

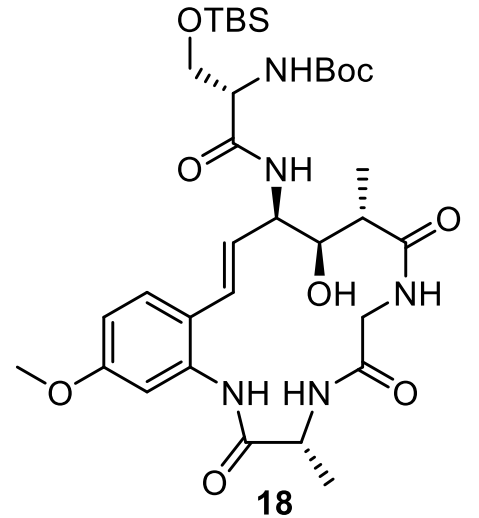

tert-Butyl((S)-3-((tert-butyldimethylsilyl)oxy)-1-(((3R,9S,10R,11R,E)-10-hydroxy-16methoxy-3,9-dimethyl-2,5,8-trioxo-2,3,4,5,6,7,8,9,10,11-decahydro-1 $H$-benzo $[h][1,4,7]$ triazacyclopentadecin-11-yl)amino)-1-oxopropan-2-yl)carbamate (18): To a solution of compound $16^{5}$ (45 mg, $\left.0.091 \mathrm{mmol}\right)$ in $\mathrm{CH}_{2} \mathrm{Cl}_{2}(5 \mathrm{~mL})$ trifluoro acetic acid $(1.0 \mathrm{~mL})$ was added 
at $0{ }^{\circ} \mathrm{C}$ and the resulting suspension was stirred for $2 \mathrm{~h}$ at the same temperature. Reaction was monitored by TLC, and then concentrated. This residue was dissolved in dry DMF (4 mL), then HATU (38 mg, $0.10 \mathrm{mmol})$, DIPEA $(40 \mu \mathrm{L}, 0.22 \mathrm{mmol})$ and $N$-(tert-butoxycarbonyl)-O-(tertbutyldimethylsilyl)- $L$-serine $\mathbf{1 3}^{4}$ (29 $\mathrm{mg}, 0.091 \mathrm{mmol}$ ) was added. The resulting solution was stirred at ambient temperature for $16 \mathrm{~h}$. Reaction mass was diluted with ethyl acetate $(15 \mathrm{~mL})$, washed with saturated solution of $\mathrm{NaHCO}_{3}(5 \mathrm{~mL}), \mathrm{H}_{2} \mathrm{O}(5 \mathrm{~mL})$. The organic layer was dried over $\mathrm{Na}_{2} \mathrm{SO}_{4}$ and the crude material obtained after removal of the solvent was purified by column chromatography (silica gel 230-400 mesh 4\% methanol - $\left.\mathrm{CH}_{2} \mathrm{Cl}_{2}\right)$ to afford $18(52 \mathrm{mg}, 83 \%)$ as off white solid. $\mathrm{Mp}=146-148{ }^{\circ} \mathrm{C} ;[\alpha]_{\mathrm{D}}{ }^{26}+27.10\left(c 0.49, \mathrm{CHCl}_{3}\right) ; \mathrm{IR} v_{\max }\left(\right.$ film) $\mathrm{cm}^{-1} 3023$, 1595, 1217; NMR (400MHz, $\left.\mathrm{CD}_{3} \mathrm{OD}\right) \delta 7.31(\mathrm{~d}, J=8.6 \mathrm{~Hz}, 1 \mathrm{H}), 7.28(\mathrm{~d}, J=2.4 \mathrm{~Hz}, 1 \mathrm{H}), 6.77$ $(\mathrm{dd}, J=2.7,8.6 \mathrm{~Hz}, 1 \mathrm{H}), 6.63(\mathrm{~d}, J=16.1 \mathrm{~Hz}, 1 \mathrm{H}), 6.05(\mathrm{~d}, J=7.6 \mathrm{~Hz}, 1 \mathrm{H}), 4.79$ (t, $J=7.6 \mathrm{~Hz}$, 1H), 4.44 (q, $J=7.1 \mathrm{~Hz}, 1 \mathrm{H}), 4.30-4.21(\mathrm{~m}, 1 \mathrm{H}), 4.09$ (d, $J=15.2 \mathrm{~Hz}, 1 \mathrm{H}), 3.95(\mathrm{~d}, J=6.8 \mathrm{~Hz}$, $1 \mathrm{H}), 3.87(\mathrm{~d}, J=5.9 \mathrm{~Hz}, 2 \mathrm{H}), 3.81(\mathrm{~s}, 3 \mathrm{H}), 3.68(\mathrm{~d}, J=15.2 \mathrm{~Hz}, 1 \mathrm{H}), 2.62$ (d, $J=5.6 \mathrm{~Hz}, 1 \mathrm{H})$, $1.53(\mathrm{~d}, J=7.3 \mathrm{~Hz}, 3 \mathrm{H}), 1.48(\mathrm{~s}, 9 \mathrm{H}), 1.28(\mathrm{~d}, J=7.1 \mathrm{~Hz}, 3 \mathrm{H}), 0.89$ (s, 9H), $0.08(\mathrm{~s}, 6 \mathrm{H}) ;{ }^{13} \mathrm{C}$ NMR (125 MHz, DMSO-d 6 ): 175.9, 170.7, 170.0, 169.6, 159.1, 155.5, 135.9, 129.0, 127.8, 127.0, 122.9, 111.3, 109.7, 78.7, 75.2, 63.7, 56.8, 55.7, 50.1, 43.8, 41.5, 28.6, 26.2, 18.4, 16.8, 5.0; HRMS calculated for $\mathrm{C}_{33} \mathrm{H}_{53} \mathrm{O}_{9} \mathrm{~N}_{5} \mathrm{NaSi}[\mathrm{M}+\mathrm{Na}]^{+}:$714.3505, found 714.3489.

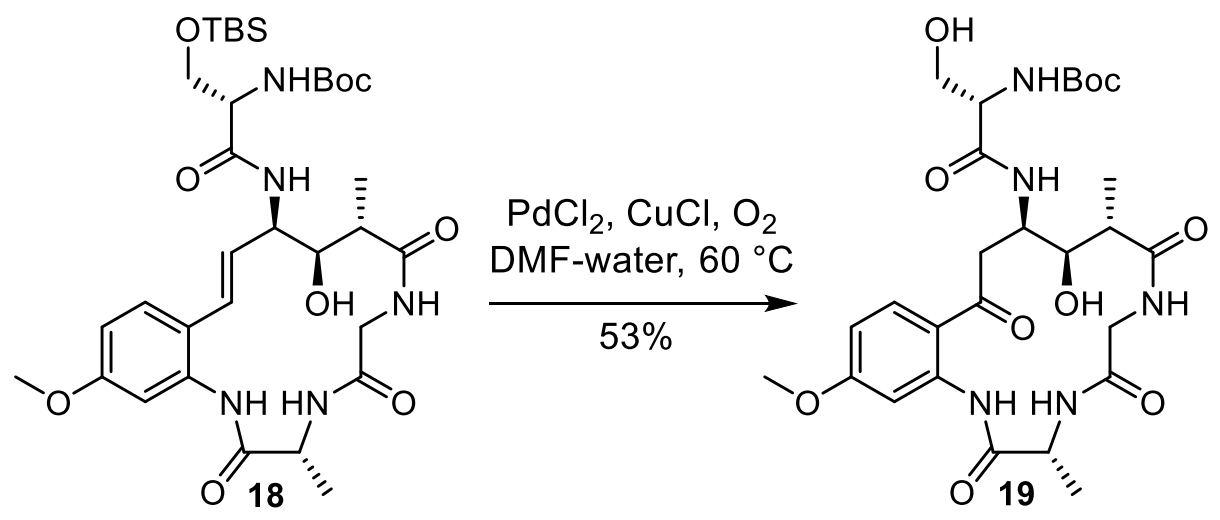

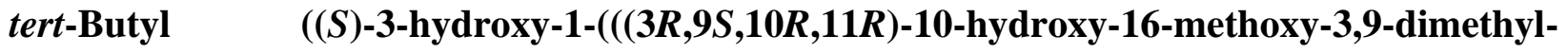
2,5,8,13-tetraoxo-2,3,4,5,6,7,8,9,10,11,12,13-dodecahydro-1 $H$-benzo[h] $[1,4,7]$ triazacyclopentadecin-11-yl)amino)-1-oxopropan-2-yl)carbamate (19): 
The compound 19 (18 $\mathrm{mg}, 53 \%)$ as off white solid was synthesized from compound $\mathbf{1 8}$ by following similar procedure for the synthesis 15. $\mathrm{Mp}=134-136{ }^{\circ} \mathrm{C} ;[\alpha]_{\mathrm{D}}{ }^{26}+0.85(c 0.16$, $\left.\mathrm{CH}_{3} \mathrm{OH}\right) ;{ }^{1} \mathrm{H}$ NMR $\left(400 \mathrm{MHz}, \mathrm{DMSO}-\mathrm{d}_{6}\right): \delta 12.46(\mathrm{~s}, 1 \mathrm{H}), 9.09(\mathrm{~d}, J=5.9 \mathrm{~Hz}, 1 \mathrm{H}), 8.21(\mathrm{~d}, J=$ $2.4 \mathrm{~Hz}, 1 \mathrm{H}), 7.91(\mathrm{~d}, J=8.8 \mathrm{~Hz}, 1 \mathrm{H}), 7.71(\mathrm{~d}, J=8.8 \mathrm{~Hz}, 1 \mathrm{H}), 7.19$ (d, $J=8.1 \mathrm{~Hz}, 1 \mathrm{H}), 6.93$ (d, $J=7.6 \mathrm{~Hz}, 1 \mathrm{H}), 6.75(\mathrm{~d}, J=8.8 \mathrm{~Hz}, 1 \mathrm{H}), 5.45(\mathrm{~d}, J=4.6 \mathrm{~Hz}, 1 \mathrm{H}), 4.87(\mathrm{t}, J=5.4 \mathrm{~Hz}, 1 \mathrm{H}), 4.79$ (dd, $J=9.0,15.4 \mathrm{~Hz}, 1 \mathrm{H}$ ), 4.35 (t, $J=8.4 \mathrm{~Hz}, 1 \mathrm{H}$ ), 4.11 (quin, $J=6.9 \mathrm{~Hz}, 1 \mathrm{H}$ ), $3.94-3.88$ (m, 1H), $3.82(\mathrm{~s}, 3 \mathrm{H}), 3.62-3.52(\mathrm{~m}, 3 \mathrm{H}), 3.32(\mathrm{~s}, 1 \mathrm{H}), 3.16(\mathrm{~d}, J=5.1 \mathrm{~Hz}, 1 \mathrm{H}), 2.95(\mathrm{~d}, J=17.1$ $\mathrm{Hz}, 1 \mathrm{H}), 2.78-2.64(\mathrm{~m}, 1 \mathrm{H}), 1.40(\mathrm{~s}, 9 \mathrm{H}), 1.38(\mathrm{~d}, J=7.8 \mathrm{~Hz}, 3 \mathrm{H}), 0.87(\mathrm{~d}, J=6.8 \mathrm{~Hz}, 3 \mathrm{H}) ;{ }^{13} \mathrm{C}$ NMR (100 MHz, DMSO-d $\left.{ }_{6}\right): \delta 201.1,172.9,172.8,170.4,169.3,164.5,155.9,142.6,134.0$, 115.6, 109.1, 104.7, 78.9, 71.7, 61.7, 57.3, 56.1, 51.2, 45.8, 45.0, 42.8, 42.5, 28.6, 17.4, 9.5; HRMS calculated for $\mathrm{C}_{27} \mathrm{H}_{39} \mathrm{O}_{10} \mathrm{~N}_{5} \mathrm{Na}[\mathrm{M}+\mathrm{Na}]^{+}: 616.2589$, found 616.2589 .

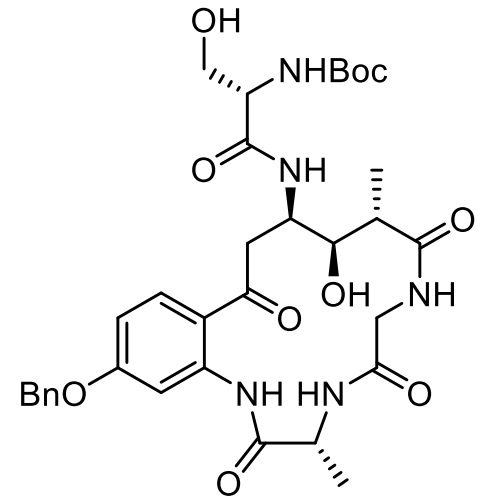

15
1) $10 \% \mathrm{Pd} / \mathrm{C}, \mathrm{H}_{2}$ methanol

2) $10 \%$ TFA in $\mathrm{CH}_{2} \mathrm{Cl}_{2}$ $78 \%$ for 2 steps

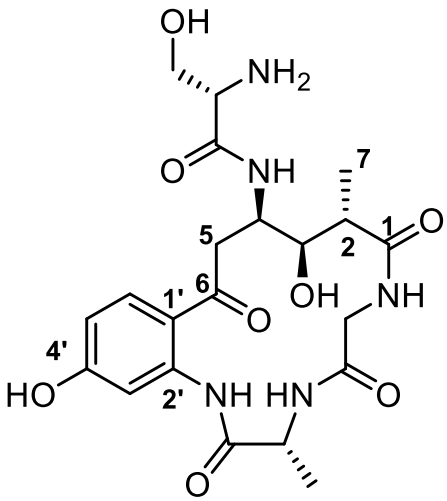

2

tert-Butyl ((S)-1-)((3R,9S,10R,11R)-10,16-dihydroxy-3,9-dimethyl-2,5,8,13-tetraoxo-

\section{2,3,4,5,6,7,8,9,10,11,12,13-dodecahydro- $1 H$-benzo $[h][1,4,7]$ triazacyclopentadecin-11-}

yl)amino)-3-hydroxy-1-oxopropan-2-yl)carbamate (2): To a solution of compound 15 (20 mg, $0.03 \mathrm{mmol})$ in methanol $(3 \mathrm{~mL}), 10 \% \mathrm{Pd} / \mathrm{C}(\sim 5 \mathrm{mg})$ was added and stirred under $\mathrm{H}_{2}$ atmosphere for $2 \mathrm{~h}$. The reaction mixture was then filtered through silica gel column, concentrated to afford phenolic compound. The phenolic compound was dissolved in $\mathrm{CH}_{2} \mathrm{Cl}_{2}(3 \mathrm{~mL})$, TFA $(0.3 \mathrm{~mL})$ was added at $0{ }^{\circ} \mathrm{C}$ and the resulting suspension was stirred for $2 \mathrm{~h}$ at the same temperature. Concentrated the reaction mixture and azeotroped with toluene $(3 \mathrm{~mL} \times 3)$, acetonitrile $(3 \mathrm{~mL} x$ 3) and dried under vacuum to afford compound 2 (11 mg, 78\% for 2 steps) as off white solid. $[\alpha]_{D}^{25}-10.89\left(c 0.34, \mathrm{CH}_{3} \mathrm{OH}\right)$; HRMS calculated for $\mathrm{C}_{29} \mathrm{H}_{30} \mathrm{O}_{8} \mathrm{~N}_{5}[\mathrm{M}+\mathrm{H}]^{+}: 480.2089$, found 480.2085 . 


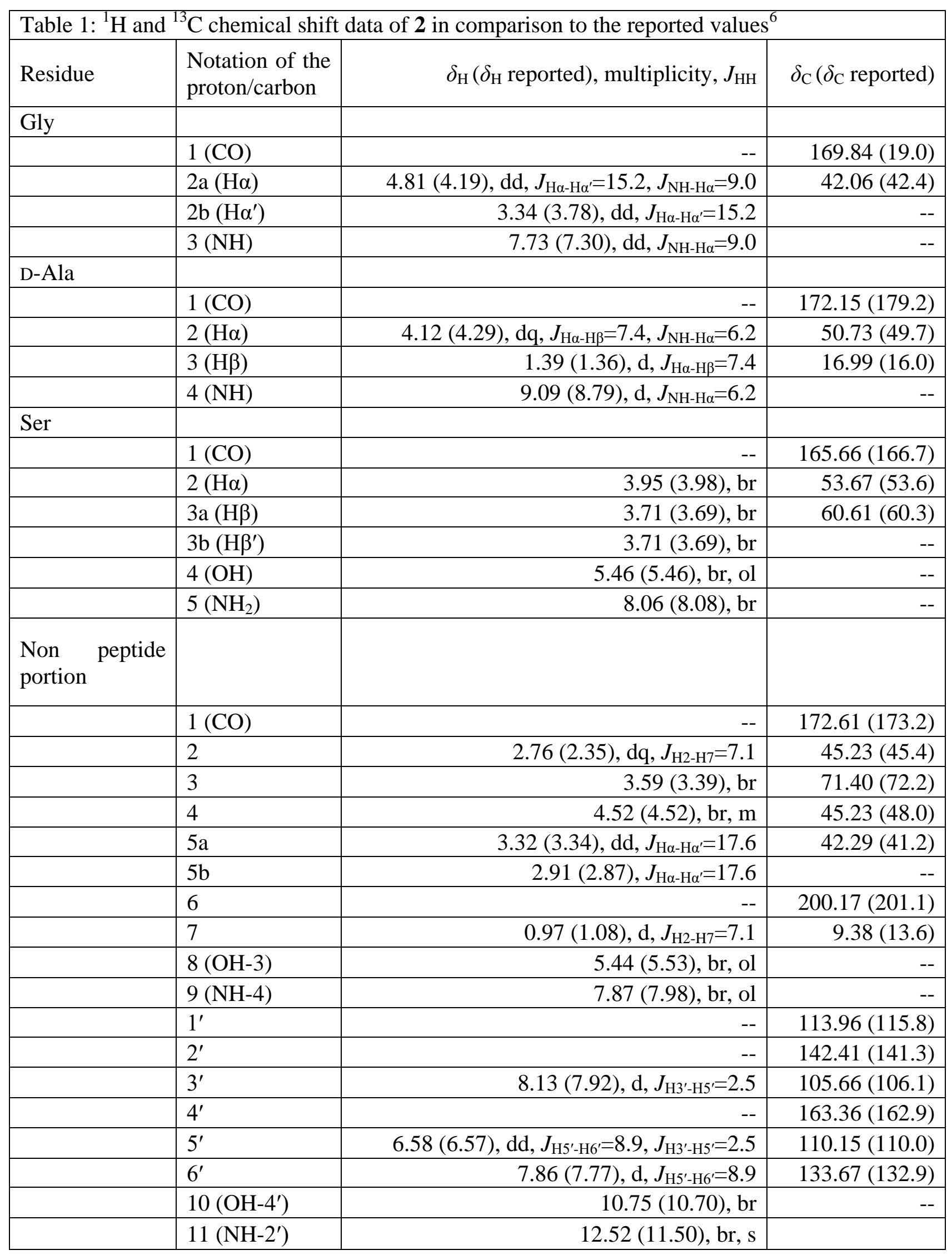


<smiles>C[C@H](NC(=O)CNC(=O)[C@@H](C)[C@H](O)[C@H](N)C=Cc1ccc(OCc2ccccc2)cc1NC(=O)[OH2+])C(=O)OCc1ccccc1</smiles>

3

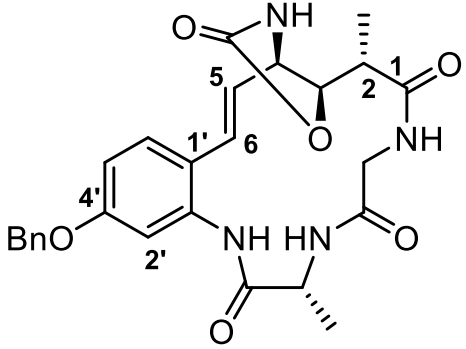

23

(3aR,4S,10R,18aR,E)-14-(Benzyloxy)-4,10-dimethyl-3a,4,6,7,9,10,12,18a-octahydro-2Hbenzo $[\boldsymbol{h}]$ oxazolo $[4,5-1][1,4,7]$ triazacyclopentadecine-2,5,8,11 $(1 \mathrm{H})$-tetraone $\quad(23)$ : $\quad$ To a solution of compound $3(15 \mathrm{mg}, 0.026 \mathrm{mmol})$ in $\mathrm{CH}_{2} \mathrm{Cl}_{2}(5 \mathrm{~mL})$ trifluoro acetic acid (1.0 mL) was added at $0{ }^{\circ} \mathrm{C}$ and the resulting suspension was stirred for $2 \mathrm{~h}$ at the same temperature. Reaction was monitored by TLC, and then concentrated. This residue was dissolved in dry $\mathrm{CH}_{2} \mathrm{Cl}_{2}(4 \mathrm{~mL}$ ), DIPEA ( $9 \mu \mathrm{L}, 0.053 \mathrm{mmol}$ ) followed by triphosgene ( $9 \mathrm{mg}, 0.029 \mathrm{mmol}$, in 1 $\mathrm{mL} \mathrm{CH}_{2} \mathrm{Cl}_{2}$ ) was added at $0{ }^{\circ} \mathrm{C}$ and the resulting suspension was stirred for $2 \mathrm{~h}$. Reaction mixture was diluted with EtOAc $(10 \mathrm{~mL})$ and washed with $1 \mathrm{~N} \mathrm{HCl}(3 \mathrm{~mL})$, brine $(3 \mathrm{~mL})$. Organic layer was separated, dried under vacuum. Purification by column chromatography (silica gel 230-400 mesh 5\% methanol - $\mathrm{CH}_{2} \mathrm{Cl}_{2}$ ) yielded compound $23(8.2 \mathrm{mg}, 63 \%)$ as white solid. $\mathrm{Mp}=236$ $238{ }^{\circ} \mathrm{C} ;[\alpha]_{\mathrm{D}}{ }^{27}+7.11\left(c 0.25, \mathrm{CH}_{3} \mathrm{OH}\right)$; HRMS calculated for $\mathrm{C}_{26} \mathrm{H}_{29} \mathrm{O}_{6} \mathrm{~N}_{4}[\mathrm{M}+\mathrm{H}]^{+}$: 493.2082, found 493.2080.

\begin{tabular}{|c|c|c|c|}
\hline Residue & $\begin{array}{l}\text { Notation of the } \\
\text { proton/carbon }\end{array}$ & $\delta_{\mathrm{H}}$, multiplicity, $J_{\mathrm{H}}$ & $\delta_{\mathrm{C}}$ \\
\hline \multicolumn{4}{|l|}{ Gly } \\
\hline & $1(\mathrm{CO})$ & -- & 172.23 \\
\hline & $2 \mathrm{a}(\mathrm{H} \alpha)$ & $4.27, \mathrm{dd}, J_{\mathrm{H \alpha}-\mathrm{H} \alpha^{\prime}}=15.8, J_{\mathrm{NH}-\mathrm{H} \alpha}=7.7$ & 43.25 \\
\hline & $2 b\left(H \alpha^{\prime}\right)$ & $3.63, \mathrm{dd}, J_{\mathrm{H \alpha}-\mathrm{H} \alpha^{\prime}}=15.8, J_{\mathrm{NH}-\mathrm{H} \alpha^{\prime}}=4.7$ & -- \\
\hline & $3(\mathrm{NH})$ & $8.13, \mathrm{dd}, J_{\mathrm{NH}-\mathrm{H} \alpha}=7.7, J_{\mathrm{NH}-\mathrm{H} \alpha^{\prime}}=4.7$ & \\
\hline \multicolumn{4}{|l|}{ D-Ala } \\
\hline & $1(\mathrm{CO})$ & -- & 171.00 \\
\hline & $2(\mathrm{H \alpha})$ & $4.41, \mathrm{q}, J_{\mathrm{H \alpha}-\mathrm{H} \beta}=7.2, J_{\mathrm{NH}-\mathrm{H \alpha}}=7.2$ & 51.56 \\
\hline
\end{tabular}




\begin{tabular}{|c|c|c|c|}
\hline & $3(\mathrm{H} \beta)$ & $1.43, \mathrm{~d}, J_{\mathrm{H} \alpha-\mathrm{H} \beta}=7.2$ & 15.91 \\
\hline & $4(\mathrm{NH})$ & $8.12, \mathrm{~d}, J_{\mathrm{NH}-\mathrm{H \alpha}}=7.2$ & -- \\
\hline \multicolumn{4}{|l|}{$\begin{array}{l}\text { Non peptide } \\
\text { portion }\end{array}$} \\
\hline & $1(\mathrm{CO})$ & -- & 173.38 \\
\hline & 2 & $3.07, \mathrm{dq}, J_{\mathrm{H} 2-\mathrm{H} 7}=6.9, J_{\mathrm{H} 2-\mathrm{H} 3}=3.6$ & 43.41 \\
\hline & 3 & $4.69, \mathrm{t}, J_{\mathrm{H} 2-\mathrm{H} 3}=3.6, J_{\mathrm{H} 3-\mathrm{H} 4}=3.7$ & 82.48 \\
\hline & 4 & $4.73, \mathrm{ddd}, J_{\mathrm{H} 4-\mathrm{H} 5}=7.0, J_{\mathrm{H} 3-\mathrm{H} 4}=3.7, J_{\mathrm{H} 4-\mathrm{H} 8}=1.8$ & 54.03 \\
\hline & 5 & $6.02, \mathrm{dd}, J_{\mathrm{H} 5-\mathrm{H} 6}=15.8, J_{\mathrm{H} 4-\mathrm{H} 5}=7.0$ & 134.05 \\
\hline & 6 & $6.48, \mathrm{~d}, J_{\mathrm{H} 5-\mathrm{H} 6}=15.8$ & 127.72 \\
\hline & 7 & $1.18, \mathrm{~d}, J_{\mathrm{H} 2-\mathrm{H} 7}=6.9$ & 8.71 \\
\hline & 8 (NH-4) & $7.02, \mathrm{br}$ & -- \\
\hline & $1^{\prime}$ & -- & 122.86 \\
\hline & $2^{\prime}$ & -- & 136.92 \\
\hline & $3^{\prime}$ & $7.66, \mathrm{~d}, J_{\mathrm{H}^{\prime}-\mathrm{H} 5^{\prime}}=2.6$ & 110.04 \\
\hline & $4^{\prime}$ & -- & 159.44 \\
\hline & $5^{\prime}$ & $6.77, \mathrm{dd}, J_{\mathrm{H} 5^{\prime}-\mathrm{H} 6^{\prime}}=8.6, J_{\mathrm{H}^{\prime}-\mathrm{H} 5^{\prime}}=2.6$ & 112.11 \\
\hline & $6^{\prime}$ & $7.28, \mathrm{~d}, J_{\mathrm{H} 5^{\prime}-\mathrm{H} 6^{\prime}}=8.6$ & 128.70 \\
\hline & $9\left(\mathrm{NH}-2^{\prime}\right)$ & $8.86, \mathrm{~s}$ & -- \\
\hline \multicolumn{4}{|l|}{ OBn-6' } \\
\hline & $1^{\prime \prime}$ & -- & 138.05 \\
\hline & $2 "$ & $7.47, \mathrm{dd},, J_{\mathrm{H} 2^{\prime \prime}-\mathrm{H} 3^{\prime \prime}}=7.2, J_{\mathrm{H} 2^{\prime \prime}-\mathrm{H} 4^{\prime \prime}}=1.7$ & 128.28 \\
\hline & $3 "$ & $7.38, \mathrm{t}, J_{\mathrm{H} 2^{\prime \prime}-\mathrm{H} 3^{\prime \prime}}=7.2, J_{\mathrm{H} 3^{\prime \prime}-\mathrm{H} 4^{\prime \prime}}=7.2$ & 129.12 \\
\hline & $4 "$ & $7.31, \mathrm{tt}, J_{\mathrm{H} 3^{\prime \prime}-\mathrm{H} 4^{\prime \prime}}=7.2, J_{\mathrm{H} 2^{\prime \prime}-\mathrm{H} 4^{\prime \prime}}=1.7$ & 128.28 \\
\hline & $1\left(\mathrm{CH}_{2}-1^{\prime \prime}\right)$ & $5.09, \mathrm{~s}$ & 70.27 \\
\hline
\end{tabular}


The observed ROE pattern for 23 (Figure 1) H2-H3 (very strong), H6-H3 (strong), H6-H4 (strong), H5-H3 (strong), H5-H9 (medium), H7-H3 (strong), Gly-NH-H2 (strong), Gly-NH-H3 (strong), Gly-NH-H4 (weak), Gly-NH-H7 (very weak), H4-H7 (very strong) and H9-H7 (very weak) well support the relative stereochemistry. Further, the measured ${ }^{3} J_{\mathrm{H}}$ couplings ${ }^{3} J_{\mathrm{H} 2-\mathrm{H} 3}=3.6$ $\mathrm{Hz}$ and ${ }^{3} \mathrm{~J}_{\mathrm{H} 3-\mathrm{H} 4}=3.7 \mathrm{~Hz}$ with respective dihedral angles of $\sim 50^{\circ}$ and $\sim 130^{\circ}$ (as calculated based on Karplus equation $)^{[7]}$ for $\mathrm{H} 2-\mathrm{C} 2-\mathrm{C} 3-\mathrm{H} 3$ and $\mathrm{H} 3-\mathrm{C} 3-\mathrm{C} 4-\mathrm{H} 4$, respectively, were also fulfilled in the probable conformation for $\mathbf{2 3}$ with which the above ROEs were satisfied.

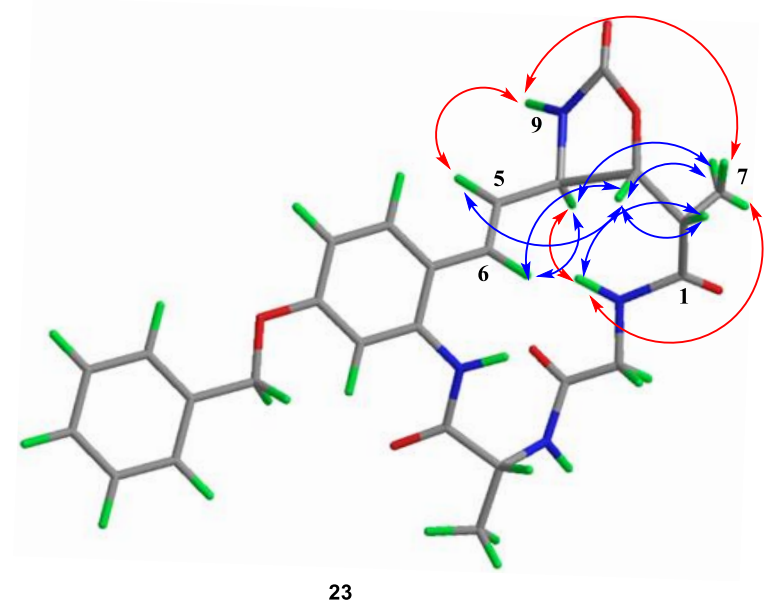

Figure1: The key ROE pattern (shown in curved double headed arrows - very strong and strong ROEs in blue; medium to weak ROEs in red) observed for $\mathbf{2 3}$ in support of the relative stereochemical configuration at positions 2, 3 and 4 . The core structure is drawn in chem.3D for an appropriate representation of the ROE pattern

X-ray Crystal Structure Details of compound 23: Single crystals of compound 23, obtained from Acetone-d6. X-ray intensity data were collected on a Bruker SMART APEX II CCD diffractometer with graphite-monochromatized (Mo $\mathrm{K} \alpha=0.71073 \AA$ ) radiation. The $\mathrm{X}$-ray generator was operated at $50 \mathrm{kV}$ and $30 \mathrm{~mA}$. A preliminary set of cell constants and an orientation matrix were calculated from three sets of 36 frames. Data were collected with $\omega$ scan width of $0.5^{\circ}$ at different settings of $\varphi$ and $2 \theta$ with a frame time of 40 secs keeping the sampleto-detector distance fixed at $5.00 \mathrm{~cm}$. The X-ray data collection was monitored by APEX2 program (Bruker, 2006). All the data were corrected for Lorentzian, polarization and absorption effects using SAINT and SADABS programs (Bruker, 2006). SHELX-97 was used for structure solution and full matrix least-squares refinement on $F^{2}$. All the hydrogen atoms were placed in 
geometrically idealized position and constrained to ride on their parent atoms. An ORTEP III view of both compounds were drawn with $30 \%$ probability displacement ellipsoids and $\mathrm{H}$ atoms are shown as small spheres of arbitrary radii. In the crystal structure benzyl group showed orientational disorder over two positions having occupancy 0.7 and 0.3 . The anisotropic parameters of the benzyl group atoms were restraints using DELU, SIMU and ISOR commands integrated in SHELXTL package.

Crystallographic data for $23\left(\mathrm{C}_{26} \mathrm{H}_{28} \mathrm{~N}_{4} \mathrm{O}_{6}\right): M=492.52$, Crystal dimensions 0.46 x 0.20 x 0.11 $\mathrm{mm}^{3}$, monoclinic, space group $C 2, a=21.150(10), b=4.978(3), c=24.550(11) \AA, \beta=$ 104.86(3) $)^{\circ}, V=2498(2) \AA^{3}, Z=4, \rho_{\text {calcd }}=1.310 \mathrm{gcm}^{-3}, \mu\left(\mathrm{Mo}-\mathrm{K}_{\alpha}\right)=0.094 \mathrm{~mm}^{-1}, F(000)=$ $1040,2 \theta_{\max }=50.00^{\circ}, T=296(2) \mathrm{K}, 14707$ reflections collected, 4389 unique reflections $\left(R_{\mathrm{int}}=0.1283\right), 2757$ observed $(I>2 \sigma(I))$ reflections, multi-scan absorption correction, $T_{\min }=$ $0.958, T_{\max }=0.990,379$ refined parameters, No. of restraints 199, $S=1.070, R 1=0.0834, w R 2$ $=0.1716$ (all data $R=0.1345, w R 2=0.1956$ ), maximum and minimum residual electron densities; $\Delta \rho_{\max }=0.30, \Delta \rho_{\min }=-0.23\left(\mathrm{e}^{-3}\right)$. Crystallographic data for compound $\mathbf{2 3}$ deposited with the Cambridge Crystallographic Data Centre as supplementary publication no.CCDC

1427296.

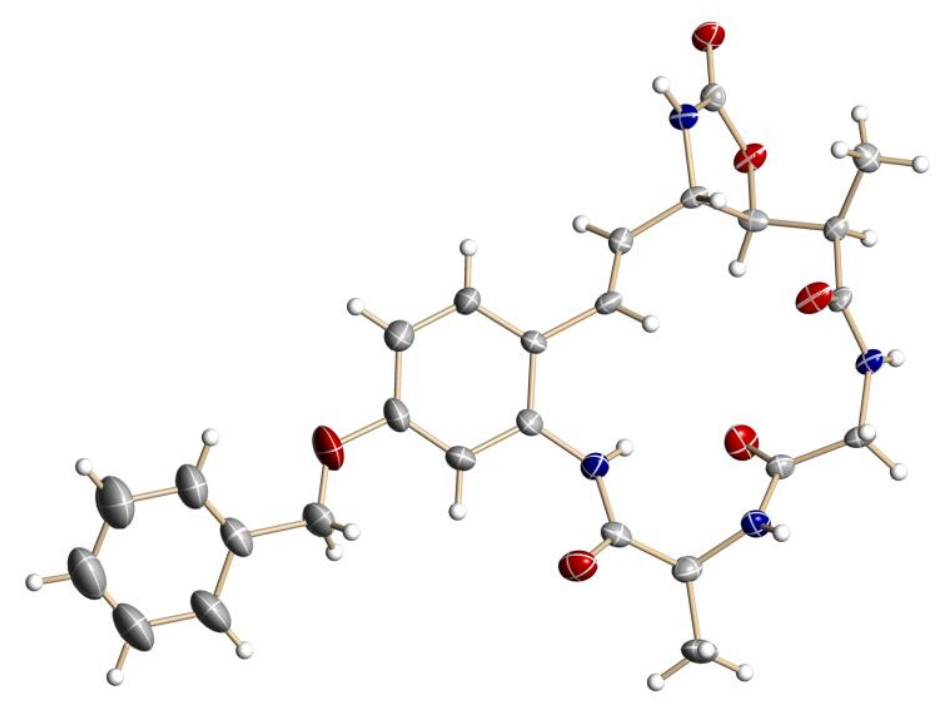

Figure 1: ORTEP diagram of compound 23. 


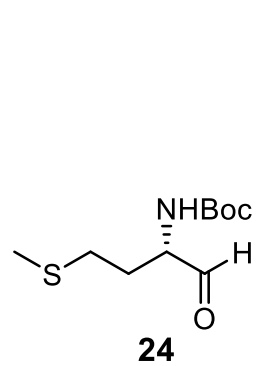

24
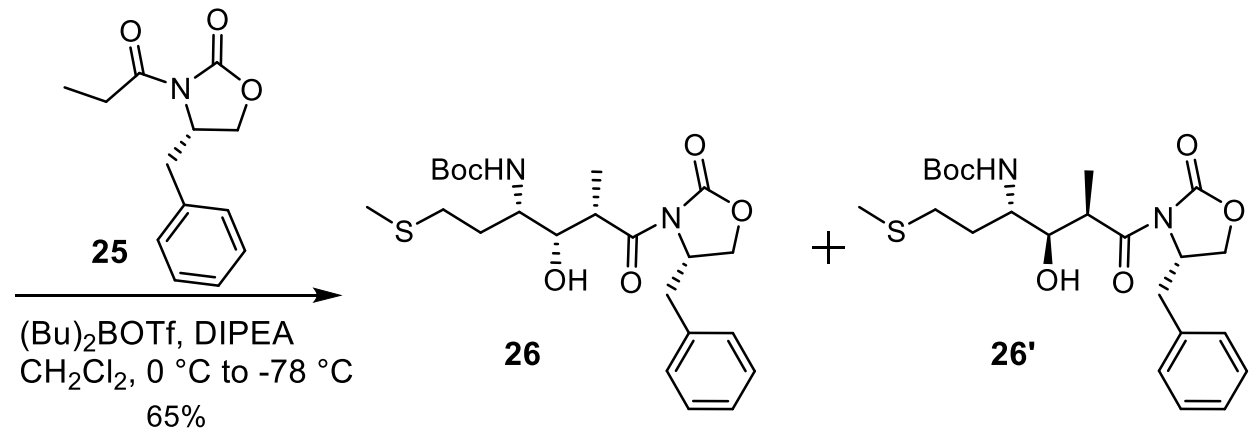

tert-Butyl

((3S,4S,5S)-6-((S)-4-benzyl-2-oxooxazolidin-3-yl)-4-hydroxy-5-methyl-1(methylthio)-6-oxohexan-3-yl)carbamate (26) ${ }^{8}$ and tert-butyl ((3S,4R,5R)-6-((S)-4-benzyl-2oxooxazolidin-3-yl)-4-hydroxy-5-methyl-1-(methylthio)-6-oxohexan-3-yl)carbamate $\quad\left(266^{\prime}\right)$ : To a stirred solution of oxazolidinone $25^{9}(7.0 \mathrm{~g}, 30.0 \mathrm{mmol})$ in $\mathrm{CH}_{2} \mathrm{Cl}_{2}(70 \mathrm{~mL})$ was added $\mathrm{Bu}_{2}$ BOTf $(33 \mathrm{~mL}, 33.0 \mathrm{mmol})$ and DIPEA $(6.2 \mathrm{~mL}, 36.0 \mathrm{mmol})$ at $0{ }^{\circ} \mathrm{C}$. After stirring for $1 \mathrm{~h}$, the solution was cooled to $-78{ }^{\circ} \mathrm{C}$ and maintained at $-78{ }^{\circ} \mathrm{C}$ for $30 \mathrm{~min}$. A solution containing aldehyde $24(7.7 \mathrm{~g}, 33.0 \mathrm{mmol})$ in $\mathrm{CH}_{2} \mathrm{Cl}_{2}(30 \mathrm{~mL})$ was added dropwise and the solution was allowed to warm slowly to room temperature and stirred for overnight. The reaction was quenched by the addition of aqueous ammonium chloride solution $(10 \mathrm{~mL})$ at $0{ }^{\circ} \mathrm{C}$ and then $\mathrm{H}_{2} \mathrm{O}$ $(30 \mathrm{~mL})$ was added to the reaction mixture. The mixture was extracted with $\mathrm{CH}_{2} \mathrm{Cl}_{2}(3 \times 50 \mathrm{~mL})$. The combined organic extracts were dried over anhydrous sodium sulphate, filtered, and concentrated in vacuo. The organic residue was purified by column chromatography (silica gel 230-400 mesh 15\% ethyl acetate - pet ether) to afford 26 and 26' with 7:3 Diastereomeric ratio.

Data for compound 26: $[\alpha]_{\mathrm{D}}{ }^{26}+44.48\left(c 0.95, \mathrm{CH}_{3} \mathrm{OH}\right)$; IR $v_{\max }\left(\right.$ film) $: \mathrm{cm}^{-1} 3439,3019,2980$, 1781, 1693, 1502; ${ }^{1} \mathrm{H} \mathrm{NMR}\left(400 \mathrm{MHz}, \mathrm{CDCl}_{3}\right): \delta 7.35-7.21(\mathrm{~m}, 5 \mathrm{H}), 4.78(\mathrm{t}, J=10.0 \mathrm{~Hz}, 2 \mathrm{H})$, $4.32(\mathrm{t}, J=8.1 \mathrm{~Hz}, 1 \mathrm{H}), 4.14(\mathrm{~d}, J=8.8 \mathrm{~Hz}, 1 \mathrm{H}), 3.89$ (brs, $1 \mathrm{H}), 3.81$ - 3.71 (m, 2H), 3.25 - 3.22 $(\mathrm{m}, 1 \mathrm{H}), 2.82(\mathrm{dd}, J=9.5,13.0 \mathrm{~Hz}, 1 \mathrm{H}), 2.60-2.50(\mathrm{~m}, 2 \mathrm{H}), 2.47-2.46(\mathrm{~m}, 1 \mathrm{H}), 2.12(\mathrm{~s}, 3 \mathrm{H})$, $1.86(\mathrm{q}, J=7.5 \mathrm{~Hz}, 2 \mathrm{H}), 1.40(\mathrm{~s}, 9 \mathrm{H}), 1.38(\mathrm{~d}, 6.4 \mathrm{~Hz}, 3 \mathrm{H}) ;{ }^{13} \mathrm{C} \mathrm{NMR}\left(100 \mathrm{MHz}, \mathrm{CDCl}_{3}\right): \delta$ 175.8, 156.3, 153.2, 135.4, 129.4, 128.9, 127.3, 79.6, 74.7, 66.4, 55.3, 51.8, 40.9, 38.1, 32.8, 30.8, 28.3, 15.6, 14.8; HRMS calculated for $\mathrm{C}_{23} \mathrm{H}_{34} \mathrm{O}_{6} \mathrm{~N}_{2} \mathrm{NaS}[\mathrm{M}+\mathrm{Na}]^{+}$: 489.2030, found 489.2027.

Data for compound 26': $[\alpha]_{\mathrm{D}}^{26}+66.87\left(c 0.59, \mathrm{CH}_{3} \mathrm{OH}\right)$; IR $v_{\max }\left(\right.$ film) $\mathrm{cm}^{-1} 3443,3014,2995$, 1788, 1685, 1508; ${ }^{1} \mathrm{H}$ NMR (400 MHz, $\left.\mathrm{CDCl}_{3}\right): \delta 7.33$ - $7.17(\mathrm{~m}, 5 \mathrm{H}), 4.67$ - 4.65 (m, 2H), 4.23 - $4.15(\mathrm{~m}, 2 \mathrm{H}), 3.93(\mathrm{~s}, 1 \mathrm{H}), 3.84-3.82(\mathrm{~m}, 1 \mathrm{H}), 3.78-3.73(\mathrm{~m}, 1 \mathrm{H}), 3.23(\mathrm{~d}, J=13.3 \mathrm{~Hz}, 1 \mathrm{H})$, 
$2.78-2.73(\mathrm{~m}, 1 \mathrm{H}), 2.57-2.50(\mathrm{~m}, 2 \mathrm{H}), 2.10-2.08(\mathrm{~m}, 1 \mathrm{H}), 2.07(\mathrm{~s}, 3 \mathrm{H}), 1.65-1.63(\mathrm{~m}, 1 \mathrm{H})$, $1.41(\mathrm{~s}, 9 \mathrm{H}), 1.30(\mathrm{~d}, J=6.4 \mathrm{~Hz}, 3 \mathrm{H}) ;{ }^{13} \mathrm{C} \mathrm{NMR}\left(100 \mathrm{MHz}, \mathrm{CDCl}_{3}\right): \delta 177.0,155.7,152.8$, 135.0, 129.4, 128.9, 127.4, 79.7, 74.0, 66.2, 55.1, 51.8, 39.6, 37.7, 30.9, 30.6, 28.3, 15.6, 11.1; HRMS calculated for $\mathrm{C}_{23} \mathrm{H}_{34} \mathrm{O}_{6} \mathrm{~N}_{2} \mathrm{NaS}[\mathrm{M}+\mathrm{Na}]^{+}$: 489.2030, found 489.2027.

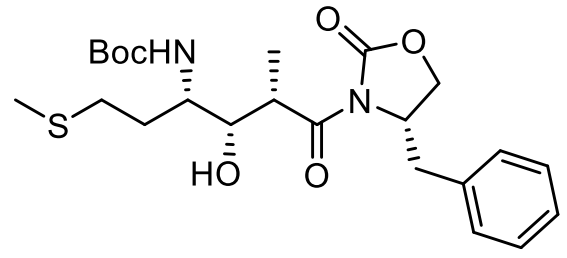

26

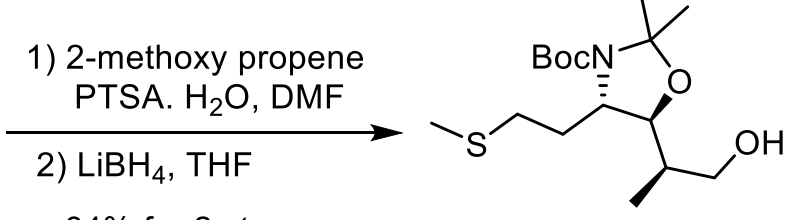

$64 \%$ for 2 steps

tert-Butyl （4R,5R)-5-((S)-1-((S)-4-benzyl-2-oxooxazolidin-3-yl)-1-oxopropan-2-yl)-2,2dimethyl-4-(2-(methylthio)ethyl)oxazolidine-3-carboxylate (27): To a stirred solution of compound 26 (6.0 g, $12.87 \mathrm{mmol})$, and 2-methoxypropene (2.78 mL, $38.62 \mathrm{mmol})$ in dry DMF (20 mL) PTSA. $\mathrm{H}_{2} \mathrm{O}(442 \mathrm{mg}, 2.57 \mathrm{mmol})$ was added at $0{ }^{\circ} \mathrm{C}$ under argon atmosphere. The resulting solution was stirred at room temperature for $4 \mathrm{~h}$. The reaction was then diluted with $\mathrm{H}_{2} \mathrm{O}(5 \mathrm{~mL})$ and extracted with EtOAc $(2 \times 50 \mathrm{~mL})$. The combined organic layers were washed with cold saturated $\mathrm{NaHCO}_{3}$ solution $(15 \mathrm{~mL}), \mathrm{H}_{2} \mathrm{O}(10 \mathrm{~mL})$, brine $(10 \mathrm{~mL})$, and evaporated in vacuo. The crude acetonide protected $(5.5 \mathrm{~g}, 10.8 \mathrm{mmol})$ compound was taken in dry THF (30 $\mathrm{mL})$ at $0{ }^{\circ} \mathrm{C}, \mathrm{LiBH}_{4}(0.70 \mathrm{~g}, 32.4 \mathrm{mmol})$ was added portion wise under argon atmosphere stirred at room temperature for $5 \mathrm{~h}$. Excess $\mathrm{LiBH}_{4}$ was decomposed with $1 \mathrm{~N} \mathrm{HCl}$ and extracted with EtOAc $(2 \times 50 \mathrm{~mL})$. The combined organic layers were washed with cold saturated brine (10 $\mathrm{mL}$ ), dried over $\mathrm{Na}_{2} \mathrm{SO}_{4}$ and evaporated in vacuo. The organic residue was purified by column chromatography (silica gel 230-400 mesh $15 \%$ ethyl acetate - pet ether) to afford 27 as a oil (2.75 g, 64\% yield over two steps); $[\alpha]_{\mathrm{D}}{ }^{26}+15.862$ (c $\left.0.51, \mathrm{CH}_{3} \mathrm{OH}\right) ; \mathrm{IR} v_{\max }\left(\right.$ film): $\mathrm{cm}^{-1} 3384$, 3020, 2982, 1689, 1525, 1390; ${ }^{1} \mathrm{H}$ NMR (400 MHz, $\left.\mathrm{CDCl}_{3}\right): \delta 3.91-3.89(\mathrm{~m}, 2 \mathrm{H}), 3.62(\mathrm{dd}, J=$ 1.6, $5.3 \mathrm{~Hz}, 2 \mathrm{H}), 2.52$ - $2.45(\mathrm{~m}, 2 \mathrm{H}), 2.11(\mathrm{~s}, 3 \mathrm{H}), 1.98$ - $1.81(\mathrm{~m}, 2 \mathrm{H}), 1.47(\mathrm{~s}, 15 \mathrm{H}), 1.01(\mathrm{~d}, J=6.9 \mathrm{~Hz}$, $3 \mathrm{H}) ;{ }^{13} \mathrm{C}$ NMR $\left(100 \mathrm{MHz}, \mathrm{CDCl}_{3}\right): \delta$ 152.0, 94.0, 81.2, 80.1, 66.0, 59.0, 38.4, 31.9, 30.4, 28.5, 27.5, 27.3, 15.6, 11.7; HRMS calculated for $\mathrm{C}_{16} \mathrm{H}_{31} \mathrm{O}_{4} \mathrm{NNaS}[\mathrm{M}+\mathrm{Na}]^{+}$: 356.1866, found 356.1861. 


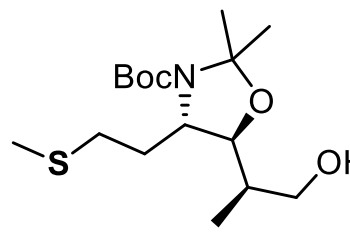

27

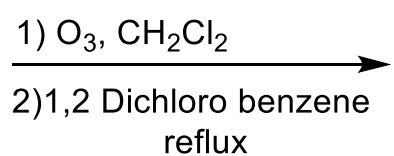

$31 \%$ for 2 steps

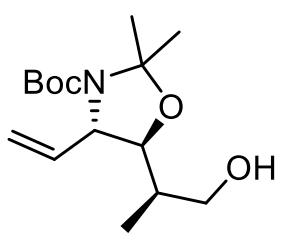

$27 a$

tert-Butyl

(4S,5S)-5-((R)-1-hydroxypropan-2-yl)-2,2-dimethyl-4-vinyloxazolidine-3-

carboxylate (27a): To a solution of compound $27(3.0 \mathrm{~g}, 9.0 \mathrm{mmol})$ in $\mathrm{CH}_{2} \mathrm{Cl}_{2}(60 \mathrm{~mL})$ Ozone was bubbled at $-78{ }^{\circ} \mathrm{C}$ until the colour becomes blue, once the blue color appears oxygen was bubbled to remove excess ozone, then reaction mixture was allowed to warm to room temperature and stirred for $1 \mathrm{~h}$. Concentrated the reaction mixture, here sulfur got oxidized to sulfoxide. The crude sulfoxide compound was taken in 1,2 dichloro benzene $(30 \mathrm{~mL}), \mathrm{CaCO}_{3}$ $(2.7 \mathrm{~g}, 27.0 \mathrm{mmol})$ was added and refluxed for $6 \mathrm{~h}$. The crude reaction mixture was purified by column chromatography ( colourless oil $(0.80 \mathrm{~g}, 31 \%$ yield $):[\alpha]_{\mathrm{D}}{ }^{26}-29.01$ (c $\left.0.63, \mathrm{CH}_{3} \mathrm{OH}\right) ; \mathrm{IR} v_{\max }\left(\right.$ film): $\mathrm{cm}^{-1} 3401$, 3020, 2934, 1688, 1529, 1390; ${ }^{1} \mathrm{H}$ NMR (400 MHz, $\left.\mathrm{CDCl}_{3}\right): \delta 5.72$ - 5.64 (m, 2H), 5.18 - 5.16 (m, 1H), 4.04 (brs, 1H), $3.90(\mathrm{dd}, J=3.3,8.0 \mathrm{~Hz}, 1 \mathrm{H}), 3.65$ (d, $J=5.0 \mathrm{~Hz}, 2 \mathrm{H}), 1.96-1.91$ (m,1 $\mathrm{H}), 1.58-1.50$ (brs, 6H), 1.43 (s, 9H), 1.00 (d, $J=7.0 \mathrm{~Hz}, 3 \mathrm{H}) ;{ }^{13} \mathrm{C}$ NMR $\left(100 \mathrm{MHz}, \mathrm{CDCl}_{3}\right): \delta$ $152.1,138.3,117.0,94.3,80.7,80.2$, 66.3, 62.3, 45.1, 35.8, 29.7, 28.4, 26.2, 25.6, 10.8; HRMS calculated for $\mathrm{C}_{15} \mathrm{H}_{27} \mathrm{O}_{4} \mathrm{NNa}[\mathrm{M}+\mathrm{Na}]^{+}:$308.1832, found 308.1831.

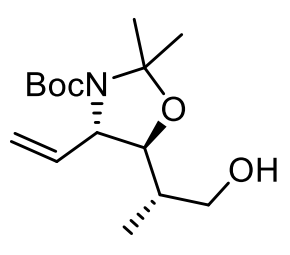

$27 \mathbf{a}$

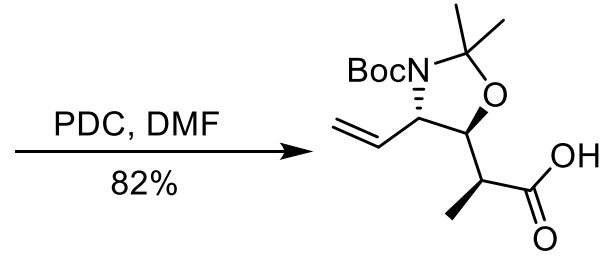

28

(S)-2-((4S,5S)-3-(tert-Butoxycarbonyl)-2,2-dimethyl-4-vinyloxazolidin-5-yl)propanoic acid (28): To a stirred solution of compound 27a $(0.65 \mathrm{~g}, 2.2 \mathrm{mmol})$ in DMF (6 mL), Pyridinium dichromate (PDC) (2.54 g, $6.84 \mathrm{mmol}$ ) was added and stirred at room temperature for $4 \mathrm{~h}$. To the reaction mixture water $(5 \mathrm{~mL})$ was added and extracted with ethyl acetate $(20 \mathrm{~mL} \times 2)$, combined the organic layers and washed with brine $(5 \mathrm{~mL})$ concentrated under reduced pressure. Purification by column chromatography (silica gel 100-200 mesh $30 \%$ ethyl acetate $-\mathrm{CH}_{2} \mathrm{Cl}_{2}$ ) 
yielded compound $28(0.56 \mathrm{~g}, 82 \%)$ as a colorless liquid. $[\alpha]_{\mathrm{D}}{ }^{26}-15.77\left(c 0.38, \mathrm{CH}_{3} \mathrm{OH}\right)$; IR $v_{\max }\left(\right.$ film): $\mathrm{cm}^{-1} 3342,3020,2935,1688,1523,1458 ;{ }^{1} \mathrm{H}$ NMR $\left(400 \mathrm{MHz}, \mathrm{CDCl}_{3}\right): \delta 5.75-5.72$ (m, 1H), 5.18(d, J=8.3 Hz, 2H), $4.10-4.07(\mathrm{~m}, 2 \mathrm{H}), 2.72-2.67(\mathrm{~m}, 1 \mathrm{H}), 1.59$ (s, 3H), 1.53 (s, $3 \mathrm{H}), 1.43(\mathrm{~s}, 9 \mathrm{H}), 1.29$ (d, $J=6.8 \mathrm{~Hz}, 3 \mathrm{H}) ;{ }^{13} \mathrm{C} \mathrm{NMR}\left(100 \mathrm{MHz}, \mathrm{CDCl}_{3}\right): \delta$ 179.1, 152.0, 137.4, 117.1, 94.6, 79.6, 63.4, 41.7, 28.4, 26.5, 26.3, 11.9; HRMS calculated for $\mathrm{C}_{15} \mathrm{H}_{25} \mathrm{O}_{5} \mathrm{NNa}$ $[\mathrm{M}+\mathrm{Na}]^{+}: 322.1625$, found 322.1625 .
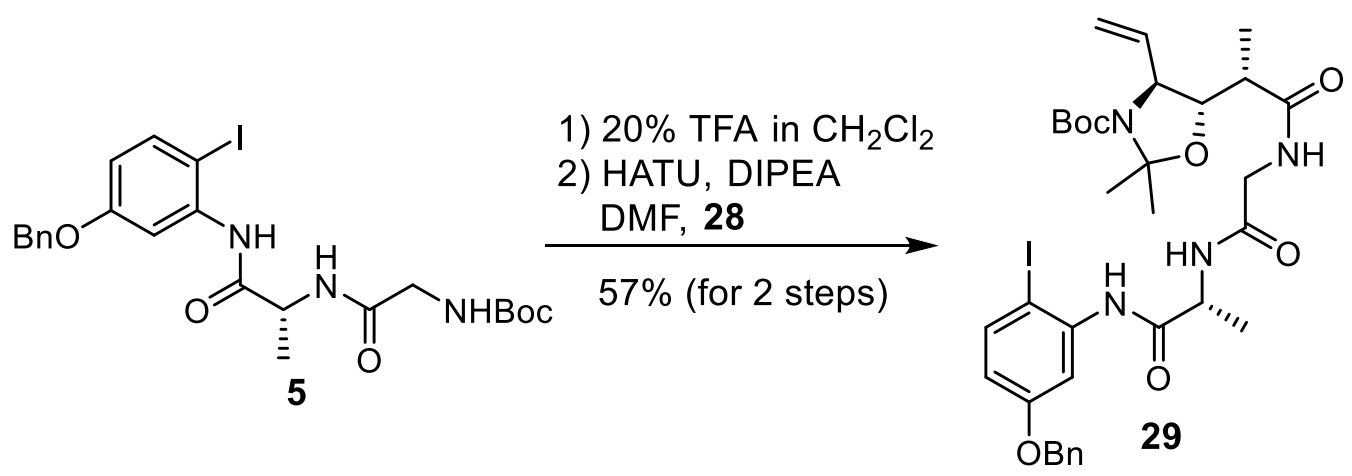

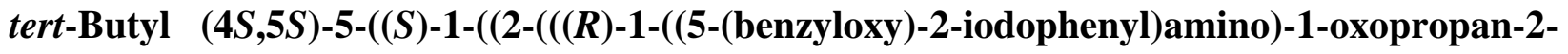
yl)amino)-2-oxoethyl)amino)-1-oxopropan-2-yl)-2,2-dimethyl-4-vinyloxazolidine-3-

carboxylate (29): The compound 29 (760 mg, 57\%) was synthesized from 5 and 28 by following similar procedure for the synthesis of compound 12. Mp $=89-90{ }^{\circ} \mathrm{C} ;[\alpha]_{\mathrm{D}}{ }^{26}+22.89(c$ $0.21, \mathrm{CH}_{3} \mathrm{OH}$ ); IR $v_{\max }\left(\right.$ film): $\mathrm{cm}^{-1} 3021,1666,1586,1518,1420,1215 ;{ }^{1} \mathrm{H}$ NMR $(400 \mathrm{MHz}$, $\left.\mathrm{CD}_{3} \mathrm{OD}\right): \delta 7.73-7.67(\mathrm{~m}, 1 \mathrm{H}), 7.59$ (brs, $\left.1 \mathrm{H}\right), 7.42-7.20(\mathrm{~m}, 7 \mathrm{H}), 6.72$ - $6.65(\mathrm{~m}, 1 \mathrm{H}), 5.89$ (ddd, $J=5.4,11.0,16.9 \mathrm{~Hz}, 1 \mathrm{H}), 5.21-5.13(\mathrm{~m}, 2 \mathrm{H}), 5.05(\mathrm{~s}, 2 \mathrm{H}), 4.65-4.52(\mathrm{~m}, 1 \mathrm{H}), 4.14$ (brs, 1H), $4.04-4.00(\mathrm{~m}, 2 \mathrm{H}), 3.85(\mathrm{~s}, 1 \mathrm{H}), 3.81-3.76(\mathrm{~m}, 1 \mathrm{H}), 3.05-2.92(\mathrm{~m}, 2 \mathrm{H}), 2.55-2.50$ (m, 1H), $1.54-1.43(\mathrm{~m}, 9 \mathrm{H}), 1.38(\mathrm{~s}, 9 \mathrm{H}), 1.24(\mathrm{~d}, J=6.8 \mathrm{~Hz}, 3 \mathrm{H}) ;{ }^{13} \mathrm{C}$ NMR (100 MHz, $\left.\mathrm{CD}_{3} \mathrm{OD}\right): \delta 179.0,173.5,173.2,172.3,170.3,164.3,161.1,158.3,140.7,140.5,138.8,138.3$, $129.7,129.2,128.8,116.1,115.5,114.4,112.5,83.8,82.2,80.8,75.9,71.4,71.3,56.7,51.3$, 47.4, 45.1, 44.5, 40.3, 29.0, 17.5, 15.4; HRMS calculated for $\mathrm{C}_{33} \mathrm{H}_{43} \mathrm{O}_{7} \mathrm{~N}_{4} \mathrm{NaI}[\mathrm{M}+\mathrm{Na}]^{+}$: 757.2069, found 757.2044. 

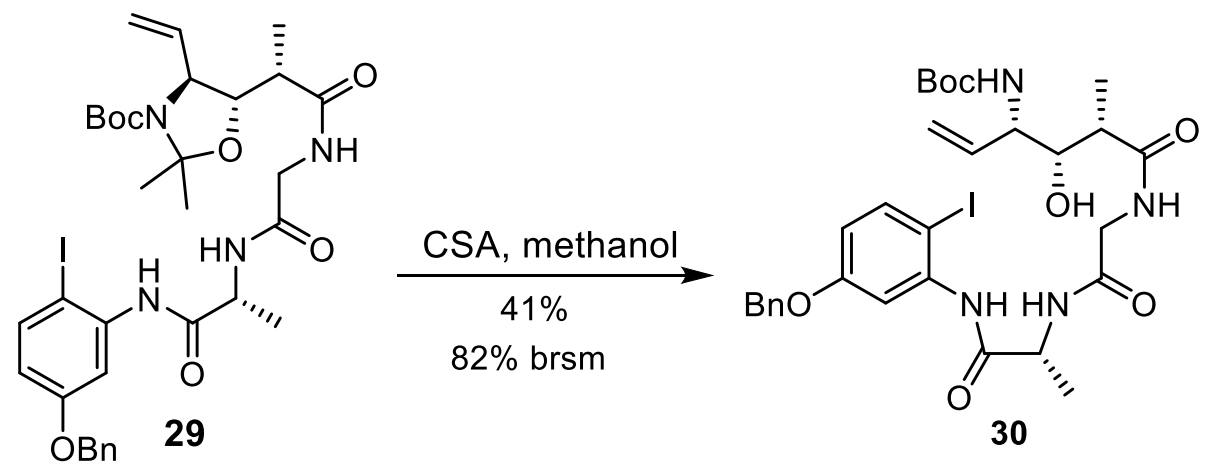

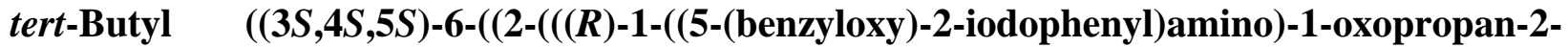
yl)amino)-2-oxoethyl)amino)-4-hydroxy-5-methyl-6-oxohex-1-en-3-yl)carbamate (30):

The compound 30 (234 mg, 41\% yield, 82\% brsm) was synthesized from 29 by following similar procedure for the synthesis of compound 4. $\mathrm{Mp}=92-93{ }^{\circ} \mathrm{C} ;[\alpha]_{\mathrm{D}}{ }^{26}+12.13\left(c 0.70, \mathrm{CH}_{3} \mathrm{OH}\right)$; IR $v_{\max }\left(\right.$ film): $\mathrm{cm}^{-1} 3322,3020,1679,1585,1520 ;{ }^{1} \mathrm{H}$ NMR $\left(400 \mathrm{MHz}, \mathrm{CD}_{3} \mathrm{OD}\right) \delta 7.69(\mathrm{~d}, J=8.6$ Hz, 1H), 7.59 (s, 1H), 7.38 - 7.30 (m, 2H), 7.36 (t, J= 7.4 Hz, 2H), 7.33 - 7.28 (m, 1H), 6.67 (dd, $J=2.7,8.8 \mathrm{~Hz}, 1 \mathrm{H}), 5.88(\mathrm{ddd}, J=5.3,10.7,16.8 \mathrm{~Hz}, 1 \mathrm{H}), 5.21-5.13(\mathrm{~m}, 2 \mathrm{H}), 5.06(\mathrm{~s}, 2 \mathrm{H})$, $4.63(\mathrm{~m}, 1 \mathrm{H}), 4.14$ (brs, 1H), $4.03-4.00$ (d, $J=17.2 \mathrm{~Hz}, 1 \mathrm{H}), 3.84-3.35$ (m, 2H), 2.53 - 2.50 $(\mathrm{m}, 1 \mathrm{H}), 1.49$ (d, $J=7.2 \mathrm{~Hz}, 3 \mathrm{H}), 1.38(\mathrm{~s}, 9 \mathrm{H}), 1.22$ (d, $J=6.9 \mathrm{~Hz}, 3 \mathrm{H}) ;{ }^{13} \mathrm{C} \mathrm{NMR}(100 \mathrm{MHz}$, $\left.\mathrm{CD}_{3} \mathrm{OD}\right): \delta 177.4,171.7,170.8,159.6,156.7,139.0,138.9,137.3,136.8,128.2,127.6,127.2$, $114.5,114.1,111.0,80.7,79.2,74.4,69.8,55.2,49.8,43.6,42.9,27.5,16.0,13.8$; HRMS calculated for $\mathrm{C}_{30} \mathrm{H}_{39} \mathrm{O}_{7} \mathrm{~N}_{4} \mathrm{NaI}[\mathrm{M}+\mathrm{Na}]^{+}:$717.1756, found 717.1755.

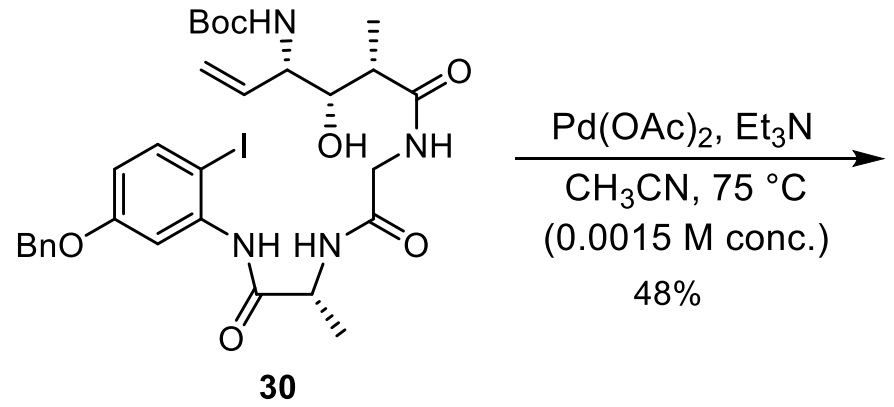

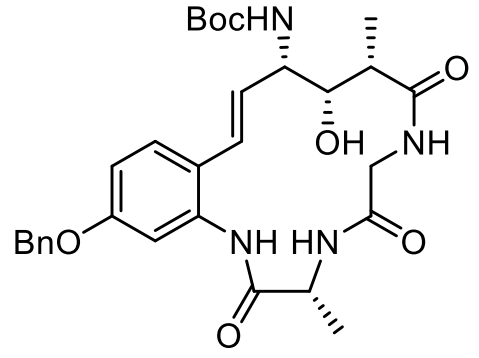

31

tert-Butyl ((3R,9S,10S,11S,E)-16-(benzyloxy)-10-hydroxy-3,9-dimethyl-2,5,8-trioxo2,3,4,5,6,7,8,9,10,11-decahydro-1H-benzo[h] $[1,4,7]$ triazacyclopentadecin-11-yl)carbamate (31): 
The compound 31 (135 mg, 48\%) was synthesized from compound $\mathbf{3 0}$ by following similar procedure for the synthesis of compound 3. $\mathrm{Mp}=136-139{ }^{\circ} \mathrm{C}$; $[\alpha]_{\mathrm{D}}{ }^{26}+9.28\left(c 0.56, \mathrm{CH}_{3} \mathrm{OH}\right)$; IR $v_{\max }\left(\right.$ film): $\mathrm{cm}^{-1} 3384,3021,2401,1657,1523,1423 ;{ }^{1} \mathrm{H}$ NMR $\left(500 \mathrm{MHz}, \mathrm{CD}_{3} \mathrm{OD}\right) \delta 7.46-$ 7.42 (m, 3H), 7.36 (t, $J=6.7 \mathrm{~Hz}, 3 \mathrm{H}), 7.30$ (d, $J=6.5 \mathrm{~Hz}, 1 \mathrm{H}), 6.99$ (brs, 1H), 6.88 (d, $J=8.0$ Hz, 1H), 6.61 (s, 1H), 5.96 (brs, 1H), 5.07 (s, 2H), 4.38 (d, $J=6.5$ Hz, 2H), 4.08 (brs, 1H), 3.70 (brs, 1H), $3.65-3.60$ (m, 1H), 2.60 (t, $J=7.1 \mathrm{~Hz}, 1 \mathrm{H}), 1.48$ (d, $J=6.9 \mathrm{~Hz}, 3 \mathrm{H}), 1.45$ (brs, 9H), $1.09(\mathrm{~d}, J=6.5 \mathrm{~Hz}, 3 \mathrm{H}) ;{ }^{13} \mathrm{C} \mathrm{NMR}\left(125 \mathrm{MHz}, \mathrm{CD}_{3} \mathrm{OD}\right): \delta 177.9,174.1,172.8,160.1,158.3$, 138.6, 136.7, 129.7, 129.4, 129.1, 128.7, 128.4, 127.6, 126.6, 115.1, 113.8, 80.4, 77.5, 71.3, 59.5, 51.9, 46.8, 44.5, 28.9, 17.0, 15.0; HRMS calculated for $\mathrm{C}_{30} \mathrm{H}_{38} \mathrm{O}_{7} \mathrm{~N}_{4} \mathrm{Na}[\mathrm{M}+\mathrm{Na}]^{+}$: 589.2633, found 589.2635 .

X-ray Crystal Structure Details of compound 31: Single crystals of compound 31 were obtained from (ethyl acetate- hexane) by slow evaporation methd. X-ray intensity data were collected on a Bruker SMART APEX II CCD diffractometer with graphite-monochromatized (Mo $\mathrm{K} \alpha=0.71073 \AA$ ) radiation at temperatures 150 (2) $\mathrm{K}$ respectively. The $\mathrm{X}$-ray generator was operated at $50 \mathrm{kV}$ and $30 \mathrm{~mA}$. A preliminary set of cell constants and an orientation matrix were calculated from three sets of 12 frames (total 36 frames). Diffraction data were collected with a $\omega$ scan width of $0.5^{\circ}$ and at different settings of $\varphi$ and $2 \theta$ with a frame time of $25 \mathrm{sec}$ keeping the sample-to-detector distance fixed at $5.00 \mathrm{~cm}$. The X-ray data acquisition was monitored by APEX II program suite. All the data were corrected for Lorentz-polarization and absorption effects using SAINT and SADABS programs integrated in APEX II program package. The structures were solved by direct method and refined by full matrix least squares, based on $F^{2}$, using SHELX-97. Molecular diagrams were generated using XSHELL program integrated in SHELXTL package.

Crystallographic data of $31\left(\mathrm{C}_{30} \mathrm{H}_{38} \mathrm{~N}_{4} \mathrm{O}_{7}\right): M=566.64$, Crystal dimensions 0.44 x 0.29 x 0.19 $\mathrm{mm}^{3}$, monoclinic, space group $C 2, a=28.205(7), b=5.7022(12), c=17.974(4) \AA, \beta=$ 91.077(4) $)^{\circ}, V=2932.5(12) \AA^{3}, Z=4, \rho_{\text {calcd }}=1.383 \mathrm{gcm}^{-3}, \mu\left(\mathrm{Mo-K} \mathrm{K}_{\alpha}\right)=0.092 \mathrm{~mm}^{-1}, F(000)=$ $1208,2 \theta_{\max }=57.58^{\circ}, T=150(2) \mathrm{K}, 18488$ reflections collected, 7297 unique reflections $\left(R_{\mathrm{int}}=0.1314\right), 4170$ observed $(I>2 \sigma(I))$ reflections, 376 refined parameters, $R$ value 0.0817 , $w R 2=0.1554$, (all data $R=0.1443, w R 2=0.1807$ ), $S=0.958$, minimum and maximum transmission 0.961 and 0.983 ; maximum and minimum residual electron densities +0.44 and - 


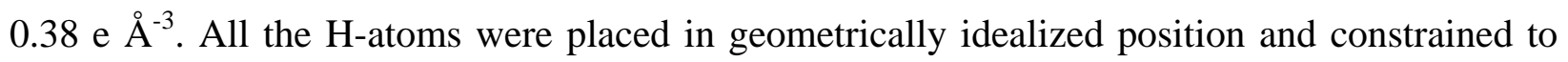
ride on their parent atoms. Crystallographic data for compound $\mathbf{3 1}$ deposited with the Cambridge Crystallographic Data Centre as supplementary publication no.CCDC 1478861

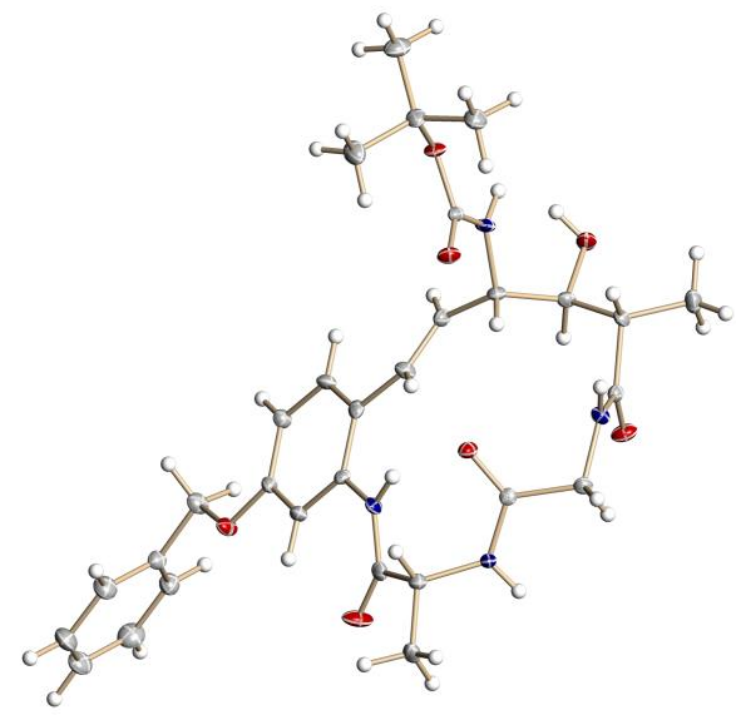

Figure 2: ORTEP diagram of compound 31

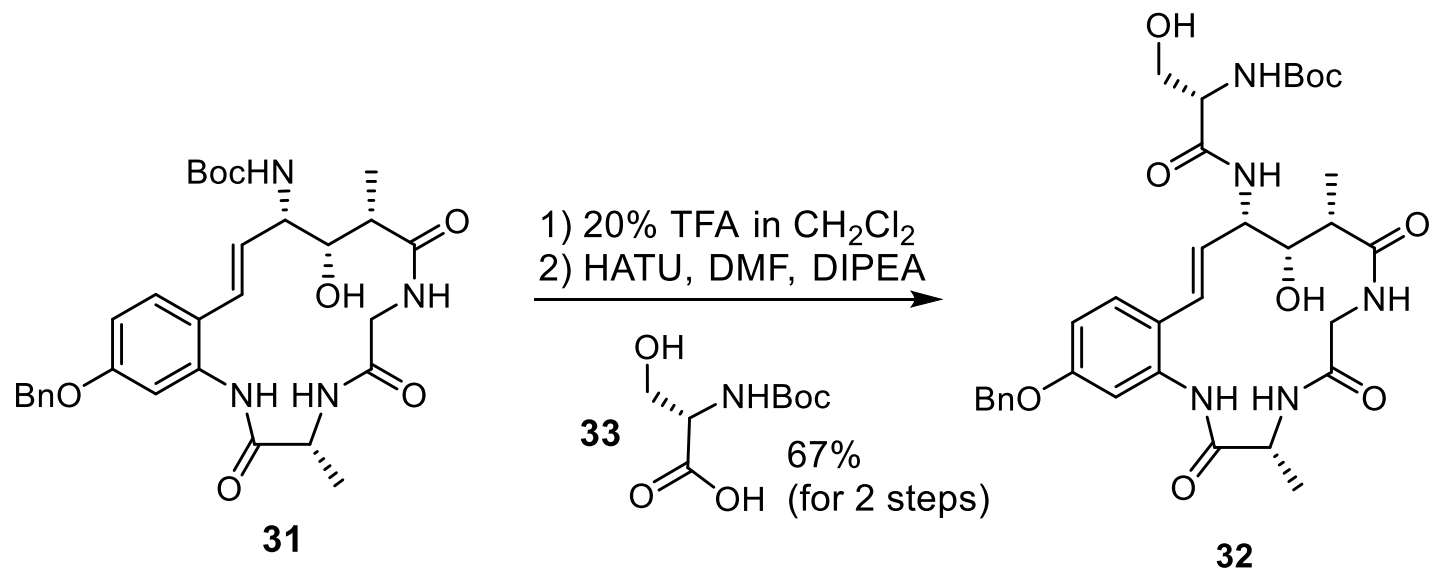

tert-Butyl ((S)-1-(((3R,9S,10S,11S,E)-16-(benzyloxy)-10-hydroxy-3,9-dimethyl-2,5,8-trioxo2,3,4,5,6,7,8,9,10,11-decahydro-1H-benzo[h][1,4,7]triazacyclopentadecin-11-yl)amino)-3-

hydroxy-1-oxopropan-2-yl)carbamate (32): To a solution of compound 31 (95 mg, 0.17 mmol) in $\mathrm{CH}_{2} \mathrm{Cl}_{2}(5 \mathrm{~mL})$ trifluoro acetic acid $(1.0 \mathrm{~mL})$ was added at $0{ }^{\circ} \mathrm{C}$ and the resulting suspension was stirred for $2 \mathrm{~h}$ at the same temperature. Reaction was monitored by TLC, and 
then concentrated. This residue was dissolved in dry DMF (3 mL), then HATU (74 mg, 0.19 mmol), DIPEA (96 $\mu \mathrm{L}, 0.44 \mathrm{mmol})$ and $N$-(tert-butoxycarbonyl)-L-serine 33 (40 $\mathrm{mg}, 0.19$ mmol) was added. The resulting solution was stirred at ambient temperature for $16 \mathrm{~h}$. Reaction mass was diluted with ethyl acetate $(15 \mathrm{~mL})$, washed with saturated solution of $\mathrm{NaHCO}_{3}(5 \mathrm{~mL})$, $\mathrm{H}_{2} \mathrm{O}(5 \mathrm{~mL})$. The organic layer was dried over $\mathrm{Na}_{2} \mathrm{SO}_{4}$ and the crude material obtained after removal of the solvent was purified by column chromatography (silica gel 230-400 mesh 4\% methanol $\left.-\mathrm{CH}_{2} \mathrm{Cl}_{2}\right)$ to afford $32(73 \mathrm{mg}, 67 \%)$ as off white solid. $\mathrm{Mp}=98-99{ }^{\circ} \mathrm{C}$; $[\alpha]_{\mathrm{D}}{ }^{26}+$ 16.77 (c 0.28, $\left.\mathrm{CH}_{3} \mathrm{OH}\right) ;{ }^{1} \mathrm{H} \mathrm{NMR}\left(400 \mathrm{MHz}, \mathrm{CD}_{3} \mathrm{OD}\right) \delta 7.46-7.30(\mathrm{~m}, 6 \mathrm{H}), 6.95$ - $6.88(\mathrm{~m}, 2 \mathrm{H})$, $6.64(\mathrm{~d}, J=8.7 \mathrm{~Hz}, 1 \mathrm{H}), 6.09-6.03(\mathrm{~m}, 1 \mathrm{H}), 5.07(\mathrm{~s}, 2 \mathrm{H}), 4.71(\mathrm{~d}, J=7.3 \mathrm{~Hz}, 1 \mathrm{H}), 4.36-4.32$ $(\mathrm{m}, 1 \mathrm{H}), 4.16-4.14(\mathrm{~m}, 1 \mathrm{H}), 4.02(\mathrm{~d}, J=14.7 \mathrm{~Hz}, 1 \mathrm{H}), 3.79-3.69(\mathrm{~m}, 4 \mathrm{H}), 2.50(\mathrm{dd}, J=7.1$, $9.0 \mathrm{~Hz}, 1 \mathrm{H}), 1.48(\mathrm{~d}, J=7.1 \mathrm{~Hz}, 3 \mathrm{H}), 1.45(\mathrm{~s}, 9 \mathrm{H}), 1.13(\mathrm{~d}, J=6.4 \mathrm{~Hz}, 3 \mathrm{H}) ;{ }^{13} \mathrm{C} \mathrm{NMR}(100$ $\left.\mathrm{MHz}, \mathrm{CD}_{3} \mathrm{OD}\right): \delta 176.5,172.9,171.4,171.3,158.7,137.0,135.3,128.3,128.1,127.5,127.2$, $126.1,125.3,113.7,112.2$, 79.6, 75.6, 69.7, 61.9, 56.9, 56.3, 50.5, 45.5, 42.5, 27.3, 15.4, 13.6; HRMS calculated for $\mathrm{C}_{33} \mathrm{H}_{43} \mathrm{O}_{9} \mathrm{~N}_{5} \mathrm{Na}[\mathrm{M}+\mathrm{Na}]^{+}: 676.2953$, found 676.2945.

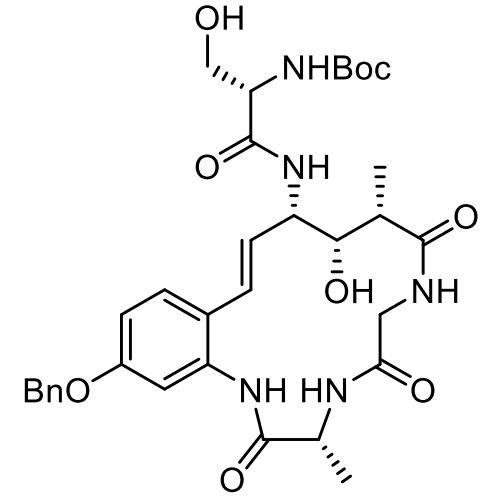

32

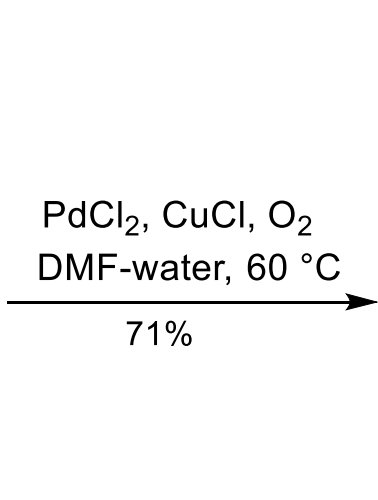

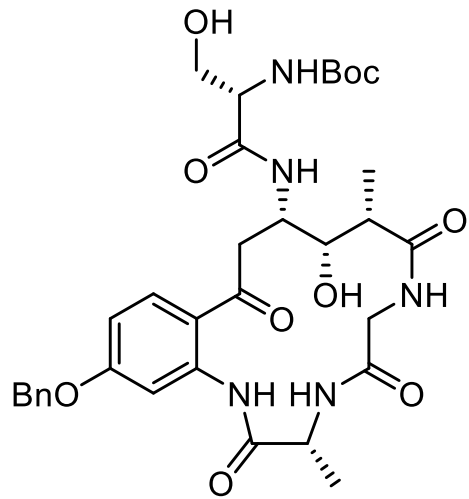

34

tert-Butyl

((S)-1-(((3R,9S,10S,11S)-16-(benzyloxy)-10-hydroxy-3,9-dimethyl-2,5,8,13tetraoxo-2,3,4,5,6,7,8,9,10,11,12,13-dodecahydro-1H-benzo[h][1,4,7]triazacyclopentadecin-

11-yl)amino)-3-hydroxy-1-oxopropan-2-yl)carbamate (34): The compound 34 (13 mg, 71\%) was synthesized from compound $\mathbf{3 2}$ as a white color solid by following similar procedure for the synthesis of 15. $\mathrm{Mp}=258-260{ }^{\circ} \mathrm{C} ;[\alpha]_{\mathrm{D}}{ }^{26}+10.76\left(c 0.13, \mathrm{CH}_{3} \mathrm{OH}\right) ;{ }^{1} \mathrm{H}$ NMR $(500 \mathrm{MHz}$, DMSO-d $_{6}$ ): $\delta 11.99$ (brs, 1H), $8.80(\mathrm{~d}, J=6.1 \mathrm{~Hz}, 1 \mathrm{H}), 8.15(\mathrm{~s}, 1 \mathrm{H}), 7.87$ (d, $\left.J=8.4 \mathrm{~Hz}, 1 \mathrm{H}\right)$, $7.47-7.27(\mathrm{~m}, 7 \mathrm{H}), 7.28(\mathrm{~d}, J=7.1 \mathrm{~Hz}, 1 \mathrm{H}), 6.99(\mathrm{~d}, J=7.6 \mathrm{~Hz}, 1 \mathrm{H}), 6.84(\mathrm{~d}, J=8.4 \mathrm{~Hz}, 1 \mathrm{H})$, 5.49 (brs, 1H), $5.21(\mathrm{~s}, 2 \mathrm{H}), 5.09(\mathrm{~s}, 1 \mathrm{H}), 4.82$ (brs, 1H), $4.34(\mathrm{~d}, J=6.9 \mathrm{~Hz}, 1 \mathrm{H}), 4.18-4.10$ (m, 
1H), $3.91-3.81(\mathrm{~m}, 2 \mathrm{H}), 3.79(\mathrm{~d}, J=15.3 \mathrm{~Hz}, 2 \mathrm{H}), 3.60-3.28(\mathrm{~m}, 2 \mathrm{H}), 2.87(\mathrm{~d}, J=17.1 \mathrm{~Hz}$, $1 \mathrm{H}), 2.34-2.20(\mathrm{~m}, 1 \mathrm{H}), 1.41(\mathrm{~s}, 9 \mathrm{H}), 1.37(\mathrm{~d}, J=6.5 \mathrm{~Hz}, 3 \mathrm{H}), 1.02(\mathrm{~m}, \mathrm{~d}, J=6.8 \mathrm{~Hz}, 3 \mathrm{H}) ;{ }^{13} \mathrm{C}$ NMR (125 MHz, DMSO-d $\left.{ }_{6}\right): \delta 201.6,173.5,171.3,169.9,169.3,162.7,155.3,141.0,136.3$, 133.9, 128.5, 128.4, 128.6, 128.1, 127.7, 127.6, 117.4, 109.1, 105.8, 78.3, 72.2, 57.5, 49.9, 46.01, 43.1, 42.9, 28.2, 16.1, 13.8. HRMS calculated for $\mathrm{C}_{33} \mathrm{H}_{43} \mathrm{O}_{10} \mathrm{~N}_{5} \mathrm{Na}[\mathrm{M}+\mathrm{Na}]^{+}: 692.2902$, found 692.2892 .
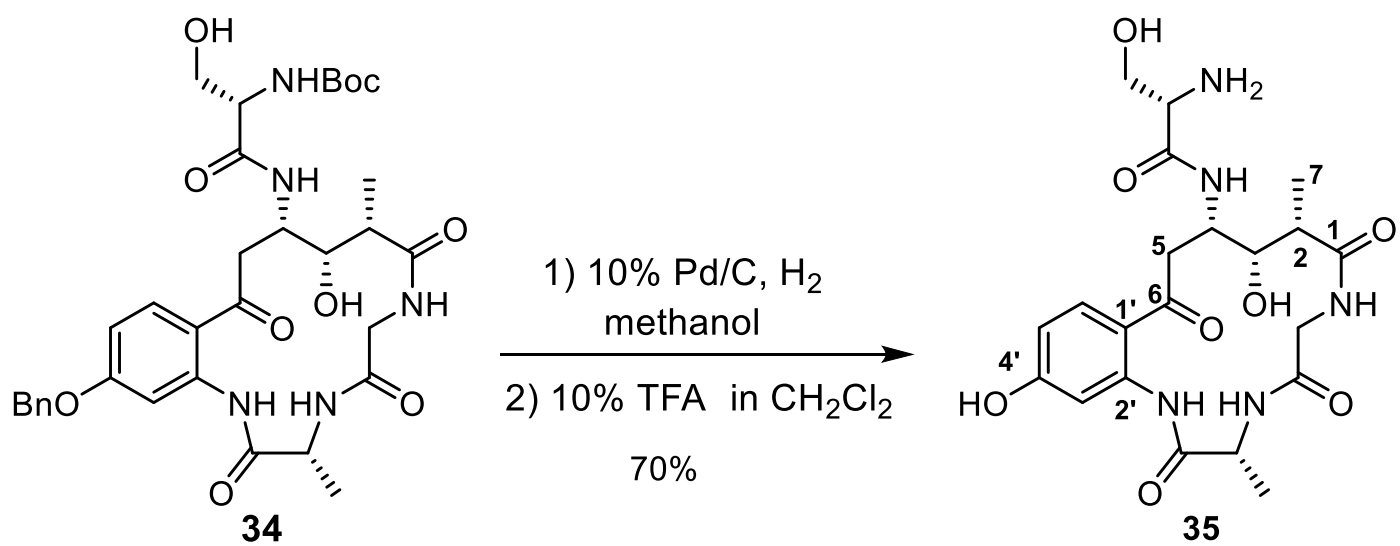

(S)-2-Amino-N-((3R,9S,10S,11S)-10,16-dihydroxy-3,9-dimethyl-2,5,8,13-tetraoxo-

\section{2,3,4,5,6,7,8,9,10,11,12,13-dodecahydro-1H-benzo[h][1,4,7]triazacyclopentadecin-11-yl)-3-}

hydroxypropanamide (35): To a solution of compound $\mathbf{3 4}(9 \mathrm{mg}, 0.013 \mathrm{mmol})$ in methanol (3 $\mathrm{mL}), 10 \% \mathrm{Pd} / \mathrm{C}(\sim 3 \mathrm{mg})$ was added and stirred under $\mathrm{H}_{2}$ atmosphere for $2 \mathrm{~h}$. The reaction mixture was then filtered through silica gel column, concentrated to afford phenolic compound. The phenolic compound was dissolved in $\mathrm{CH}_{2} \mathrm{Cl}_{2}(3 \mathrm{~mL})$, TFA $(0.3 \mathrm{~mL})$ was added at $0{ }^{\circ} \mathrm{C}$ and the resulting suspension was stirred for $2 \mathrm{~h}$ at the same temperature. Concentrated the reaction mixture and azeotroped with diethyl ether $(3 \mathrm{~mL} \times 3)$ and dried under vacuum to afford compound 35 (4.5 mg, 70\% for 2 steps) as off white solid. $[\alpha]_{\mathrm{D}}{ }^{26}+9.99\left(c 0.23, \mathrm{CH}_{3} \mathrm{OH}\right)$; HRMS calculated for $\mathrm{C}_{21} \mathrm{H}_{30} \mathrm{O}_{8} \mathrm{~N}_{5}[\mathrm{M}+\mathrm{H}]^{+}: 480.2089$, found 480.2086 . 
Table 3: ${ }^{1} \mathrm{H}$ chemical shift data of $\mathbf{2}$ and $\mathbf{3 5}$ in comparison to the reported values ${ }^{6}$

\begin{tabular}{|c|c|c|}
\hline $\begin{array}{l}{ }^{1} \mathrm{H} \text { NMR Values of } \\
\text { Solomonamide B } \\
\text { (Natural Product) }\end{array}$ & $\begin{array}{c}{ }^{1} \mathrm{H} \text { NMR Values of Proposed } \\
\text { structure of Solomonamide B } 2 \\
\text { (Synthesized) }\end{array}$ & $\begin{array}{c}{ }^{1} \text { H NMR Values of Revised } \\
\text { structure of Solomonamide B } \\
\mathbf{3 5} \text { (Synthesized) }\end{array}$ \\
\hline $1.08(\mathrm{~d}, J=7.2,3 \mathrm{H})$ & $0.95(\mathrm{~d}, J=6.1 \mathrm{~Hz}, 3 \mathrm{H})$ & $1.08(\mathrm{~d}, J=6.8 \mathrm{~Hz}, 3 \mathrm{H})$ \\
\hline $1.36(\mathrm{~d}, J=7.2,3 \mathrm{H})$ & $1.38(\mathrm{~d}, J=7.1 \mathrm{~Hz}, 3 \mathrm{H})$ & $1.36(\mathrm{~d}, J=7.3,3 \mathrm{H})$ \\
\hline $2.35(\mathrm{~m}, 1 \mathrm{H})$ & 2.73 (brs, $1 \mathrm{H})$ & $2.35(\mathrm{~m}, 1 \mathrm{H})$ \\
\hline $\begin{array}{l}2.87 \text { (br dd, } J=17.6,1.7 \\
1 \mathrm{H})\end{array}$ & $2.90(\mathrm{brd}, 1 \mathrm{H})$ & $2.84(\mathrm{br} \mathrm{dd}, J=16.4,1.5 \mathrm{~Hz} 1 \mathrm{H})$ \\
\hline 3.34 (ovl, $1 \mathrm{H})$ & Might be in moisture peak & $3.34(\mathrm{ovl}, 1 \mathrm{H})$ \\
\hline 3.39 (ovl, $1 \mathrm{H})$ & Might be in moisture peak & 3.39 (ovl, 1H) \\
\hline 3.69 (brs, 2H) & 3.70 (brs, 2H) & 3.64 (brs, 1H) $3.77(\mathrm{dd}, 1 \mathrm{H})$ \\
\hline $3.78(\mathrm{dd}, J=15.7,3.4,1 \mathrm{H})$ & 3.58 (brs, 1H) & $3.82(\mathrm{dd}, J=15.7,3.4,1 \mathrm{H})$ \\
\hline $3.98(\mathrm{~m} \mathrm{1H})$ & $3.93(\mathrm{brs}, 1 \mathrm{H})$ & $4.01(\mathrm{~m} \mathrm{1H})$ \\
\hline $4.19(\mathrm{dd}, J=15.7,7.1,1 \mathrm{H})$ & $4.20-4.06(\mathrm{~m}, 1 \mathrm{H})$ & $4.14(\mathrm{dd}, J=15.6,6.9,1 \mathrm{H})$ \\
\hline 4.29 (quint, $J=7.1,1 \mathrm{H}$ ) & & 4.32 (quint, $J=7.3,1 \mathrm{H}$ ) \\
\hline 4.52 br dt $(9.5,1.7)$ & $4.51(\mathrm{brs}, 1 \mathrm{H})$ & $4.49 \mathrm{br} \mathrm{dt}(1 \mathrm{H})$ \\
\hline $5.53(\mathrm{br} \mathrm{d}, J=5.3 \mathrm{~Hz}, 1 \mathrm{H})$ & $4.79(\mathrm{dd}, \mathrm{J}=9.0,14.9 \mathrm{~Hz}, 1 \mathrm{H})$ & $5.54(\mathrm{~d}, J=5.3 \mathrm{~Hz}, 1 \mathrm{H})$ \\
\hline $6.57(\mathrm{dd}, J=8.8,2.1,1 \mathrm{H})$ & $5.58-5.34(\mathrm{~m}, 2 \mathrm{H})$ & $6.58(\mathrm{dd}, J=8.8,2.0,1 \mathrm{H})$ \\
\hline $7.30($ br dd, $J=7.0,3.4,1 \mathrm{H})$ & $6.56(\mathrm{~d}, J=8.1 \mathrm{~Hz}, 1 \mathrm{H})$ & $7.42(\mathrm{NH}, 1 \mathrm{H})$ \\
\hline $7.77(\mathrm{~d}, J=8.8,1 \mathrm{H})$ & $7.32-7.13(\mathrm{~m}, 1 \mathrm{H})$ & $7.75(\mathrm{~d}, J=8.8,1 \mathrm{H})$ \\
\hline $7.92(\mathrm{~d}, J=2.1,1 \mathrm{H})$ & $7.68(\mathrm{~d}, J=8.3 \mathrm{~Hz}, 1 \mathrm{H})$ & $7.94(\mathrm{~d}, J=2.0,1 \mathrm{H})$ \\
\hline $7.98(\mathrm{~d}, J=9.5,1 \mathrm{H})$ & $7.84(\mathrm{~d}, J=8.3 \mathrm{~Hz}, 1 \mathrm{H})$ & $8.06(\mathrm{~d}, J=8.31 \mathrm{~Hz}, 1 \mathrm{H})$ \\
\hline $8.08(\mathrm{br} \mathrm{d}, J=4.4,2 \mathrm{H})$ & $8.11(\mathrm{brs}, 1 \mathrm{H})$ & $8.12(2 \mathrm{H}) \mathrm{NH}_{2}$ \\
\hline $8.79(\mathrm{~d}, J=7.2,1 \mathrm{H})$ & 8.05 (brs, 2H) & $8.83(\mathrm{~d}, J=7.3,1 \mathrm{H}) \mathrm{NH}$ \\
\hline $10.7 \mathrm{~s}$ & 9.06 (brs, $1 \mathrm{H})$ & $10.74 \mathrm{~s}$ \\
\hline \multirow[t]{2}{*}{$11.5 \mathrm{~s}$} & 10.72 (brs, $1 \mathrm{H})$ & $12.0 \mathrm{~s}$ \\
\hline & 12.49 (brs, $1 \mathrm{H})$ & \\
\hline
\end{tabular}


Table 4: ${ }^{13} \mathrm{C}$ NMR chemical shift data of $\mathbf{2}$ and $\mathbf{3 5}$ in comparison to the reported values ${ }^{6}$

\begin{tabular}{|c|c|c|c|}
\hline Residue & $\begin{array}{c}{ }^{13} \mathrm{C} \text { NMR Values of } \\
\text { Solomonamide B } \\
\text { (Natural Product) }\end{array}$ & $\begin{array}{l}{ }^{13} \mathrm{C} \text { NMR Values of } \\
\text { Proposed structure of } \\
\text { Solomonamide B } 2 \\
\text { (Synthesized) }\end{array}$ & $\begin{array}{c}{ }^{1} \mathrm{H} \text { NMR Values of } \\
\text { Revised structure of } \\
\text { Solomonamide B } 35 \\
\text { (Synthesized) }\end{array}$ \\
\hline \multicolumn{4}{|l|}{ Glycine } \\
\hline Carbonyl & 169.0 & 169.8 & 169.1 \\
\hline $\mathrm{CH}_{2}$ & 42.4 & 42.0 & 42.6 \\
\hline \multicolumn{4}{|l|}{ D-Alanine } \\
\hline Carbonyl & 171.2 & 172.1 & 171.2 \\
\hline $\mathrm{CH}$ & 49.7 & 50.7 & 49.9 \\
\hline Methyl & 16.0 & 16.9 & 16.2 \\
\hline \multicolumn{4}{|l|}{ L-Serine } \\
\hline Carbonyl & 166.7 & 165.6 & 166.7 \\
\hline $\mathrm{CH}$ & 53.6 & 53.6 & 54.1 \\
\hline $\mathrm{CH}_{2}$ & 60.3 & 60.6 & 60.6 \\
\hline \multicolumn{4}{|l|}{ AHMOA } \\
\hline Carbonyl & 173.2 & 172.6 & 173.3 \\
\hline $\begin{array}{ll}2 & \mathrm{CH} \text { attached to } \\
\text { methyl } & \end{array}$ & 45.5 & 45.2 & 45.6 \\
\hline $\begin{array}{ll}3 & \mathrm{CH} \text { attached to } \\
\mathrm{OH} & \end{array}$ & 72.2 & 71.4 & 72.3 \\
\hline $\begin{array}{ll}4 & \mathrm{CH} \text { attached to } \\
\mathrm{NH} & \end{array}$ & 48.0 & 45.2 & 48.6 \\
\hline $\mathrm{CH}_{2}$ & 41.2 & 42.3 & 41.2 \\
\hline Keto carbonyl & 201.1 & 200.2 & 201.0 \\
\hline $7 \quad$ Methyl & 13.6 & 13.6 & 13.8 \\
\hline \multicolumn{4}{|l|}{ Aromatic } \\
\hline 1 & 115.8 & 113.9 & 116.0 \\
\hline 2 & 141.3 & 142.4 & 141.3 \\
\hline 3 & 106.1 & 105.6 & 106.3 \\
\hline 4 & 162.9 & 163.3 & 162.9 \\
\hline 5 & 110.0 & 110.1 & 110.2 \\
\hline 6 & 132.9 & 133.6 & 132.9 \\
\hline
\end{tabular}




\section{References:}

1. Sheppard, G. S.; Wang, J.; Kawai, M.; BaMaung, N. Y.; Craig, R. A.; Erickson, S. A.; Lynch, L.; Patel, J.; Yang, F.; Searle, X. B.; Lou, P.; Park, C.; Kim, K. H.; Henkin, J.; Lesniewski, R. Bioorg. Med. Che. Lett. 2004, 14, 865.

2. Hatakeyama, K.; Ohmori, K.; Suzuki, K. Synlett 2005, 1311.

3. Asif, K.; Himaja, M.; Ramana, M. V.; Sikarwar, M. S. Asian J. Chem. 2012, 24, 2739.

4. Yoo, D.; Oh, J. S.; Lee, D.-W.; Y Kim, G. J. Org. Chem. 2003, 68, 2979.

5. Kashinath, K.; Dhara, S.; Reddy, D. S. Org. Lett. 2015, 17, 2090.

6. Festa, C.; De Marino, S.; Sepe, V.; Auria, M. V. D.; Bifulco, G.; Débitus, C.; Bucci, M.; Vellecco, V.; Zampella, A. Org. Lett. 2011, 13, 1532.

7. a) Karplus, M. J. Chem. Phys. 1959, 30, 11. b) Karplus, M. J. Am. Chem. Soc. 1963, 85, 2870. c) Minch, M. J. Concepts Magn. Reson.1994, 6, 41.

8. a) Evans, D. A.; Bartroli, J.; Shih, T. L. J. Am. Chem. Soc. 1981, 103, 2127. b) Long, B.; Tang, S.; Chen, L.; Qu, S.; Chen, B.; Liu, J.; Maguire, A. R.; Wang, Z.; Liu, Y.; Zhang, H.; Xu, Z.; Ye, T. Chem. Commun. 2013, 49, 2977.

9. Ding, F.; William, R.; Leow, M. L.; Chai, H.; Fong, J. Z. M.; Liu, X.-W. Org. Lett. 2014, $16,26$. 


\section{${ }^{1} \mathrm{H}$ NMR (400 MHz, $\mathrm{CDCl}_{3}$ ) of compound 8}

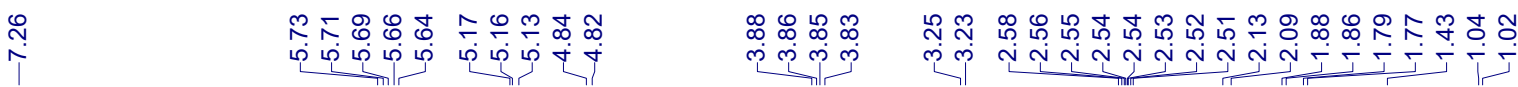

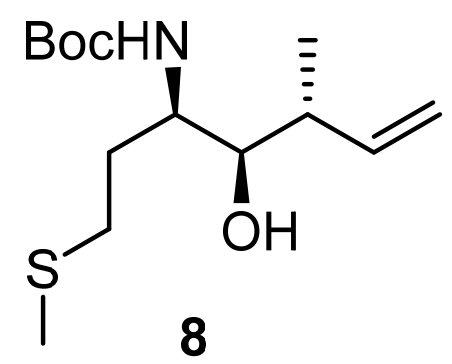




\section{${ }^{13} \mathrm{C}$ NMR (100 MHz, $\left.\mathrm{CDCl}_{3}\right)$ of compound 8}

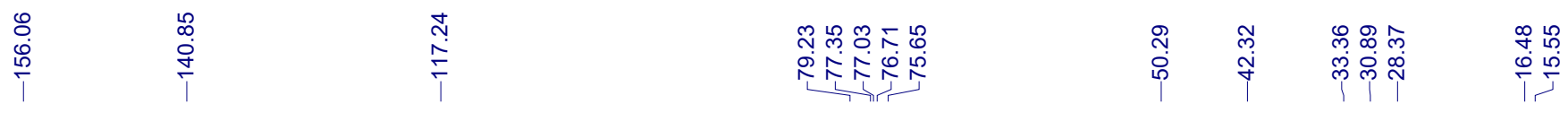

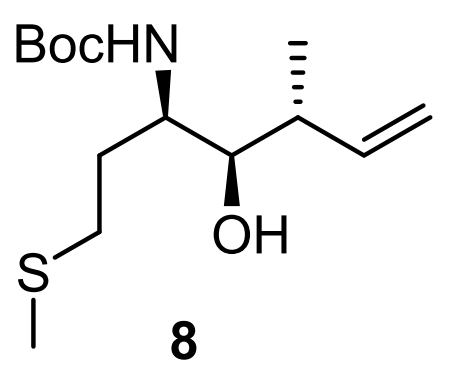

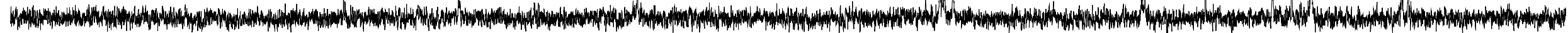

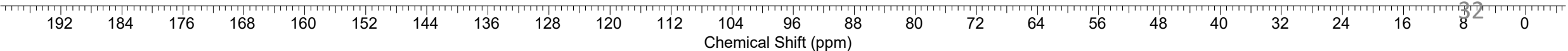




\section{${ }^{1} \mathrm{H}$ NMR (400 MHz, $\mathrm{CDCl}_{3}$ ) of compound 9}

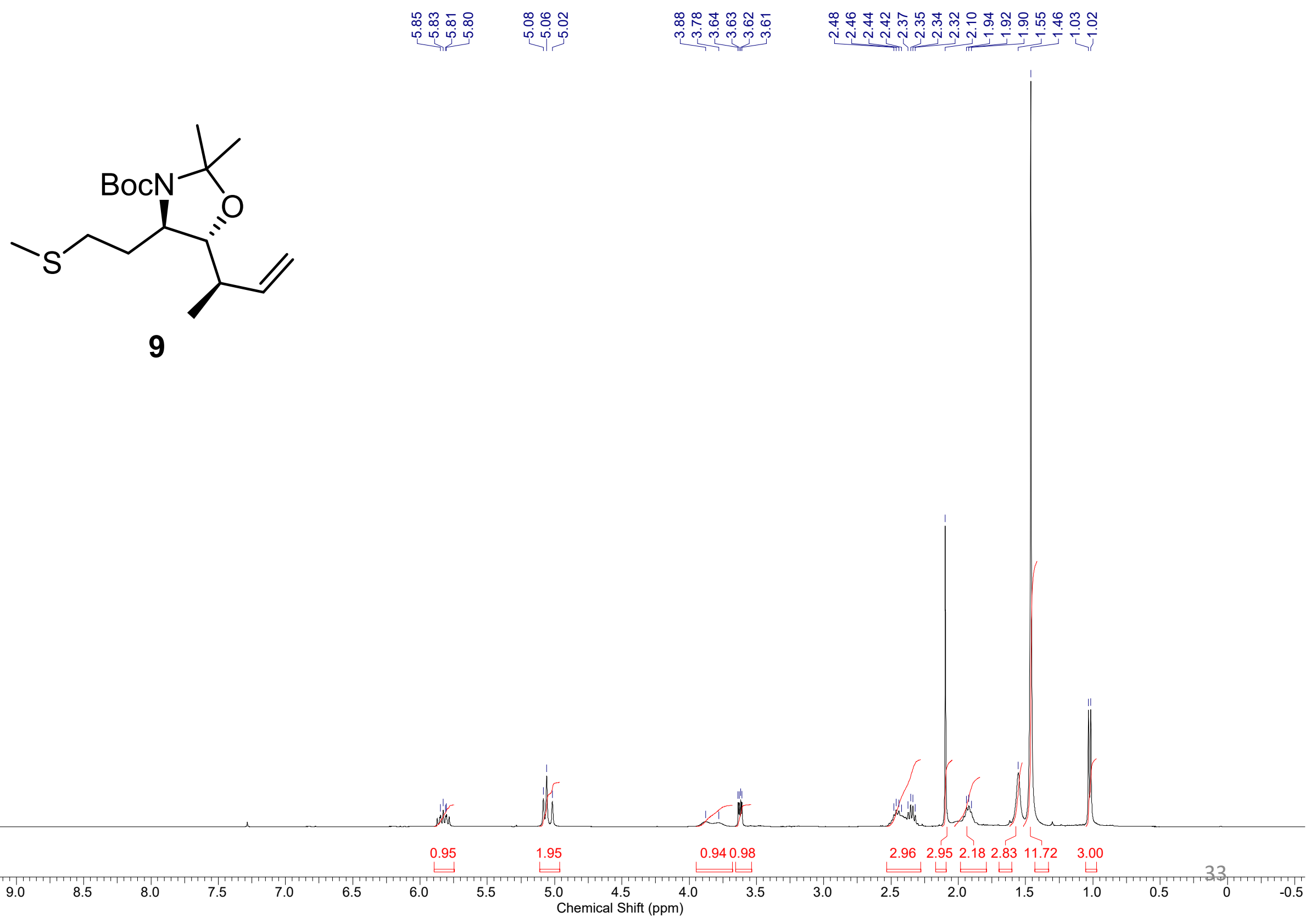


${ }^{13} \mathrm{C}$ NMR (100 $\left.\mathrm{MHz}, \mathrm{CDCl}_{3}\right)$ of compound 9

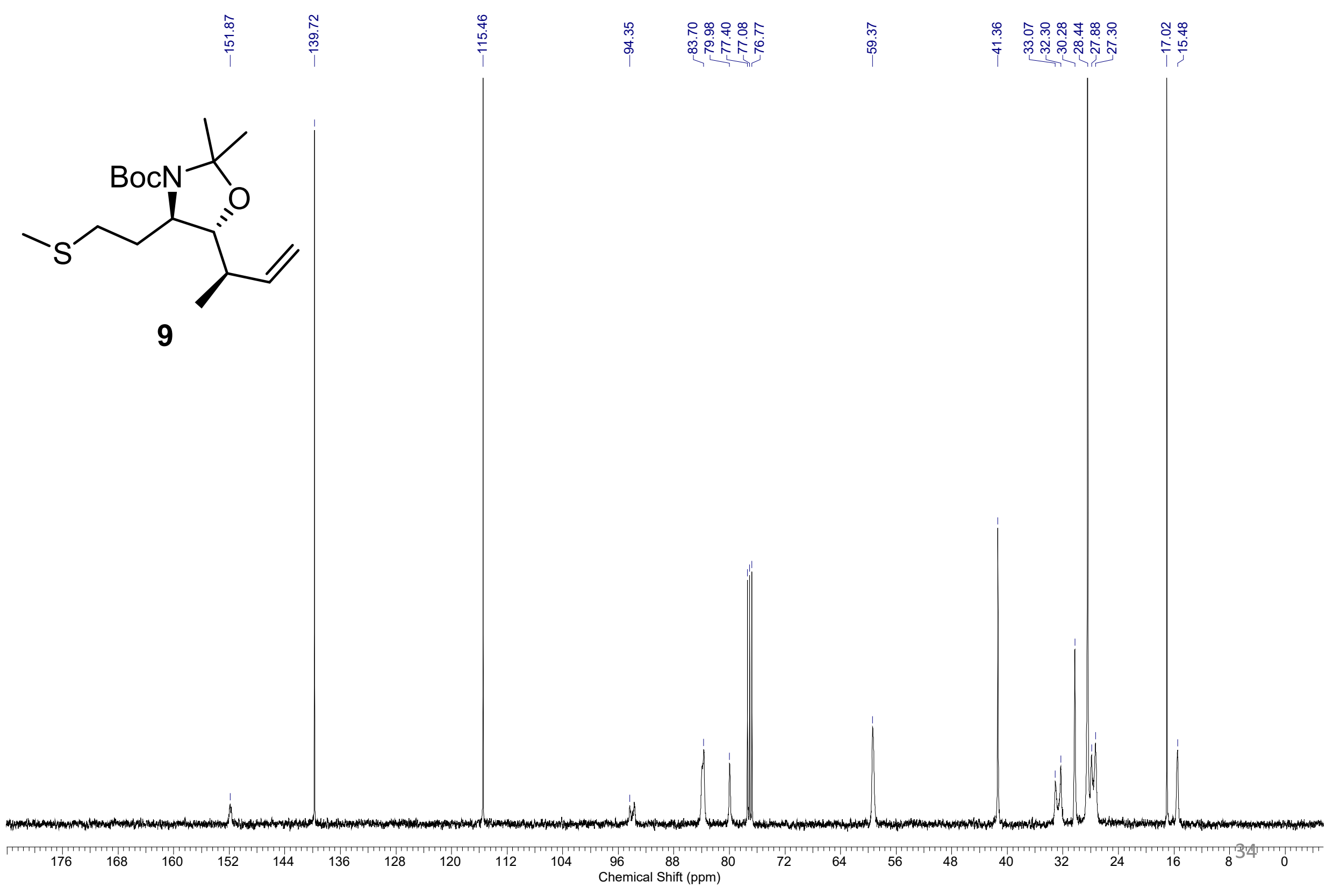




\section{${ }^{1} \mathrm{H}$ NMR (400 MHz, $\mathrm{CDCl}_{3}$ ) of compound 10}

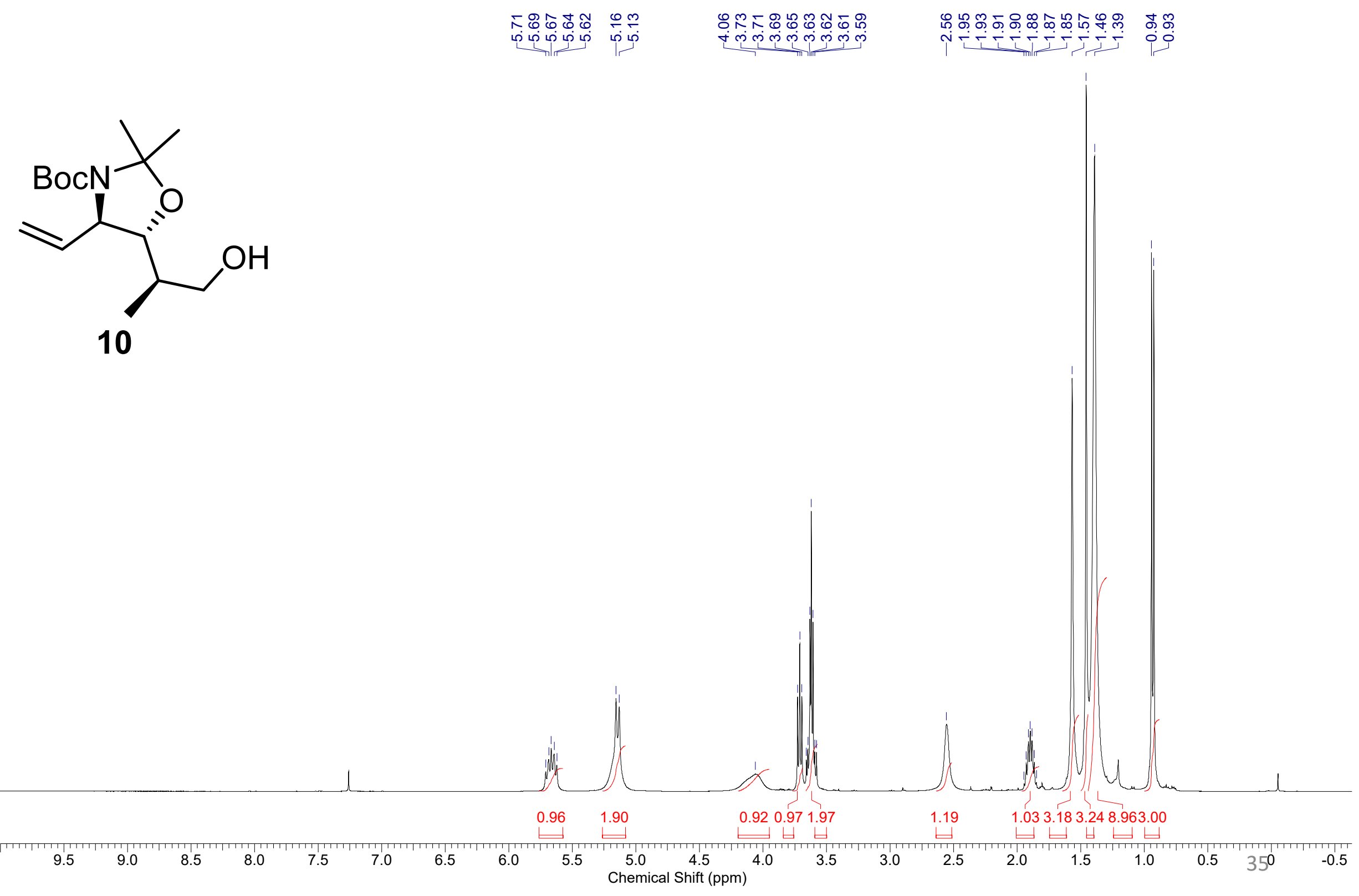


${ }^{13} \mathrm{C}$ NMR (100 MHz, $\left.\mathrm{CDCl}_{3}\right)$ of compound 10

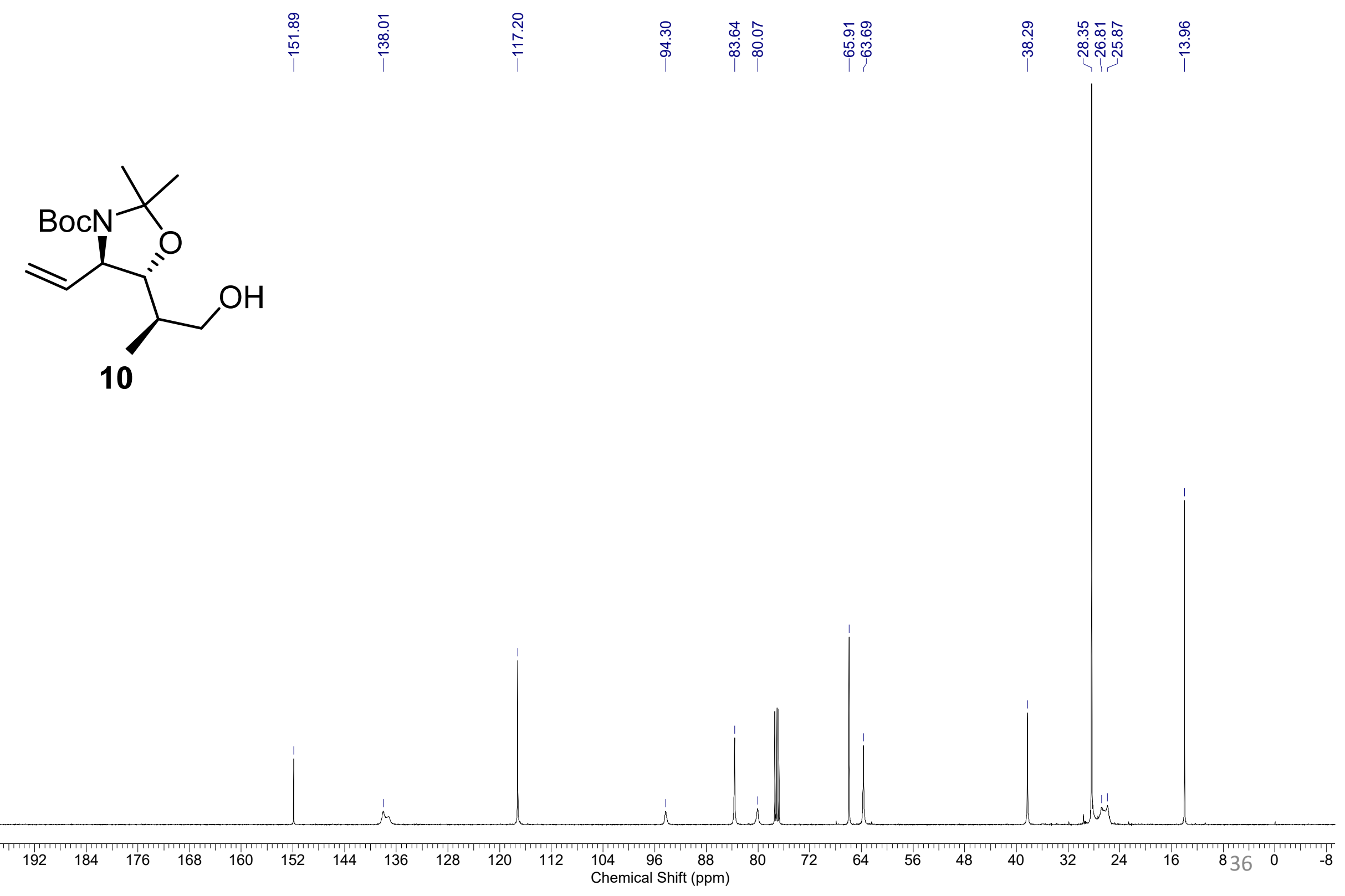


${ }^{1} \mathrm{H}$ NMR (400 MHz, $\mathrm{CDCl}_{3}$ ) of compound 6

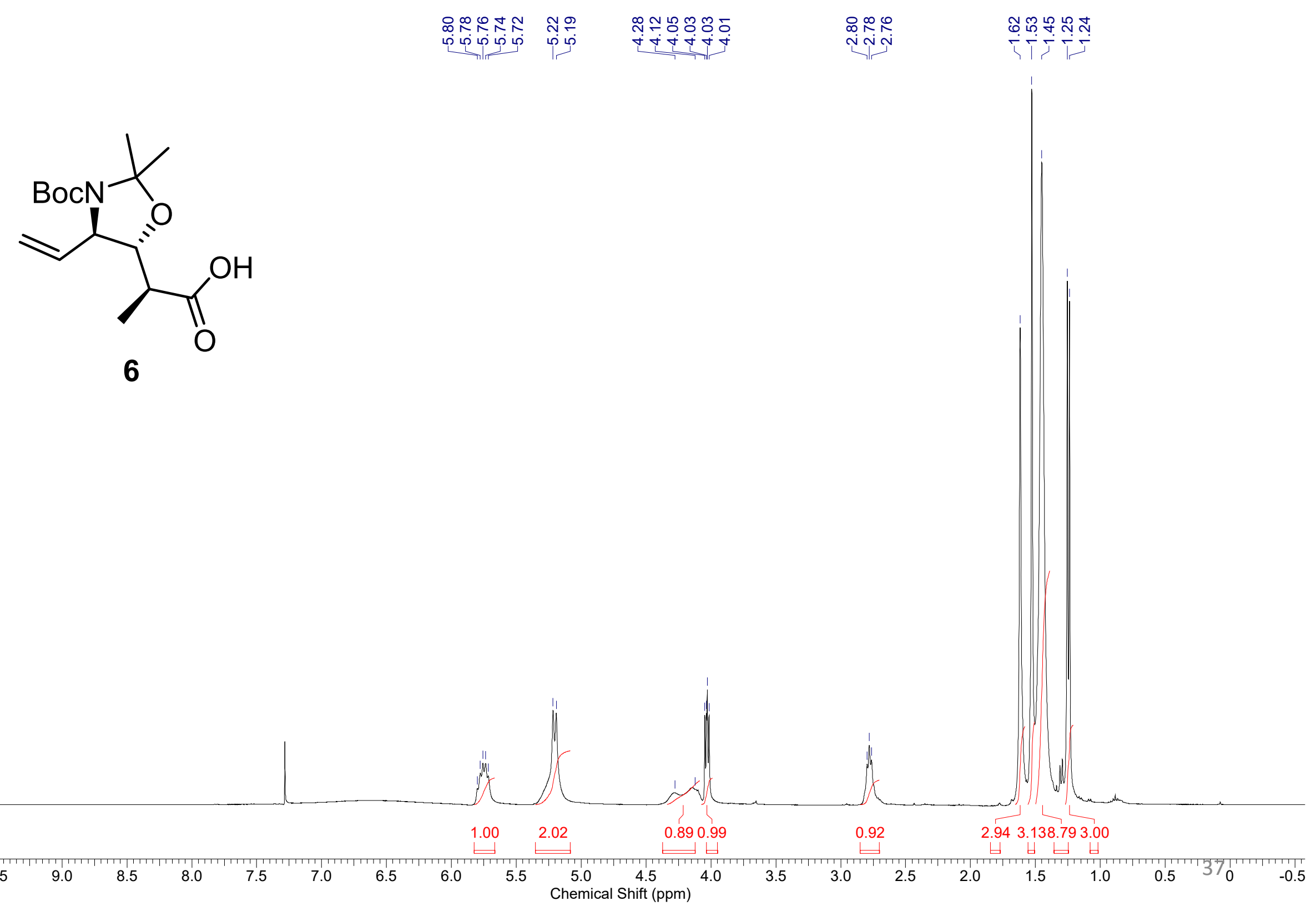


${ }^{13} \mathrm{C}$ NMR (100 MHz, $\left.\mathrm{CDCl}_{3}\right)$ of compound 6

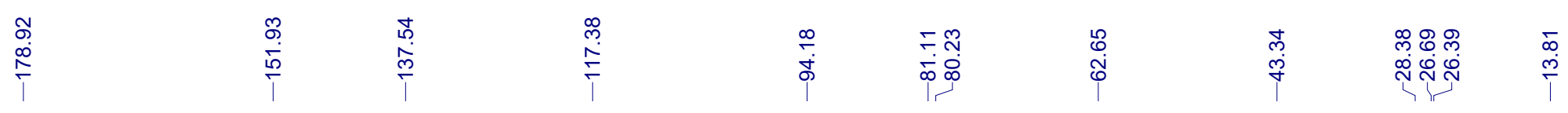

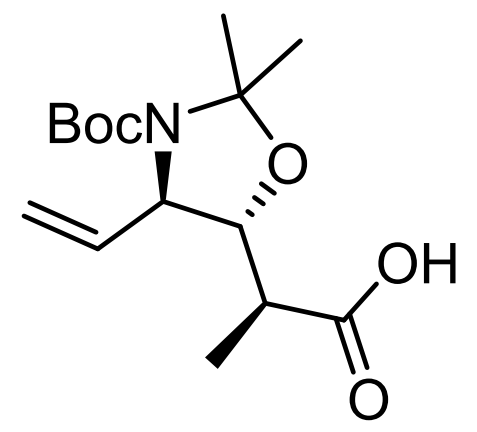

6

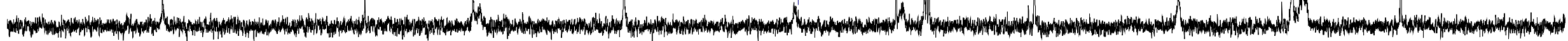

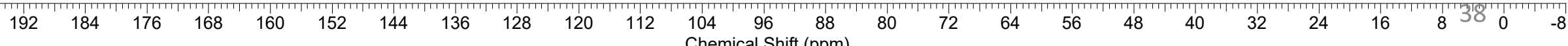
Chemical Shift (ppm) 
${ }^{1} \mathrm{H}$ NMR (400 MHz, DMSO-d 6 ) of compound 5

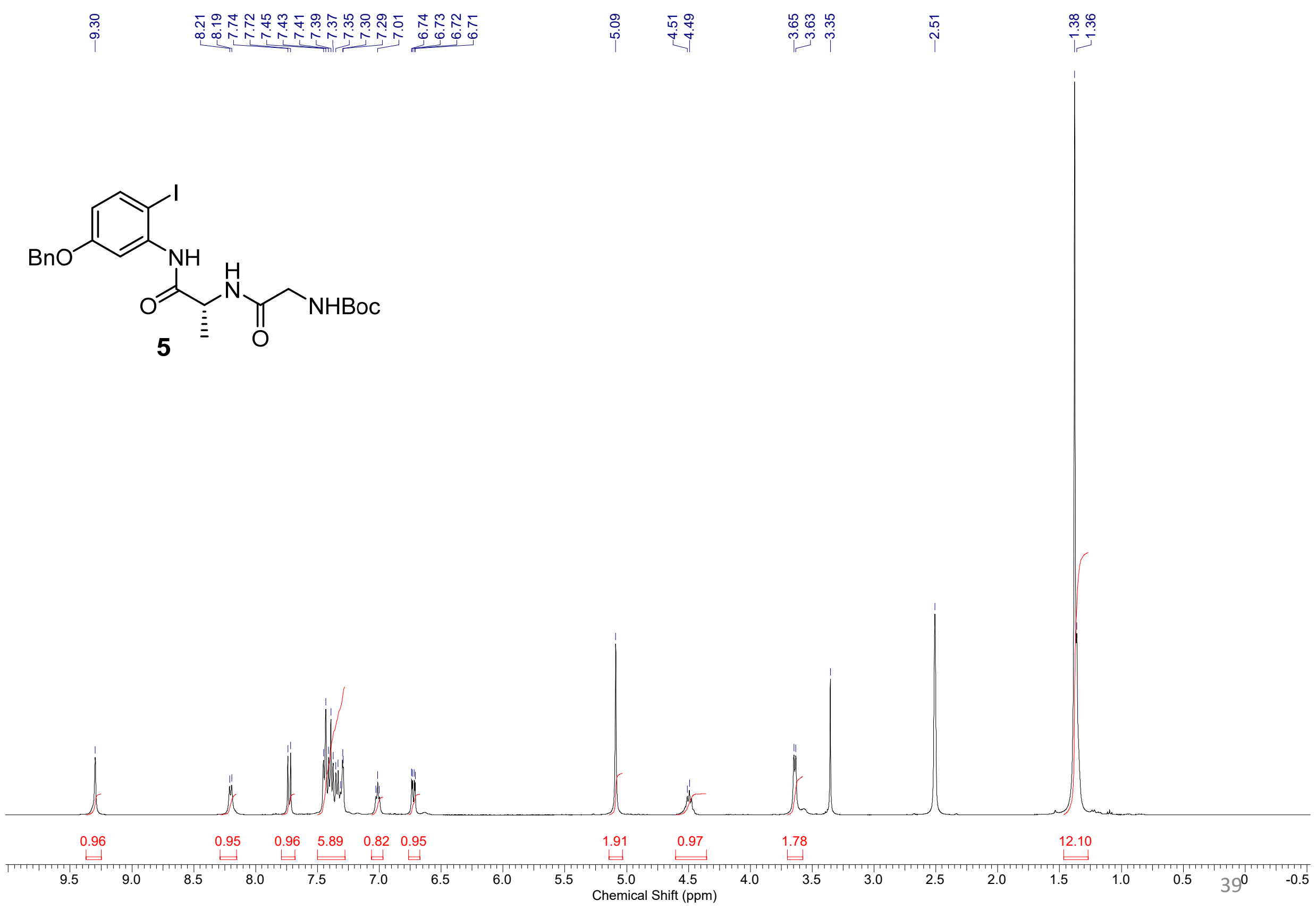




\section{${ }^{13} \mathrm{C}$ NMR (100 MHz, DMSO-d 6 ) of compound 5}

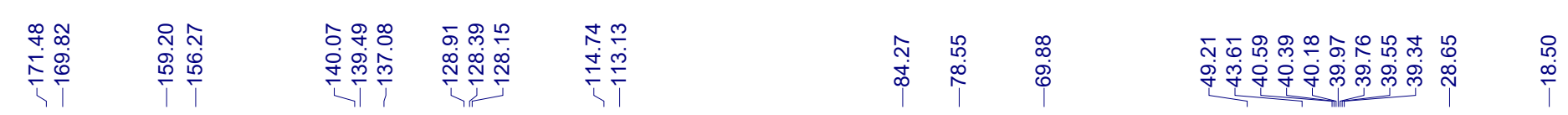<smiles>C[C@H](NC(=O)NCC(=O)OC(C)(C)C)C(=O)Nc1cc(OC(=O)c2ccccc2)ccc1I</smiles>

5 
${ }^{1} \mathrm{H}$ NMR (400 MHz, DMSO-d ${ }_{6}$ ) of compound 12

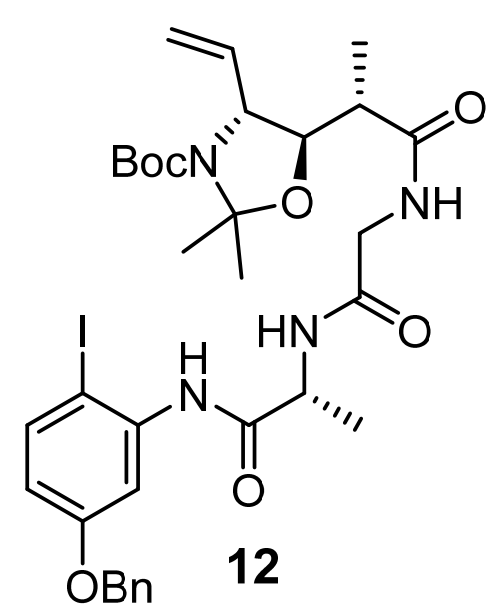




\section{${ }^{13} \mathrm{C}$ NMR (100 MHz, CD $\left.\mathrm{OD}\right)$ of compound 12}

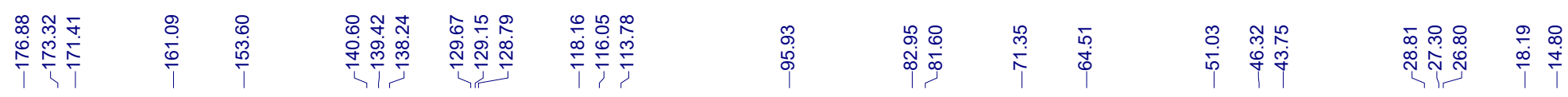<smiles>C=C[C@H]1[C@H]([C@@H](C)C(=O)NCC(=O)N[C@@H](C)C(=O)Nc2cc(OCc3ccccc3)ccc2I)OC(C)(C)N1C(=O)OCc1ccccc1</smiles>

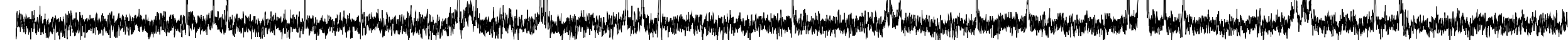

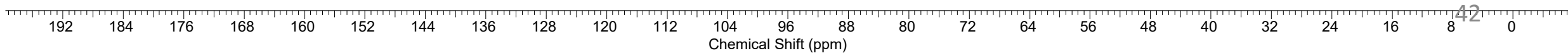


${ }^{1} \mathrm{H}$ NMR (400 MHz, $\left.\mathrm{CD}_{3} \mathrm{OD}\right)$ of compound 4

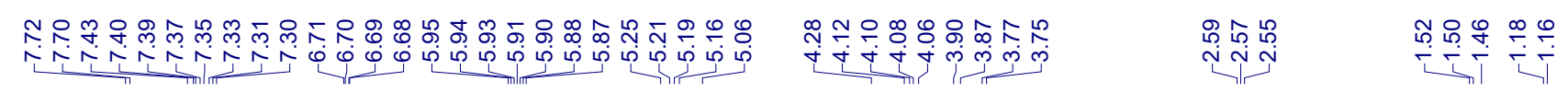
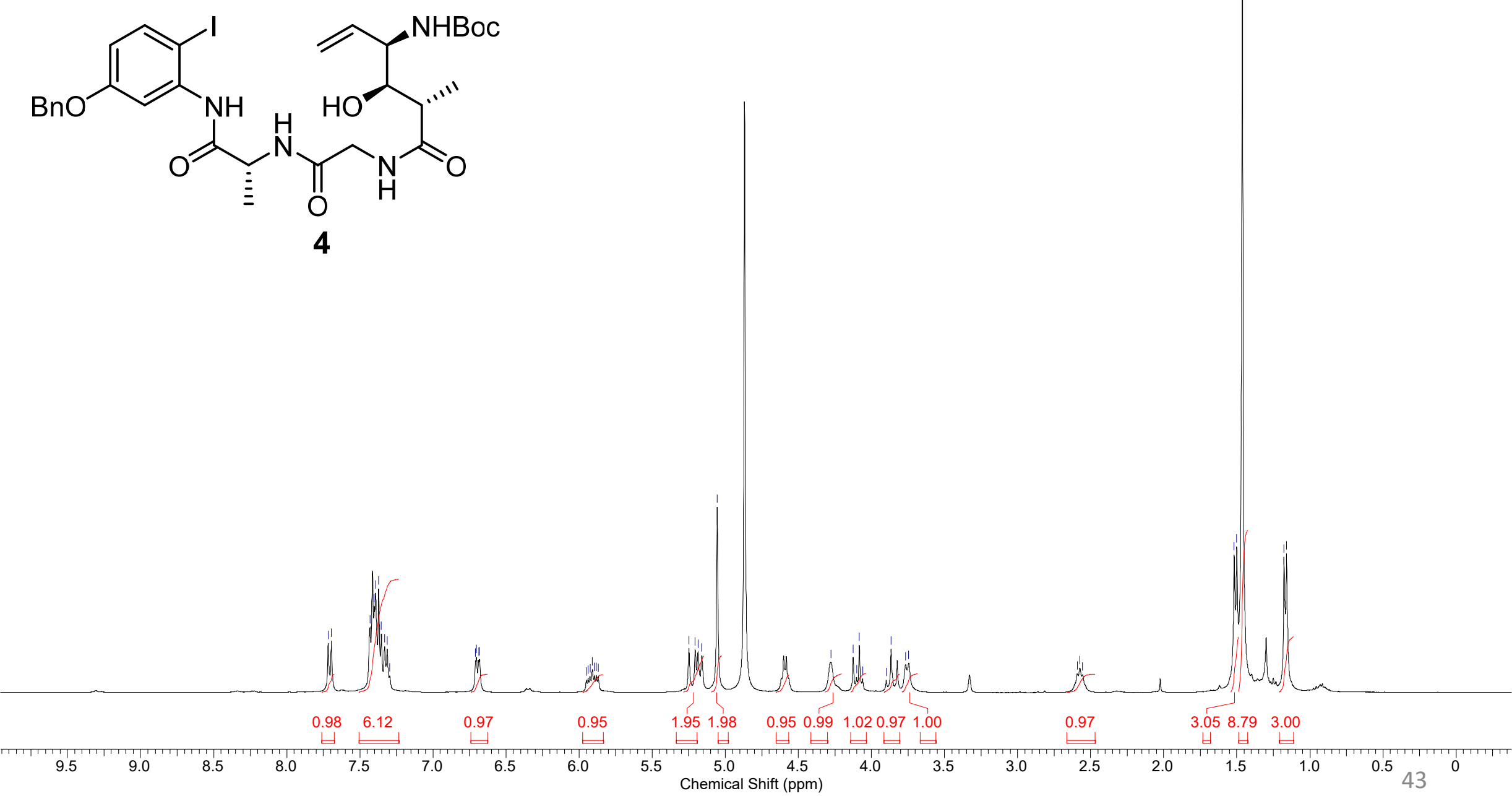


\section{${ }^{13} \mathrm{C}$ NMR (100 MHz, $\left.\mathrm{CD}_{3} \mathrm{OD}\right)$ of compound 4}

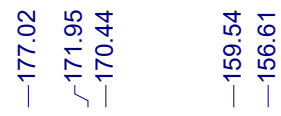

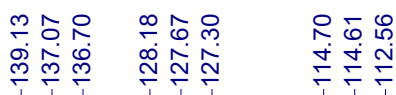

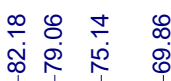

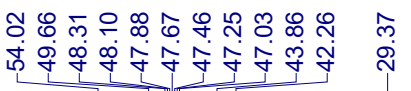
용요<smiles>C=CC(NCCC(C)(C)C)[C@H](C)[C@@H](O)C(=O)NCC(=O)N[C@@H](C)C(=O)Nc1cc(OCc2ccccc2)ccc1I</smiles>

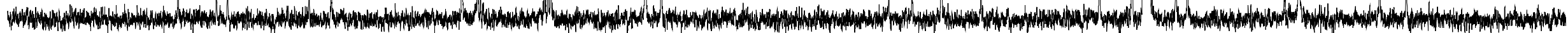

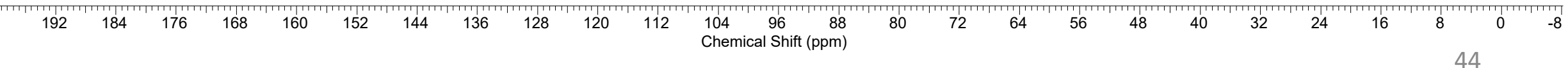




\section{${ }^{1} \mathrm{H}$ NMR (400 MHz, $\left.\mathrm{CD}_{\mathbf{3}} \mathrm{OD}\right)$ of compound 3}

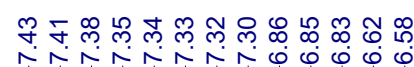

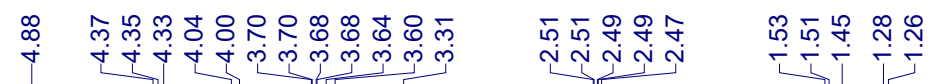

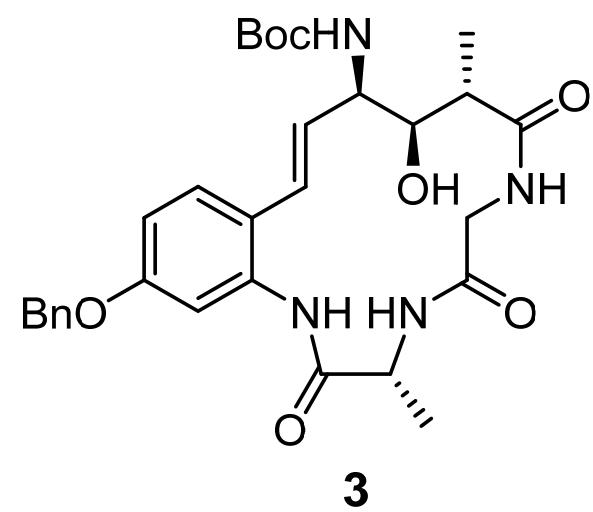

$2.273 .12 \quad 1.040 .840 .830 .78$

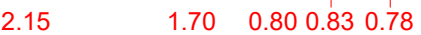

0.88

3.009 .042 .89 


\section{${ }^{13} \mathrm{C}$ NMR (100 MHz, $\left.\mathrm{CD}_{3} \mathrm{OD}\right)$ of compound 3}

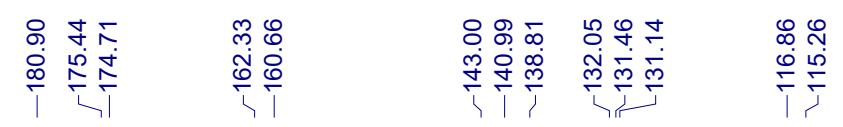

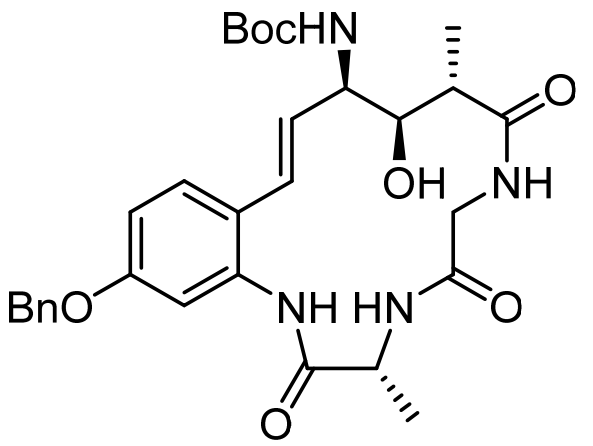

3

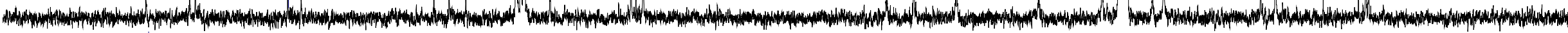

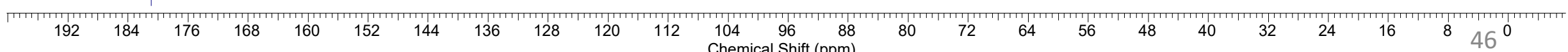


${ }^{1}$ H NMR (500 MHz, DMSO-d ${ }_{6}$ ) of compound 14

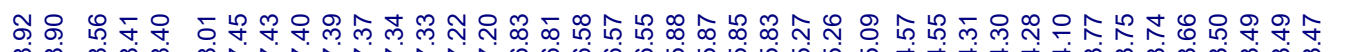

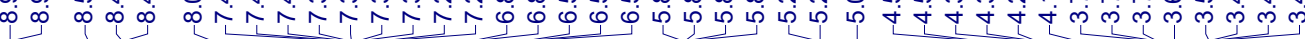

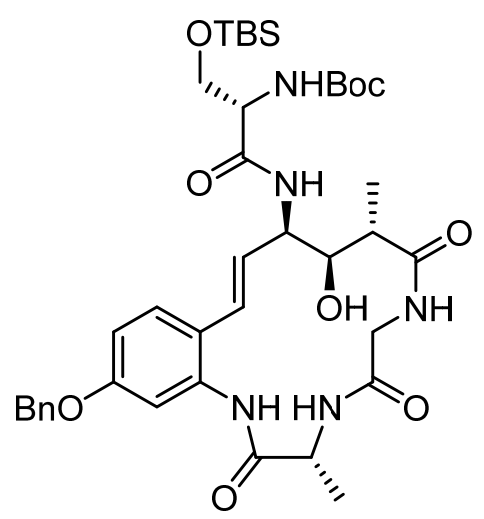

14

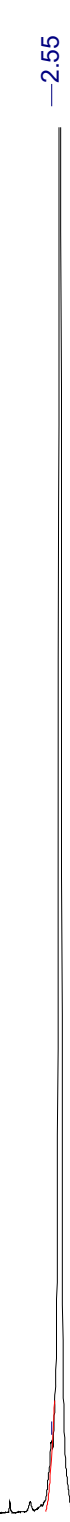

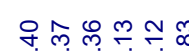

ชั่

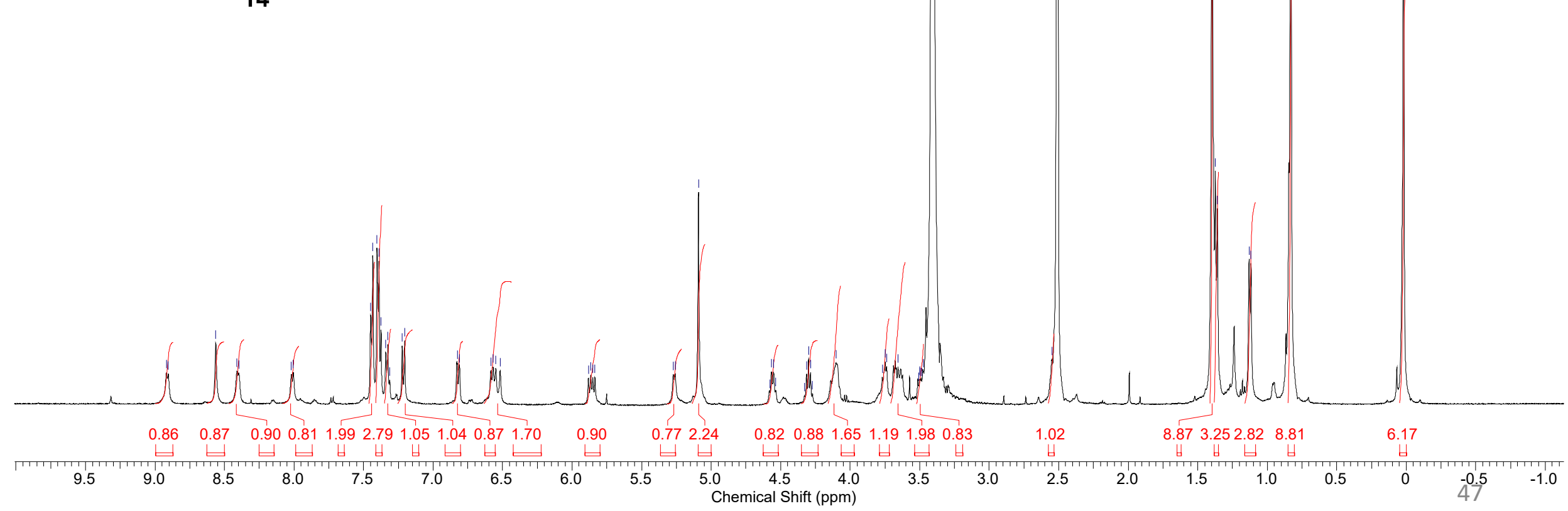


${ }^{13} \mathrm{C}$ NMR (125 MHz, DMSO-d ${ }_{6}$ ) of compound 14

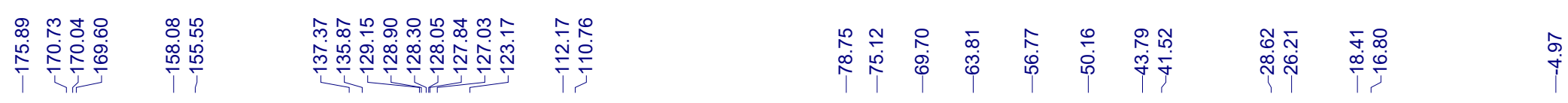

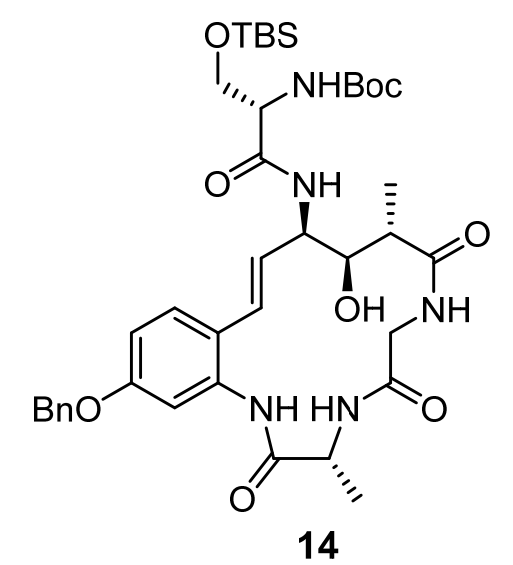


${ }^{1}$ H NMR (400 MHz, DMSO-d ${ }_{6}$ ) of compound 15

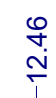

ㅌㅇㅇ

の̆

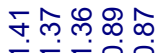

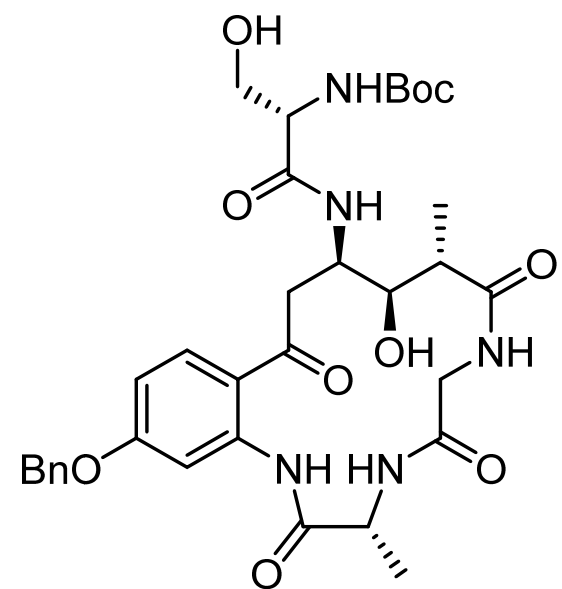

15

0.91

$0.890 .890 .921 .002 .012 .251 .181 .000 .730 .90 \quad 0.861 .841 .840 .860 .900 .912 .922 .030 .830 .92$

8.863 .172 .94 
${ }^{13} \mathrm{C}$ NMR (125 MHz, DMSO-d ${ }_{6}$ ) of compound 15

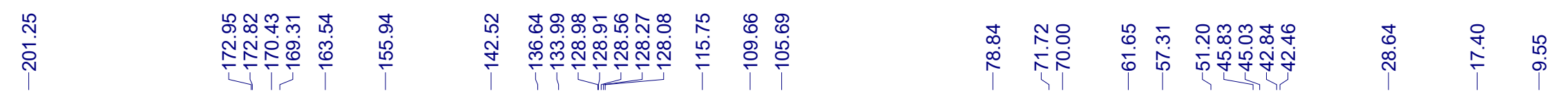<smiles>CC(=O)OCCCCNC(=O)[C@H](CO)NC(=O)C[C@@H](O)[C@H](C)C(=O)NCC(=O)N[C@@H](C)C(=O)Nc1cccc(OCc2ccccc2)c1</smiles>

15

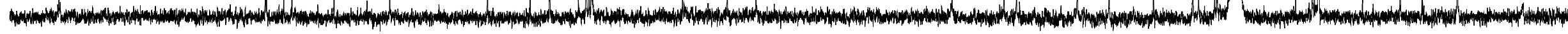

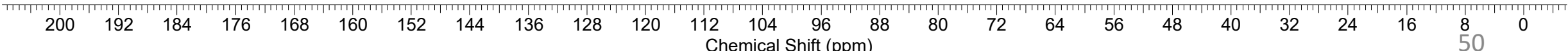




\section{${ }^{1}$ H NMR (400 MHz, DMSO-d ${ }_{6}$ ) of compound 17}

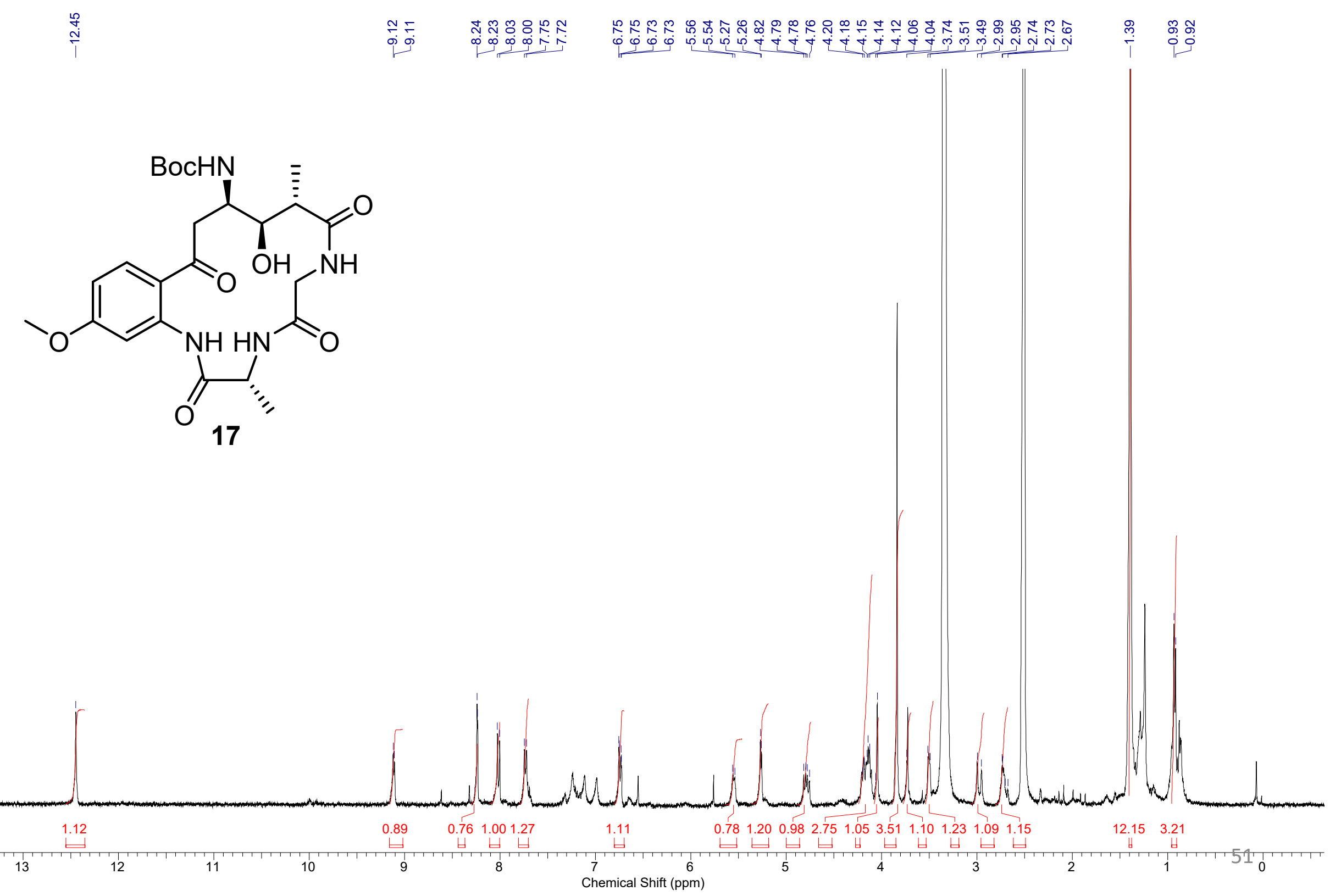


${ }^{13} \mathrm{C}$ NMR (100 MHz, DMSO-d ${ }_{6}$ of compound 17
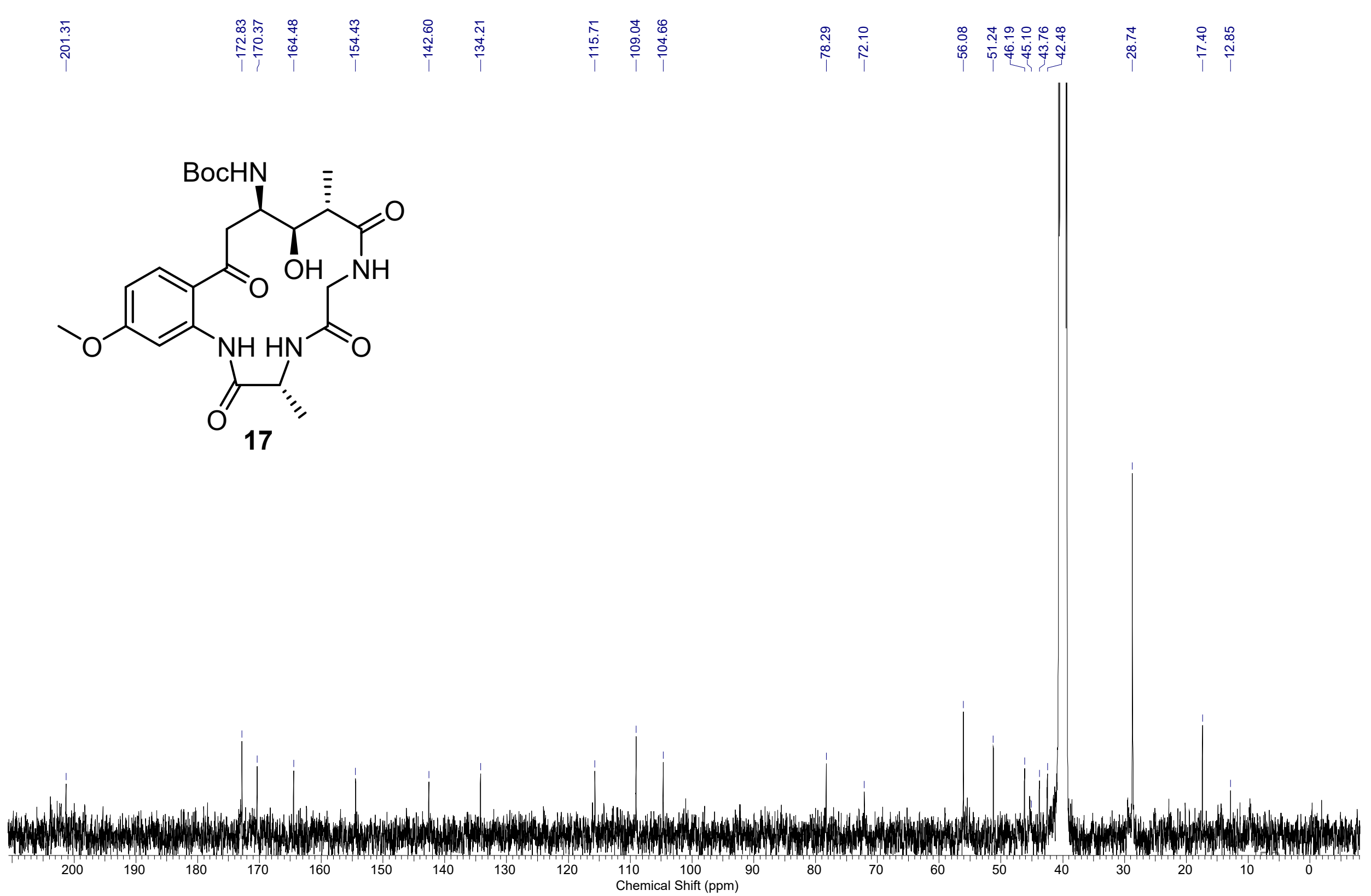
${ }^{1} \mathrm{H}$ NMR (400 MHz, CD $\left.\mathrm{CD}_{3} \mathrm{OD}\right)$ of compound 18

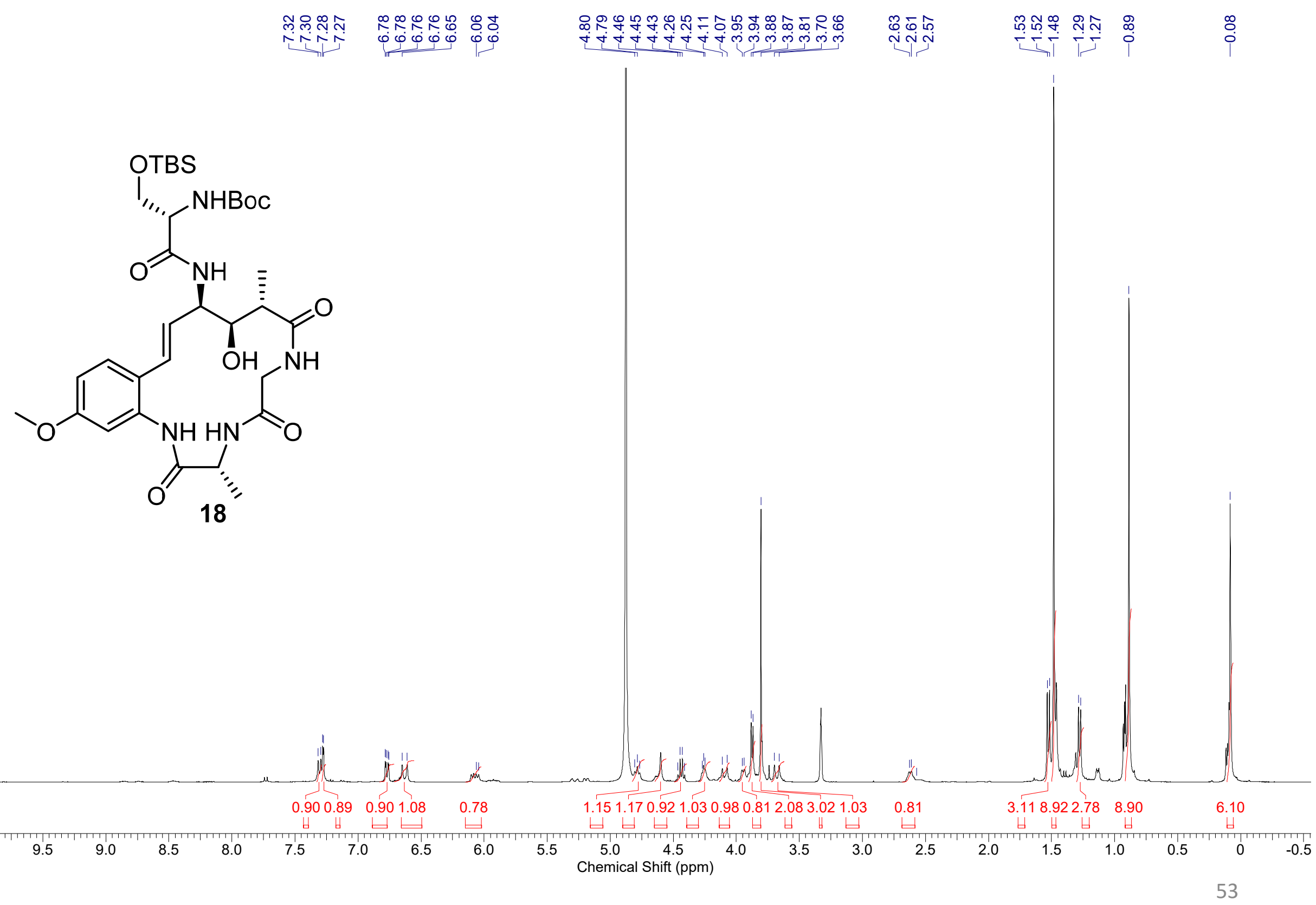


${ }^{13}$ C NMR (125 MHz, DMSO-d $)$ of compound 18

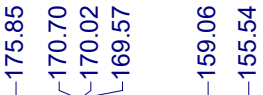

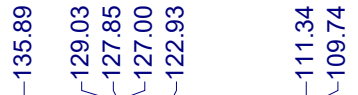
$\underset{\substack{1 \\ \infty}}{\infty}$

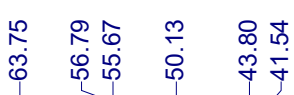

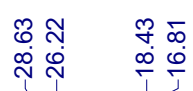

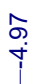

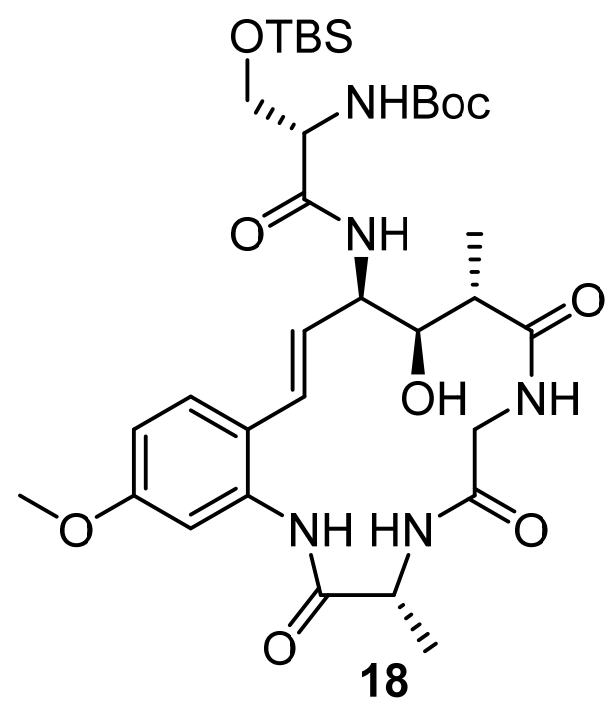

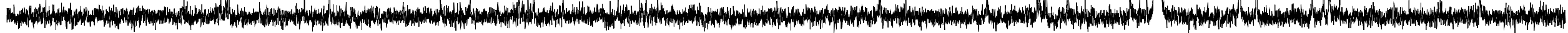

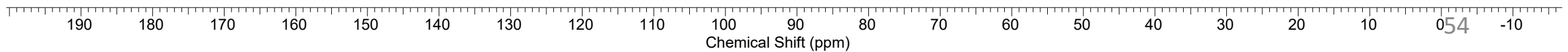


${ }^{1}$ H NMR (400 MHz, DMSO-d $)_{6}$ of compound 19

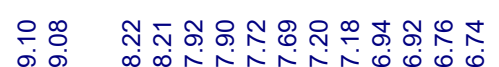

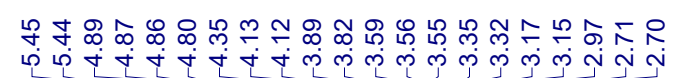

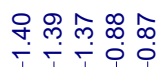

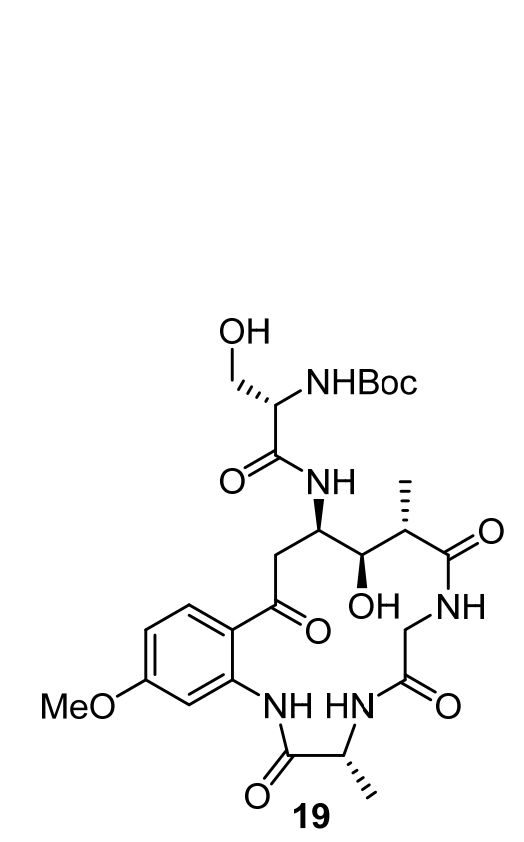

a montritiogo

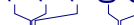

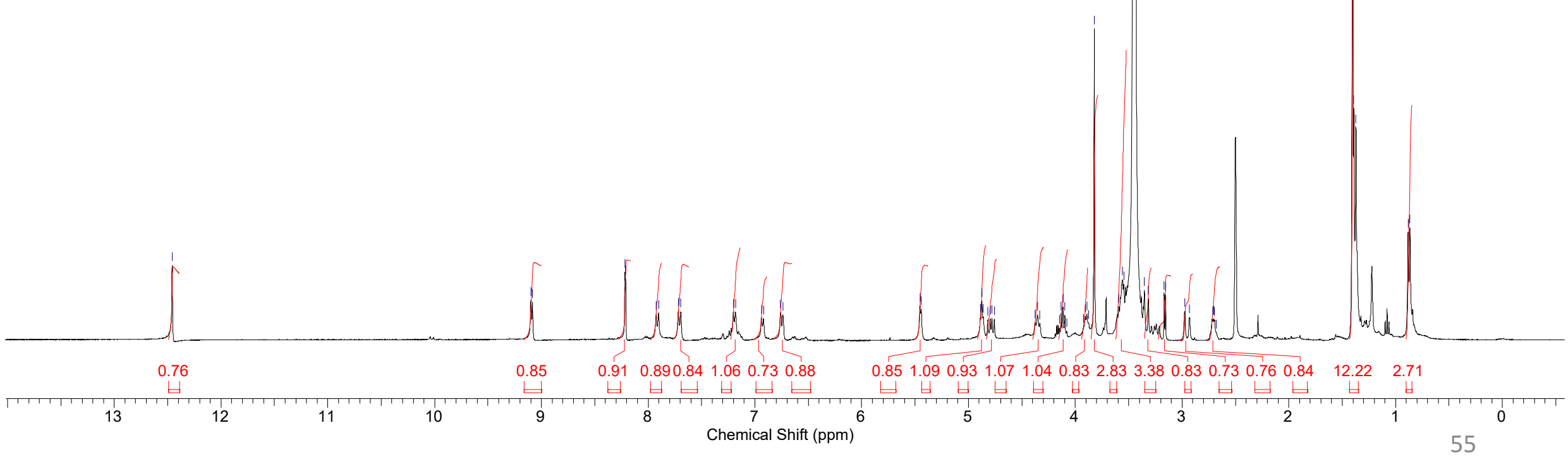


${ }^{13} \mathrm{C}$ NMR (100 MHz, DMSO-d 6 ) of compound 19

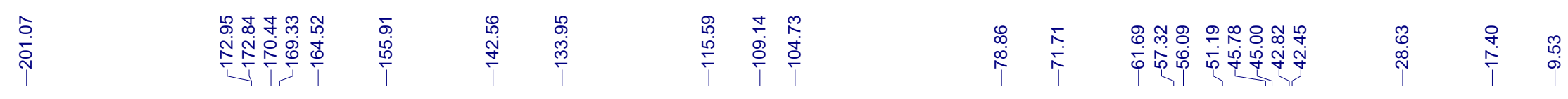<smiles>COc1ccc(C(=O)CC(NC(=O)C(CO)NC(=O)OC(C)(C)C)C(O)[C@H](C)C(=O)NCC(=O)N[C@@H](C)C(=O)Nc2cc(OC)ccc2C)c(C)c1</smiles> 
${ }^{1}$ H NMR (500 MHz, DMSO-d 6 ) of compound 2

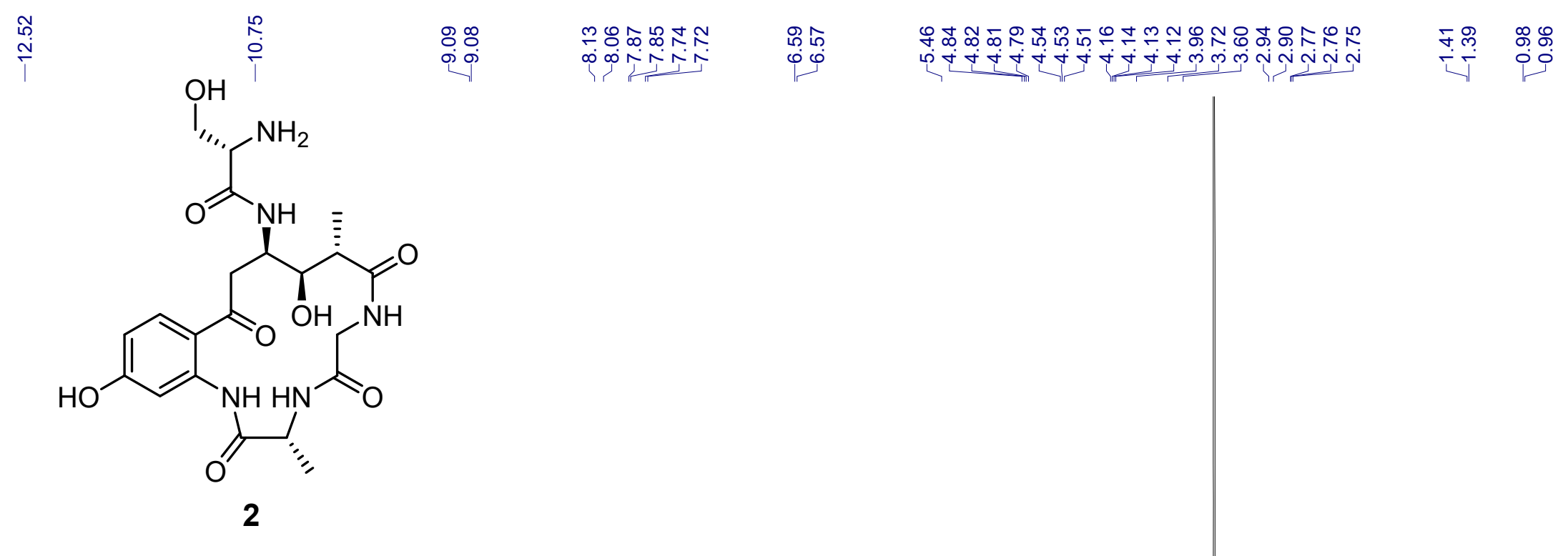

toluene

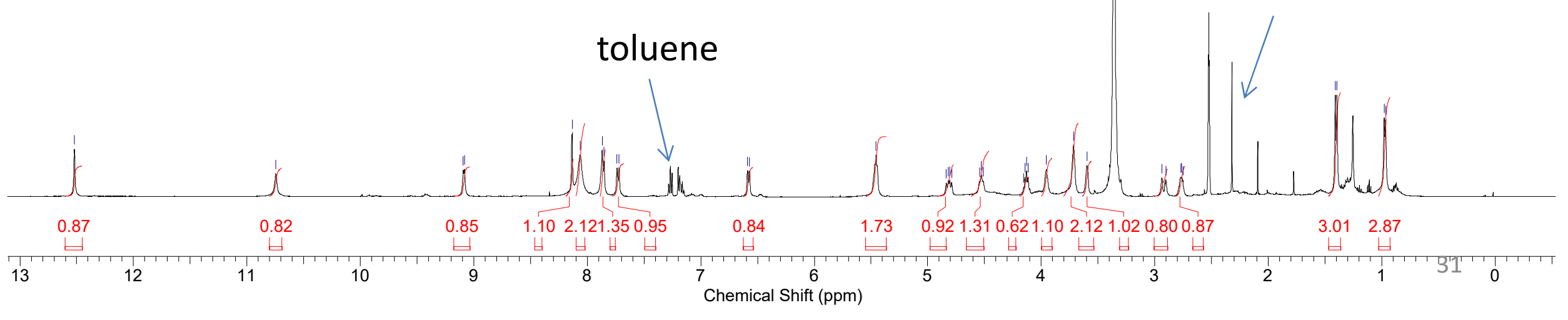




\section{${ }^{13}$ C NMR (125 MHz, DMSO-d 6 ) of compound 2}

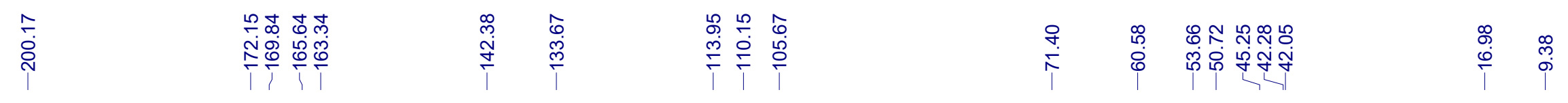<smiles>[Z]C(NC(=O)CNC(=O)[C@H](C)[C@H](O)[C@H](CC(=O)c1ccc(O)cc1NC(=O)[C@H](N)CO)NC(=O)[C@H](C)CO)C(=O)N[C@@H](C)CO</smiles>

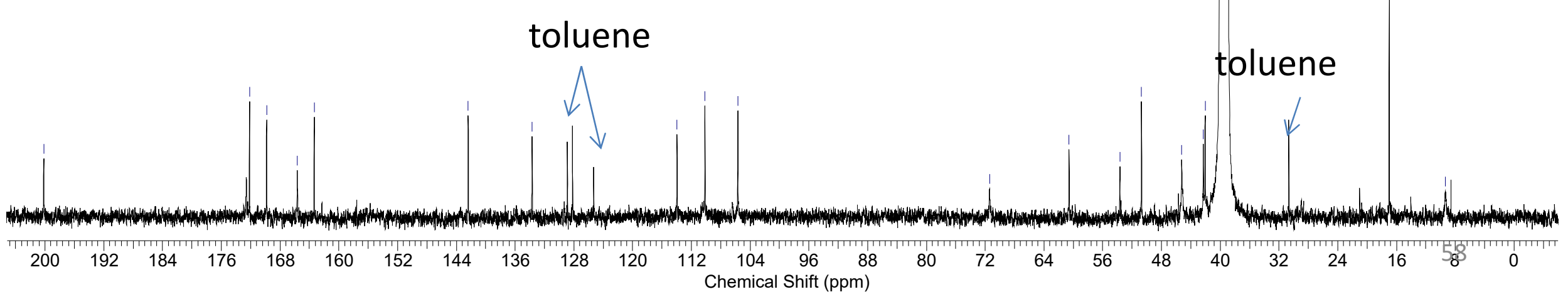


HMBC spectrum (500 MHz, DMSO-d $)_{6}$ of compound 2

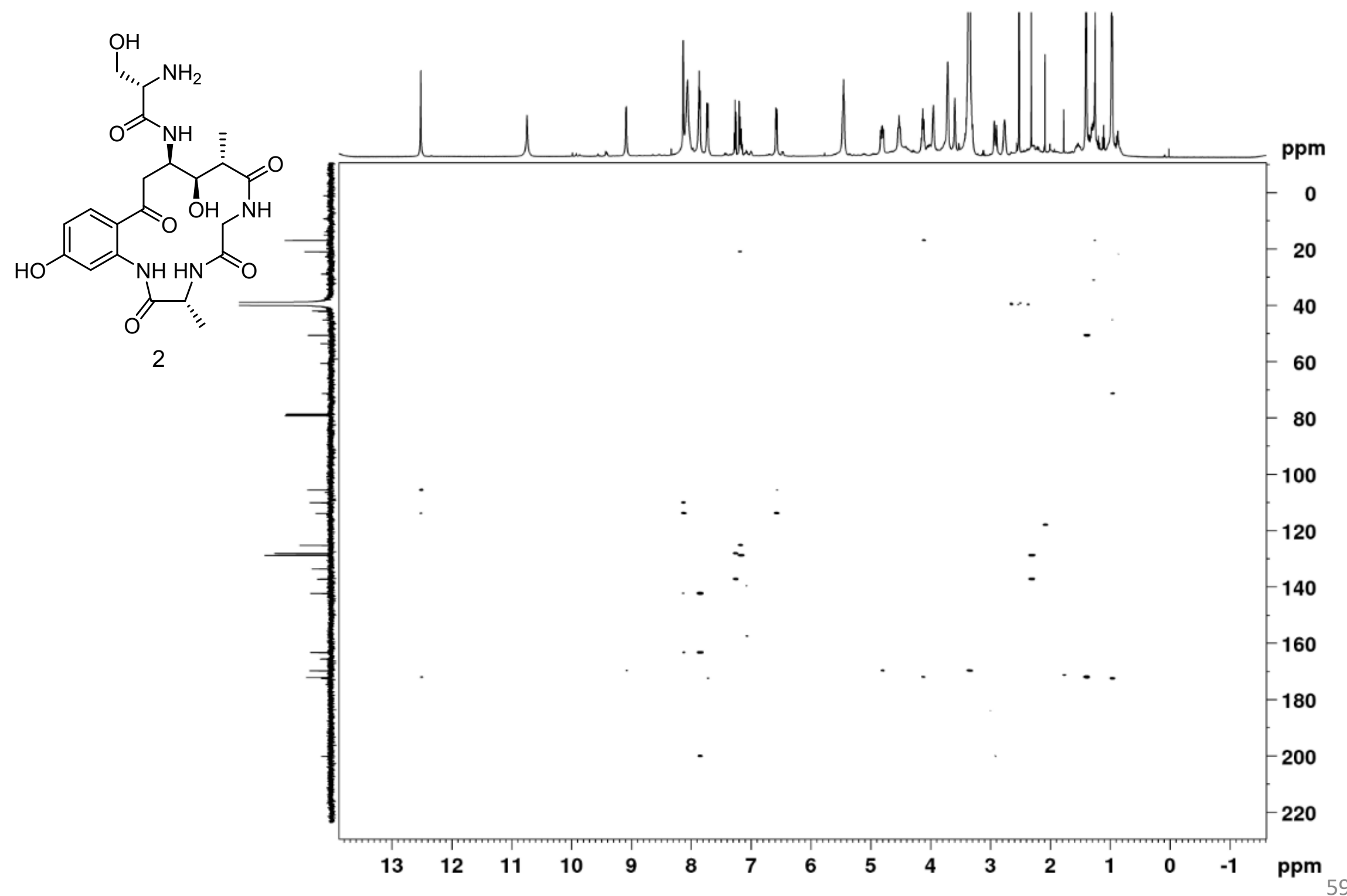


HSQC spectrum (500 MHz, DMSO-d 6 ) of compound 2

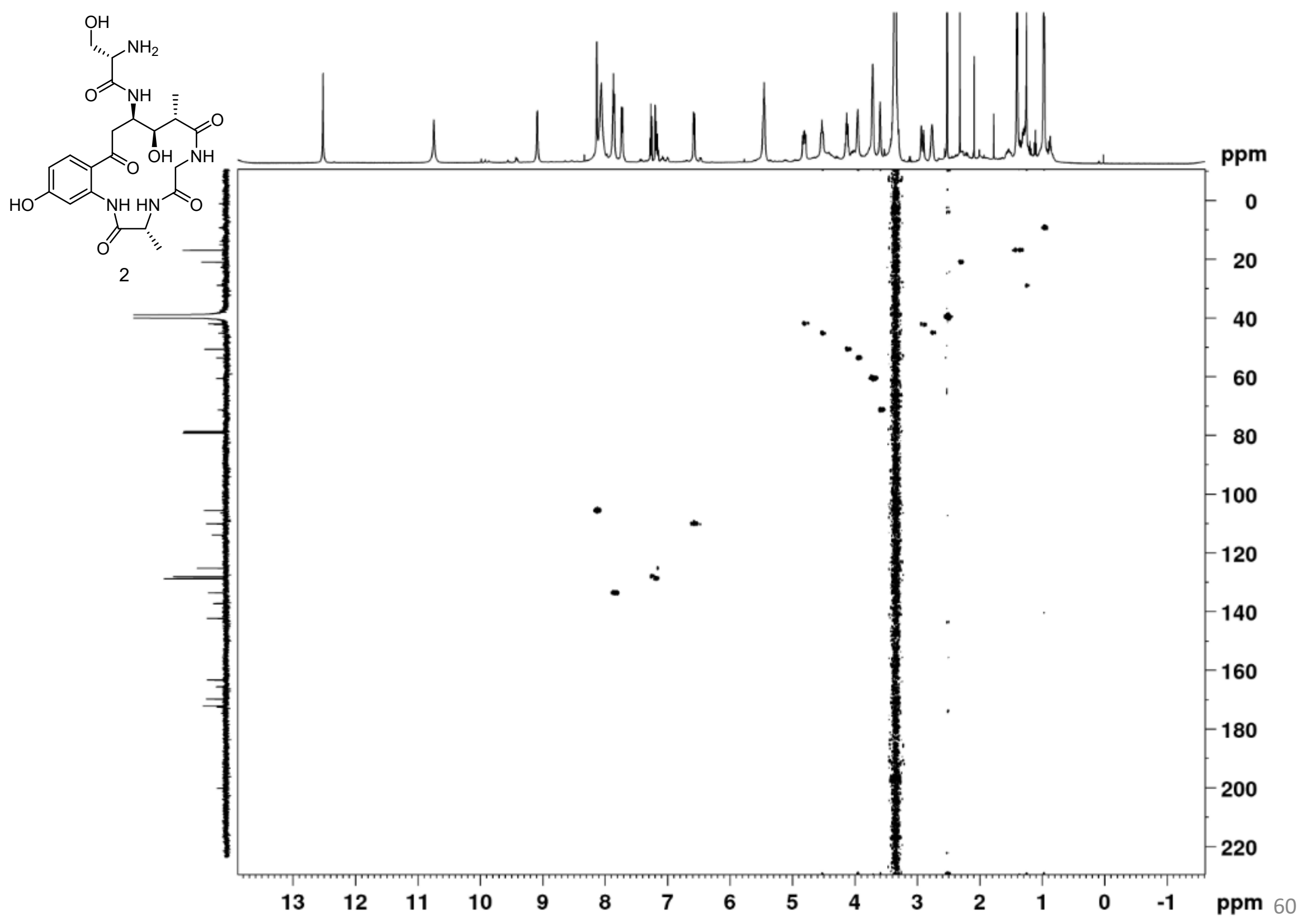


ROESY spectrum (500 MHz, DMSO-d ${ }_{6}$ ) of compound 2

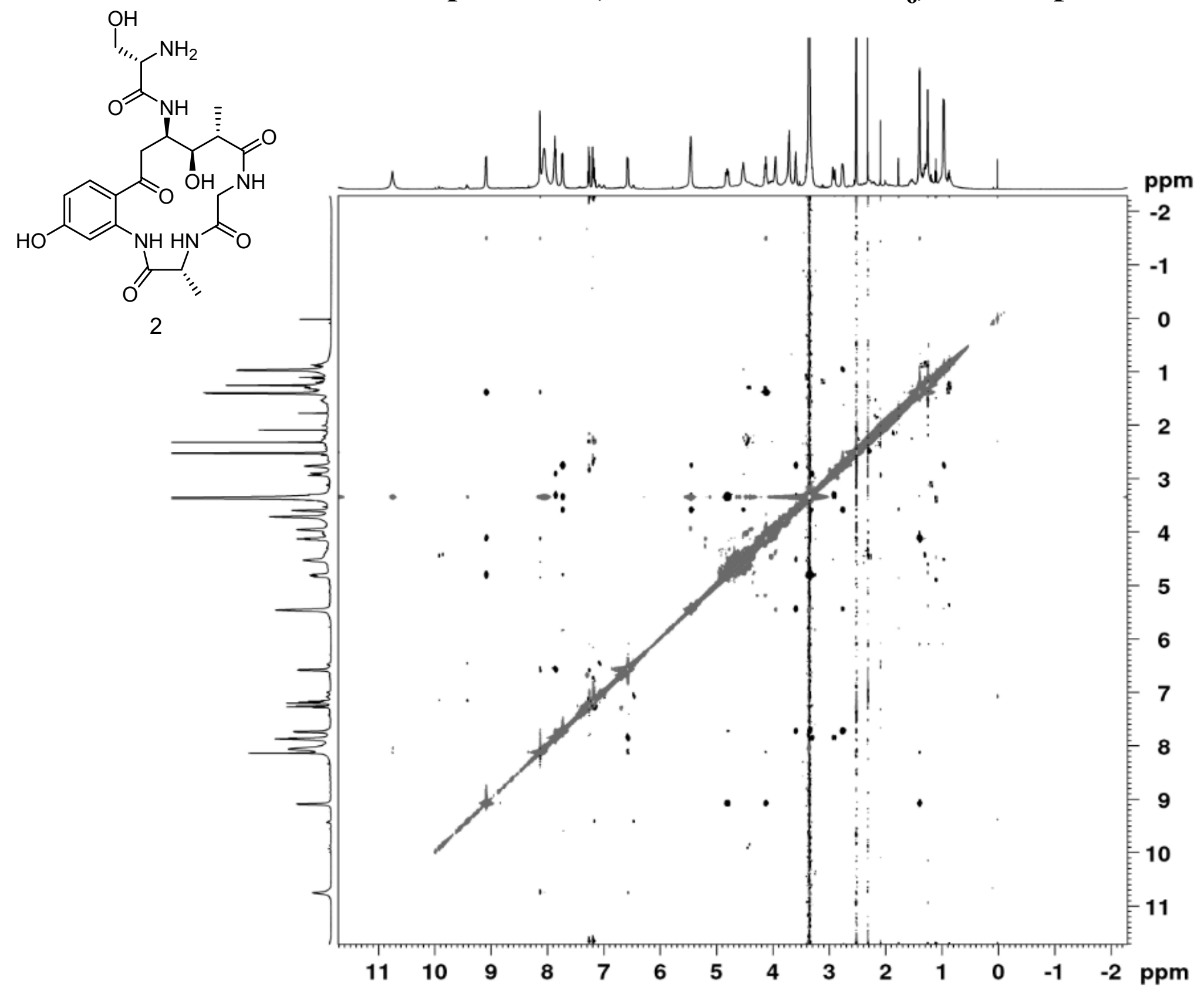


TOCSY spectrum (500 MHz, DMSO-d ${ }_{6}$ ) of compound 2

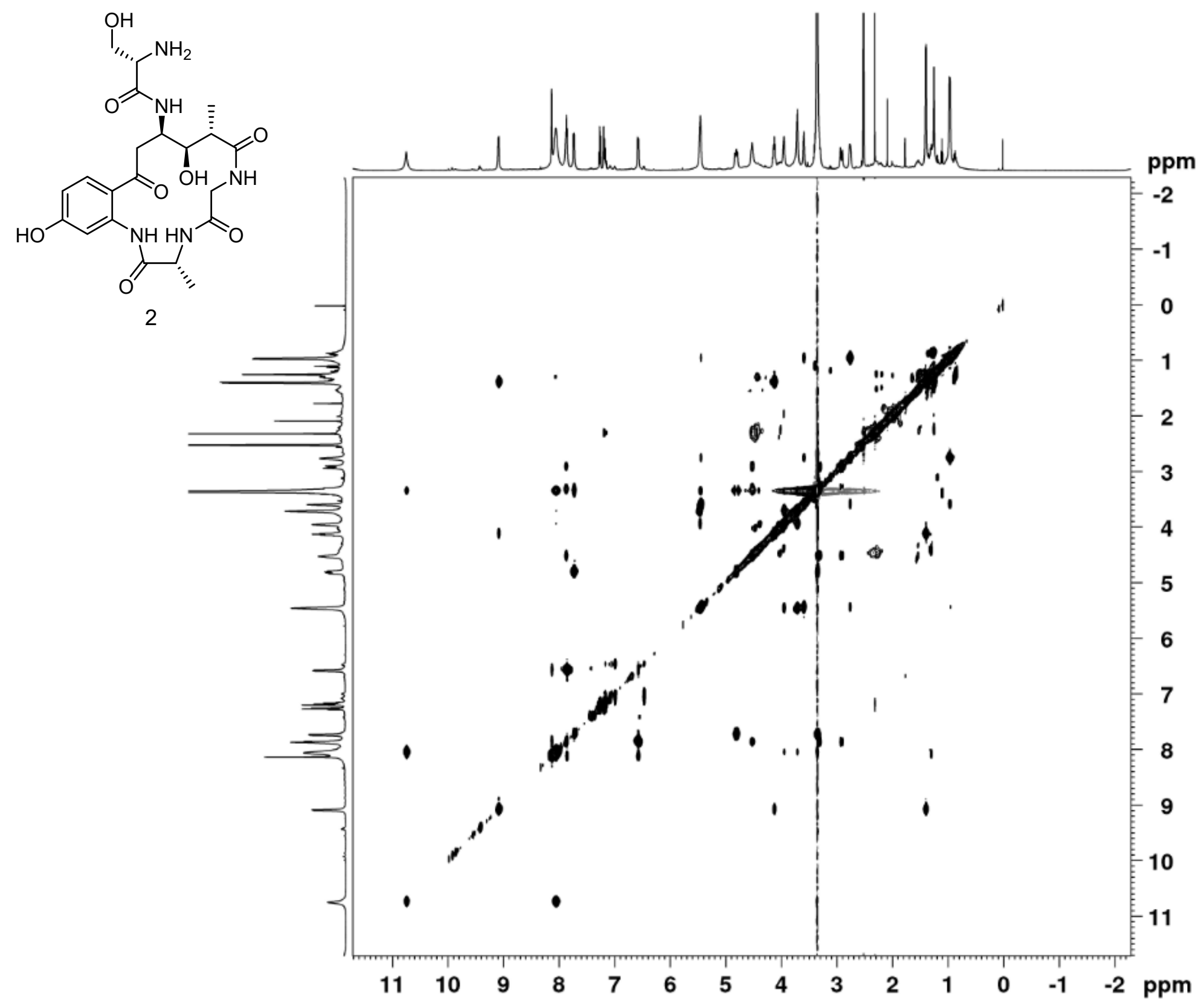




\section{DQF-COSY spectrum (500 MHz, DMSO-d $\left.{ }_{6}\right)$ of compound 2}

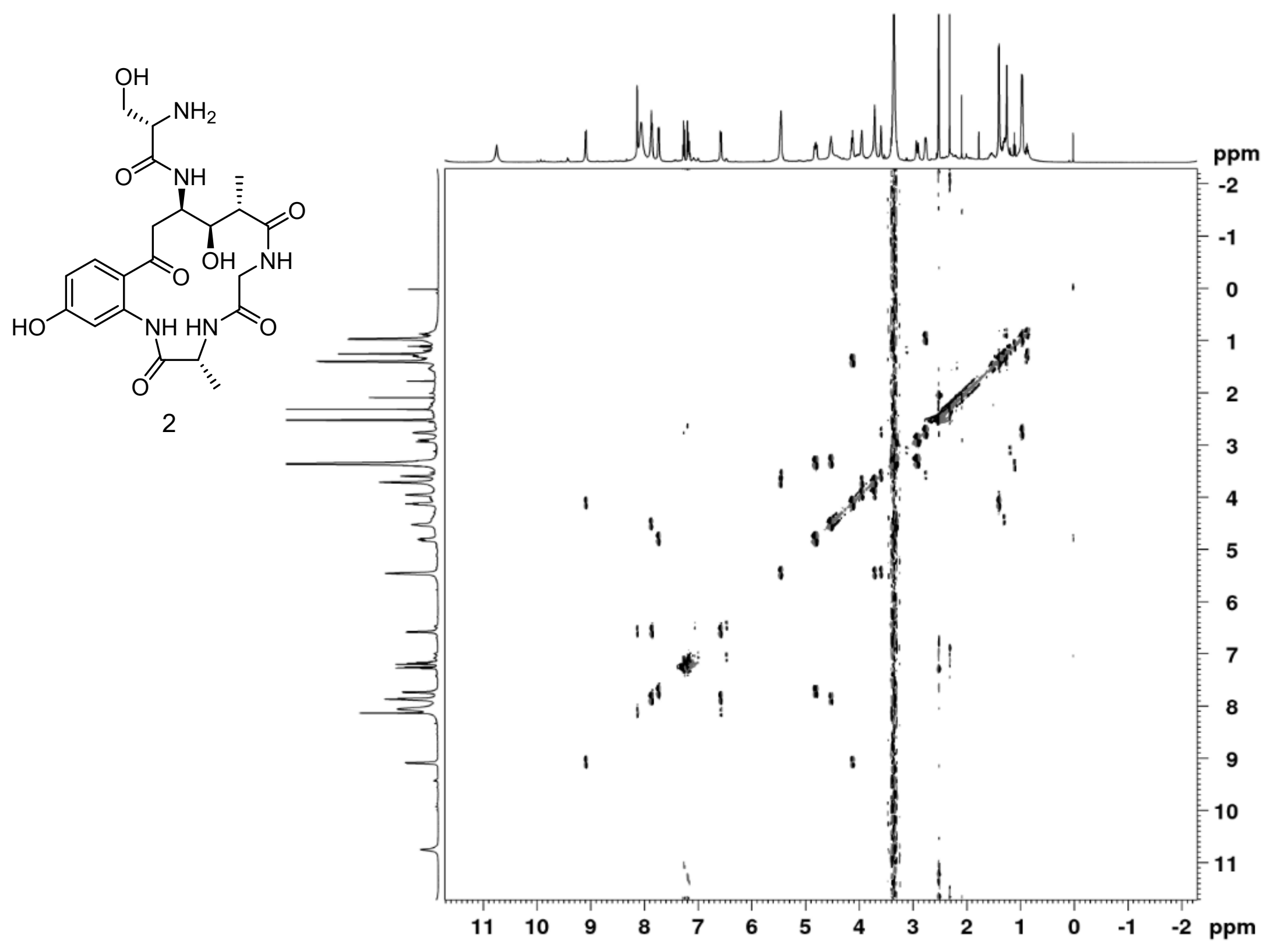


${ }^{1}$ H NMR (500 MHz, DMSO-d ${ }_{6}$ ) of compound 23

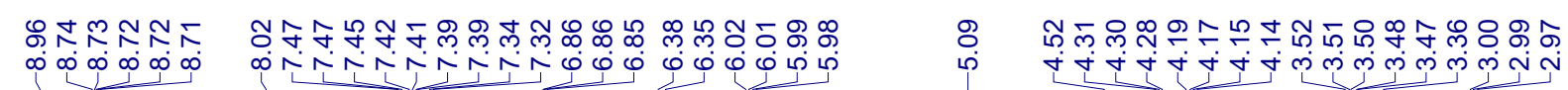

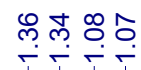<smiles>C[C@@H]1NC(=O)CNC(=O)[C@H](C)[C@H]2OC[C@H](/C=C/c3ccc(OCc4ccccc4)cc3NC2=O)NC(=O)NC1=O</smiles>

23

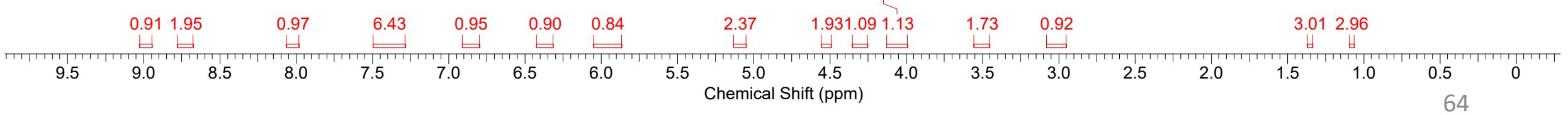




\section{${ }^{1} \mathrm{H}$ NMR (500 MHz, Acetone-d 6 ) of compound 23}

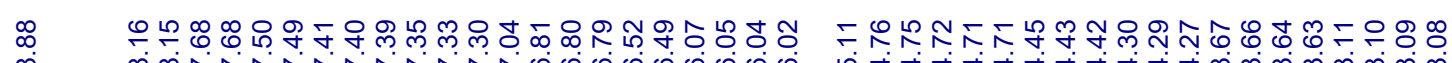

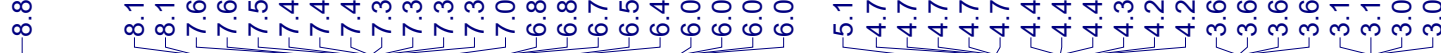

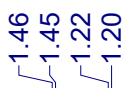

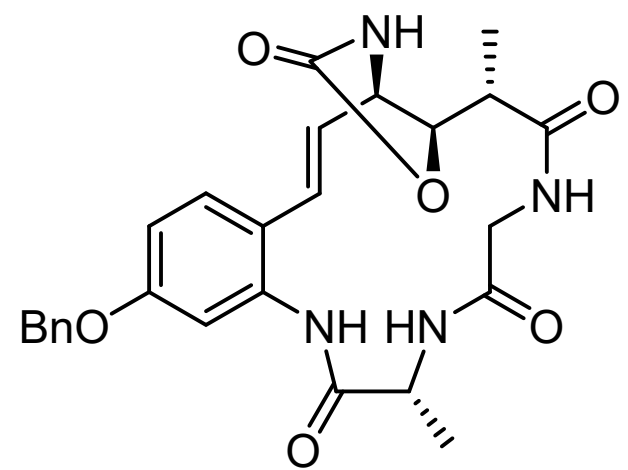

23 
HMBC spectrum (500 MHz, Acetone-d $)_{6}$ of compound 23

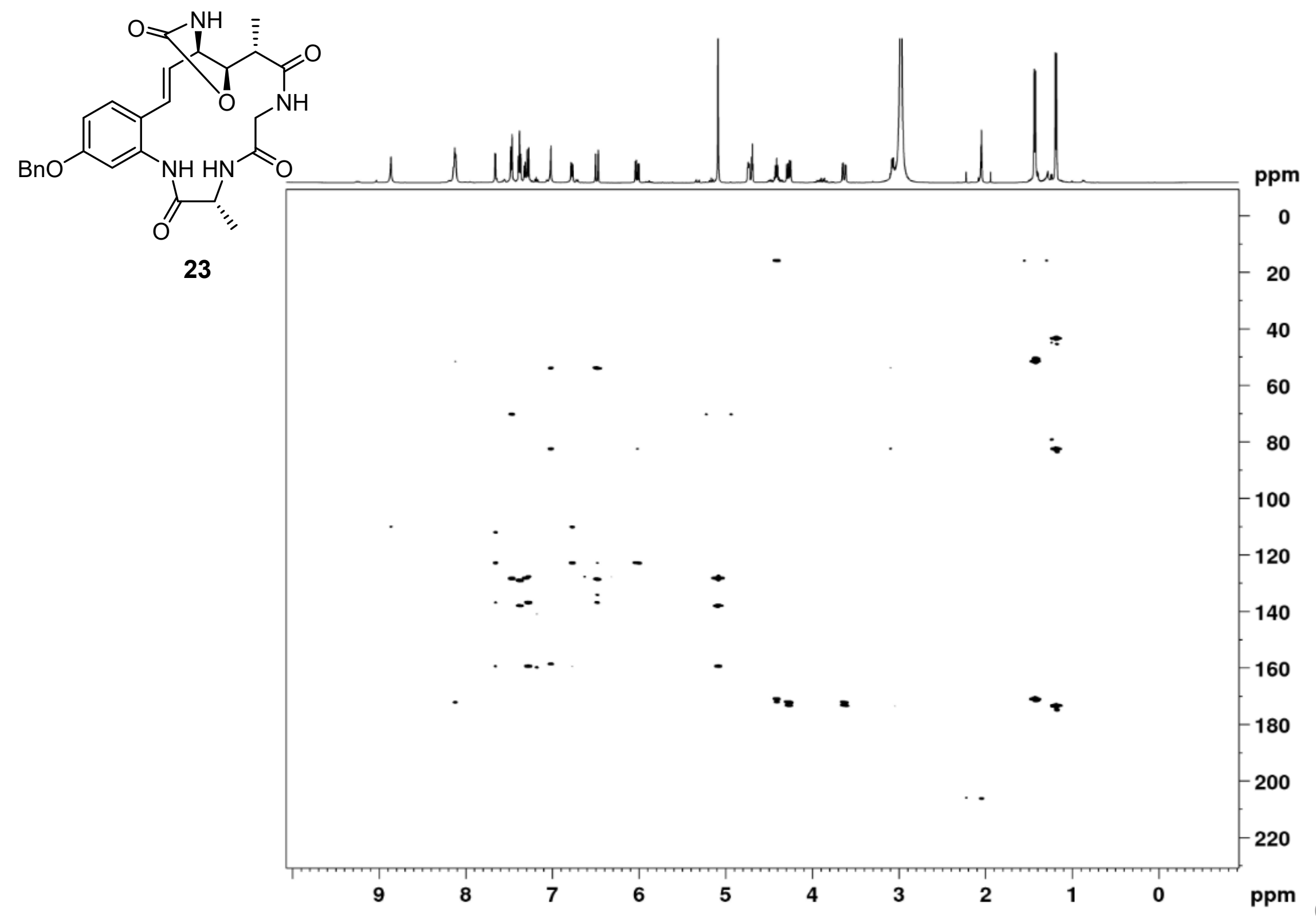


HSQC spectrum (500 MHz, Acetone-d ${ }_{6}$ ) of compound 23

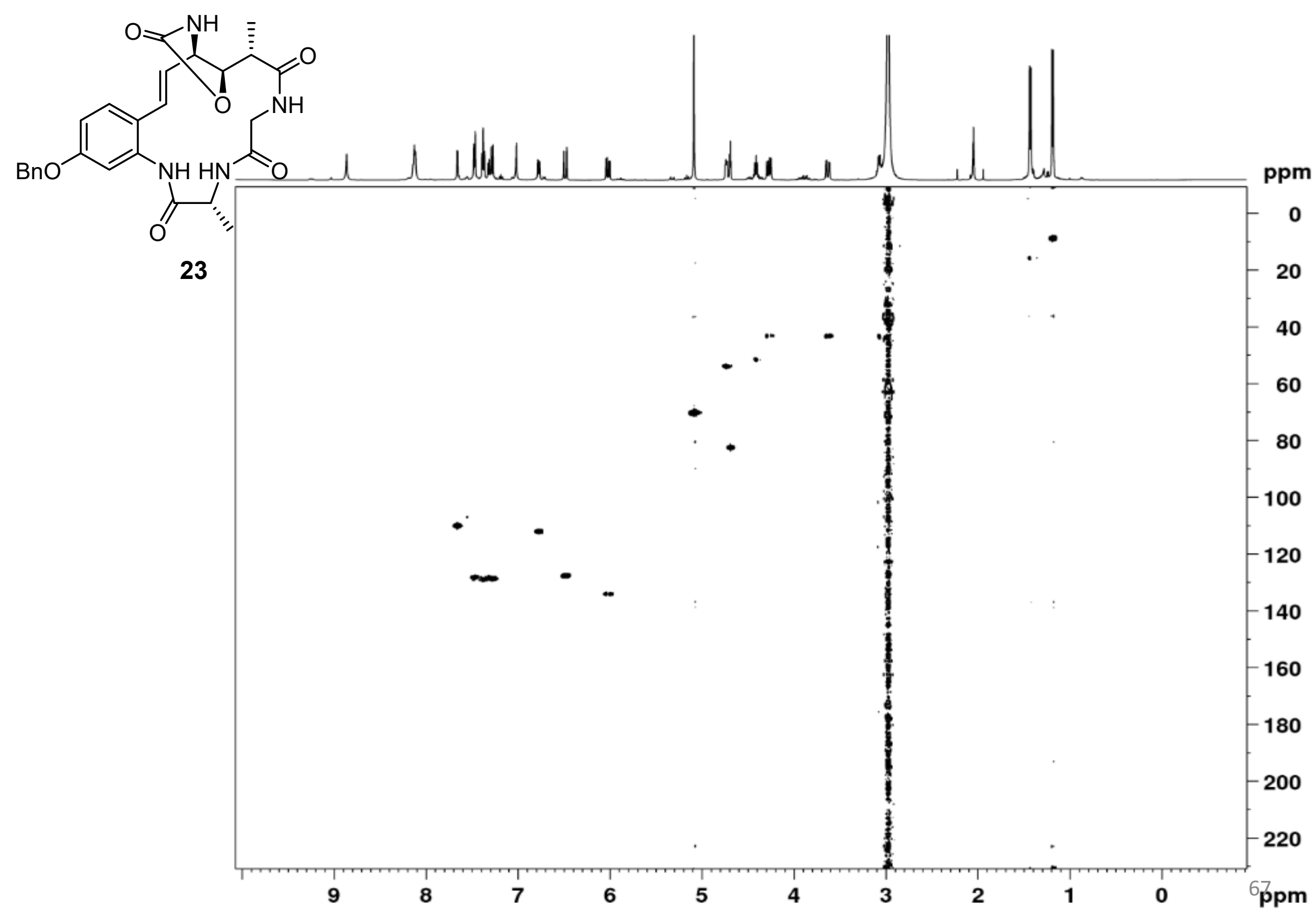


ROESY spectrum (500 MHz, Acetone-d $)_{6}$ of compound 23

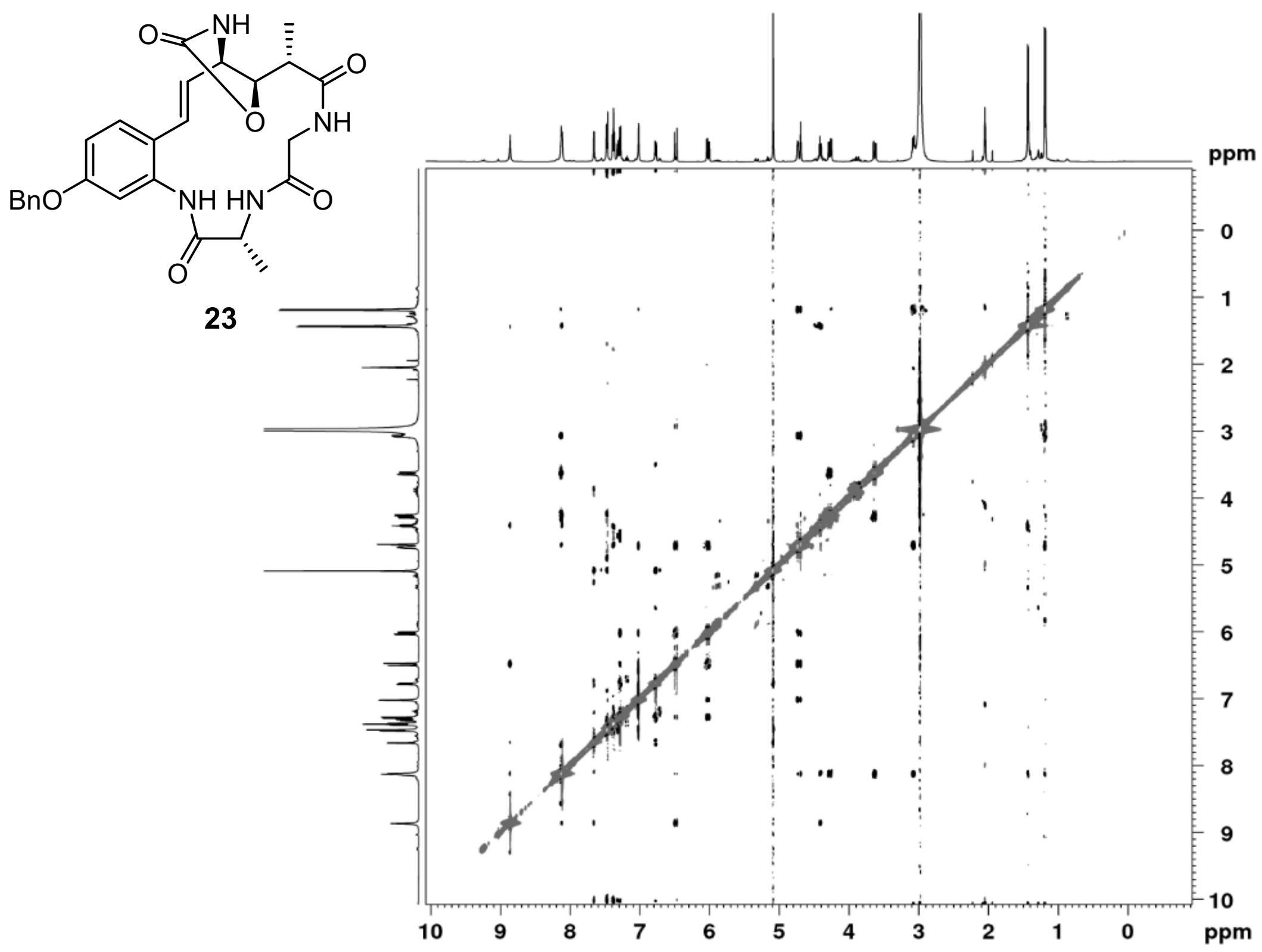


TOCSY spectrum (500 MHz, Acetone-d ${ }_{6}$ ) of compound 23

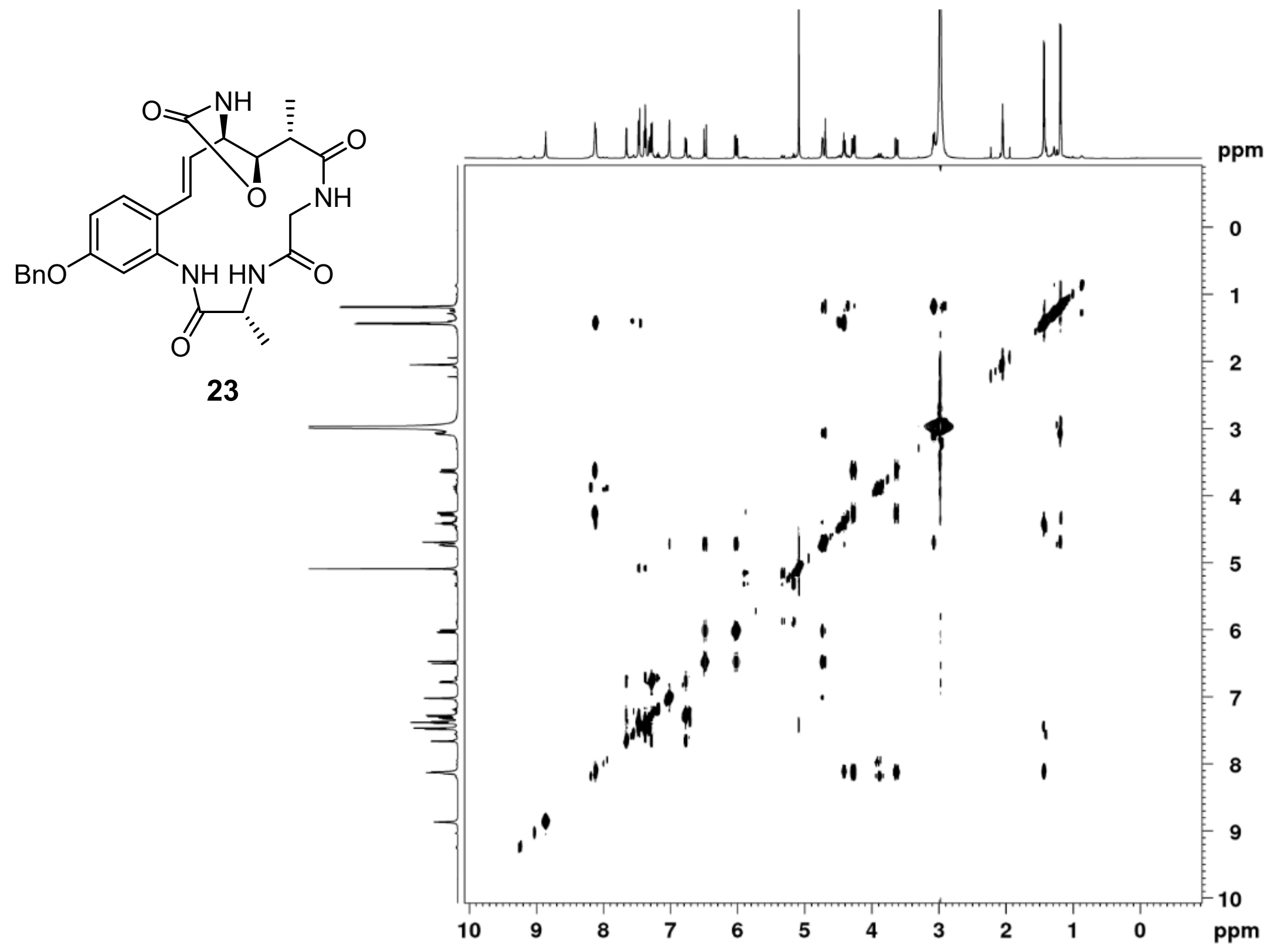


DQF-COSY spectrum (500 MHz, Acetone-d ${ }_{6}$ ) of compound 23

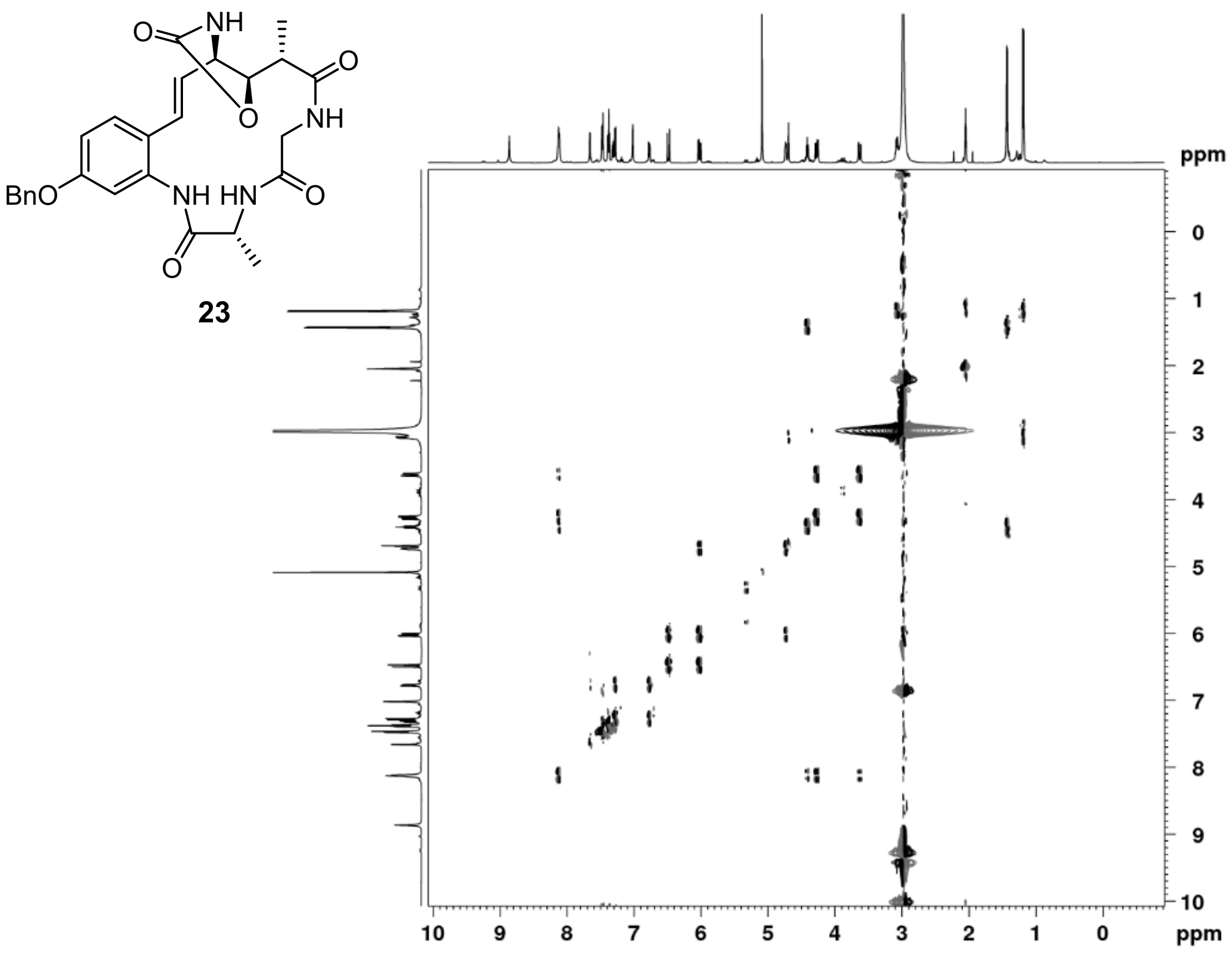




\section{${ }^{1} \mathrm{H}$ NMR (400 MHz, $\mathrm{CDCl}_{3}$ ) of compound 26}

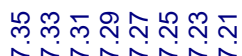

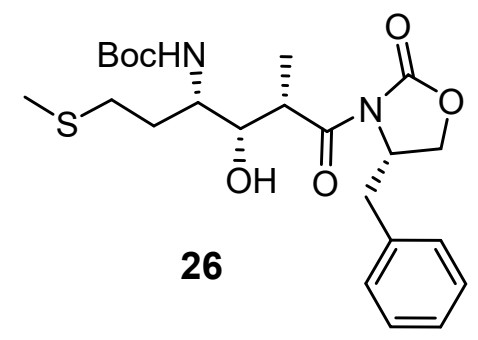

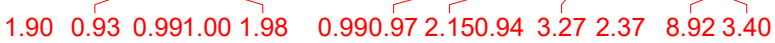

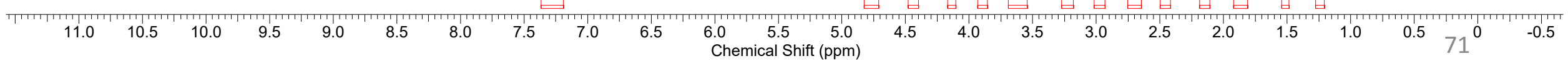


${ }^{13} \mathrm{C}$ NMR (100 MHz, $\left.\mathrm{CDCl}_{3}\right)$ of compound 26

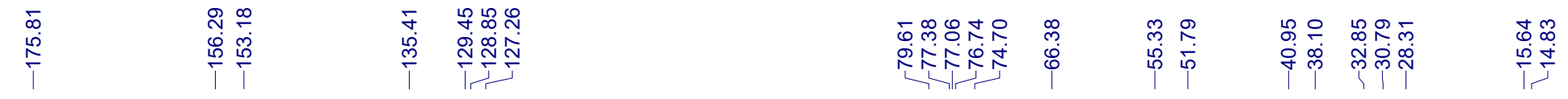

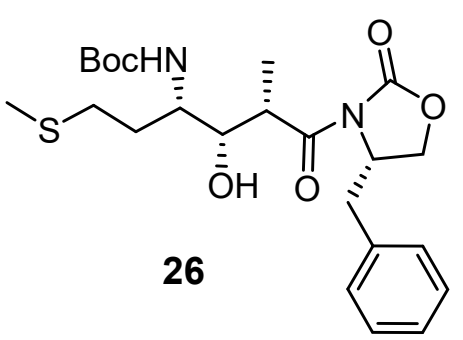

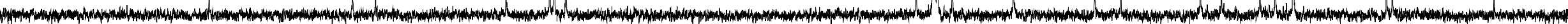

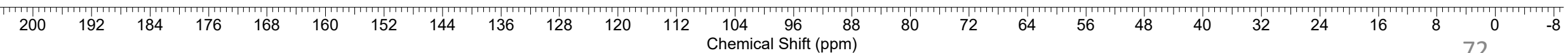
Chemical Shift (ppm) 


\section{${ }^{1} \mathrm{H}$ NMR (400 MHz, $\mathrm{CDCl}_{3}$ ) of compound 26'}

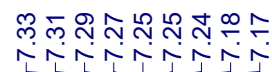

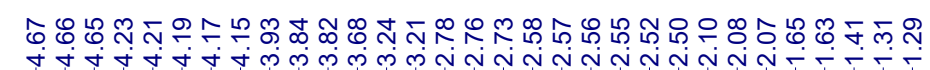
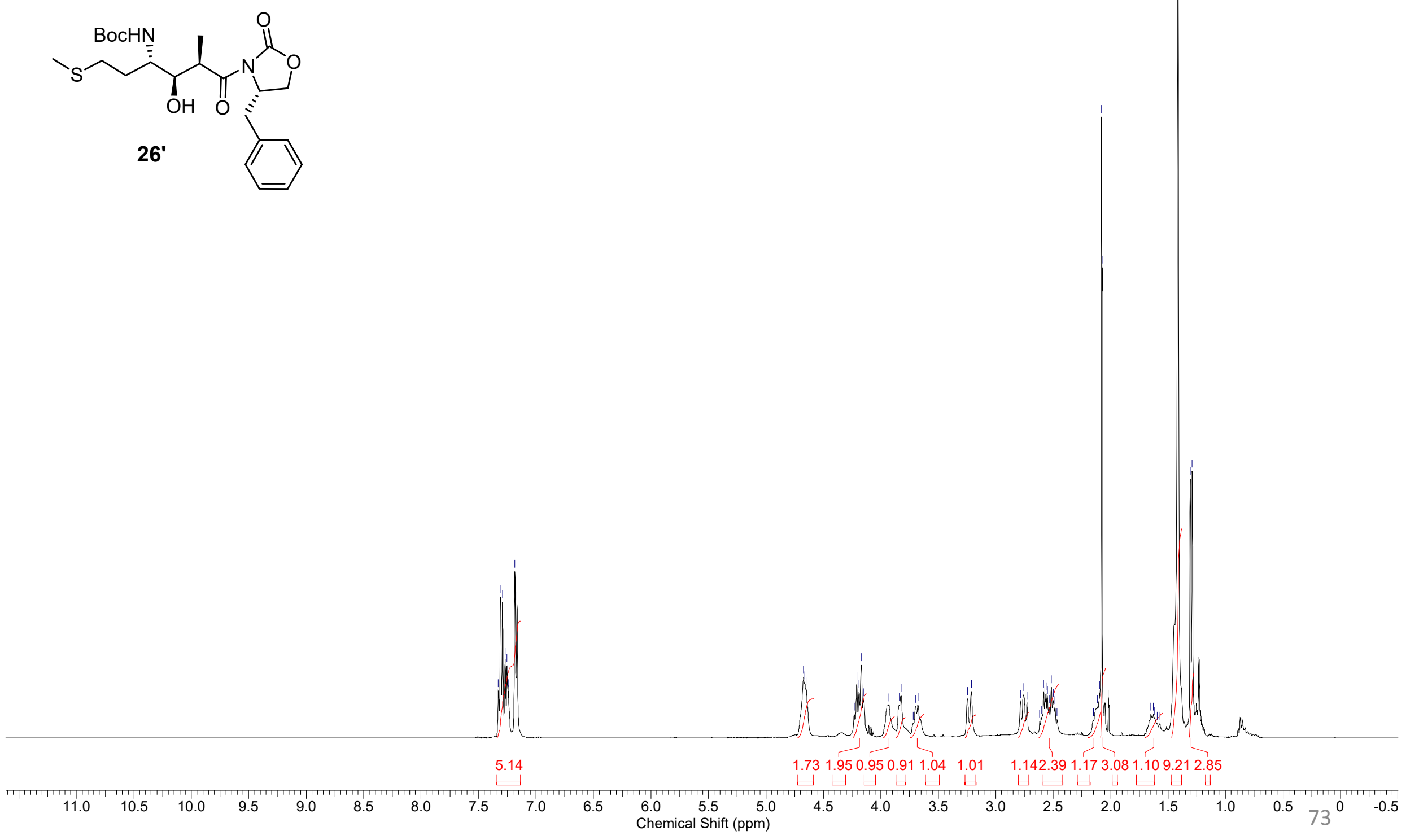


\section{${ }^{13} \mathrm{C}$ NMR (100 MHz, $\left.\mathrm{CDCl}_{3}\right)$ of compound 26'}

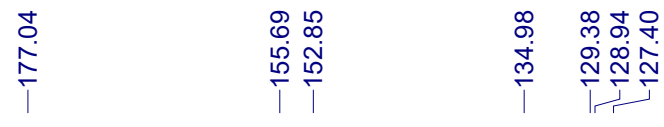

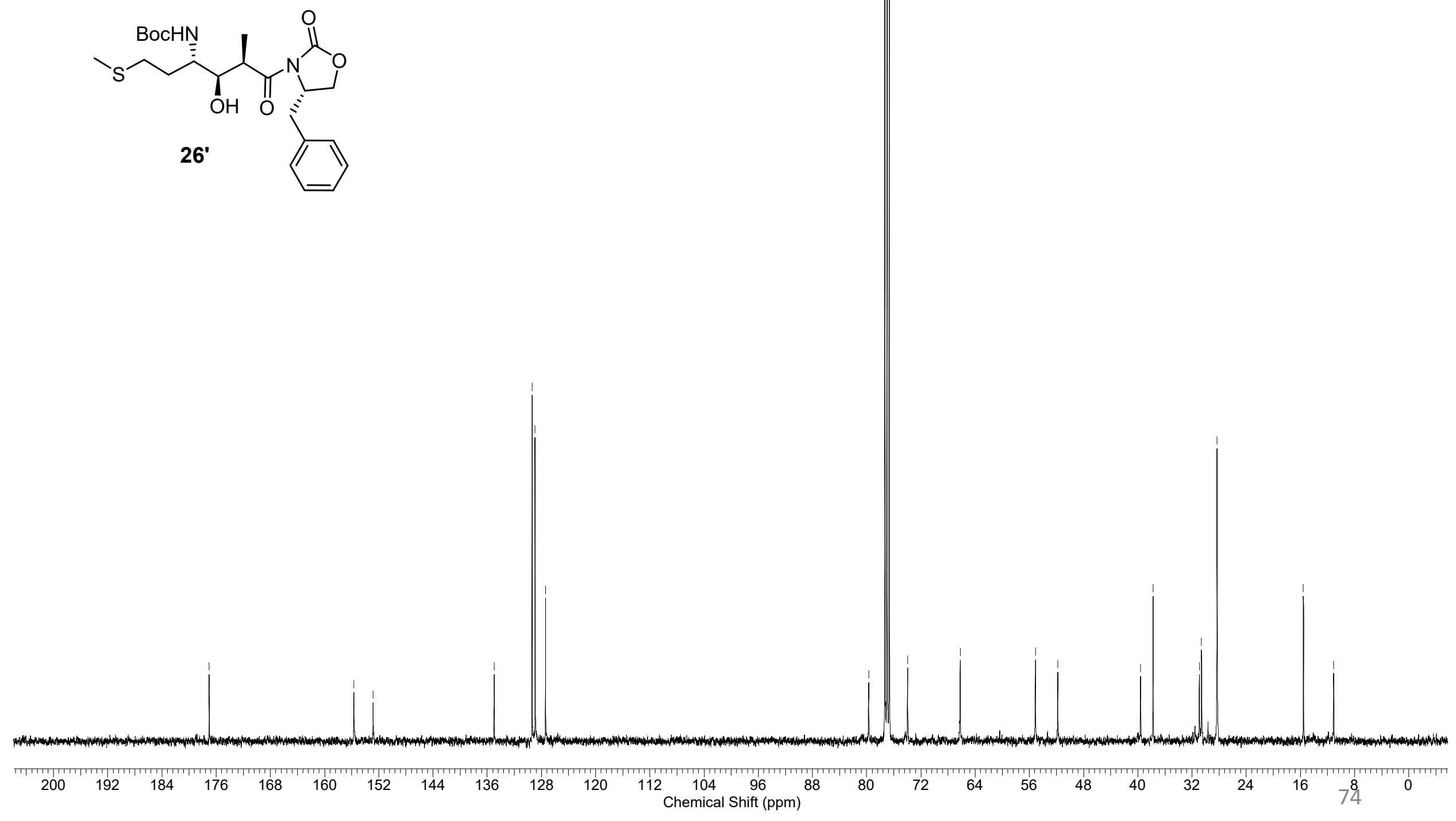

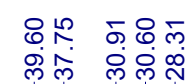

通 


\section{${ }^{1} \mathrm{H}$ NMR (400 MHz, $\mathrm{CDCl}_{3}$ ) of compound 27}
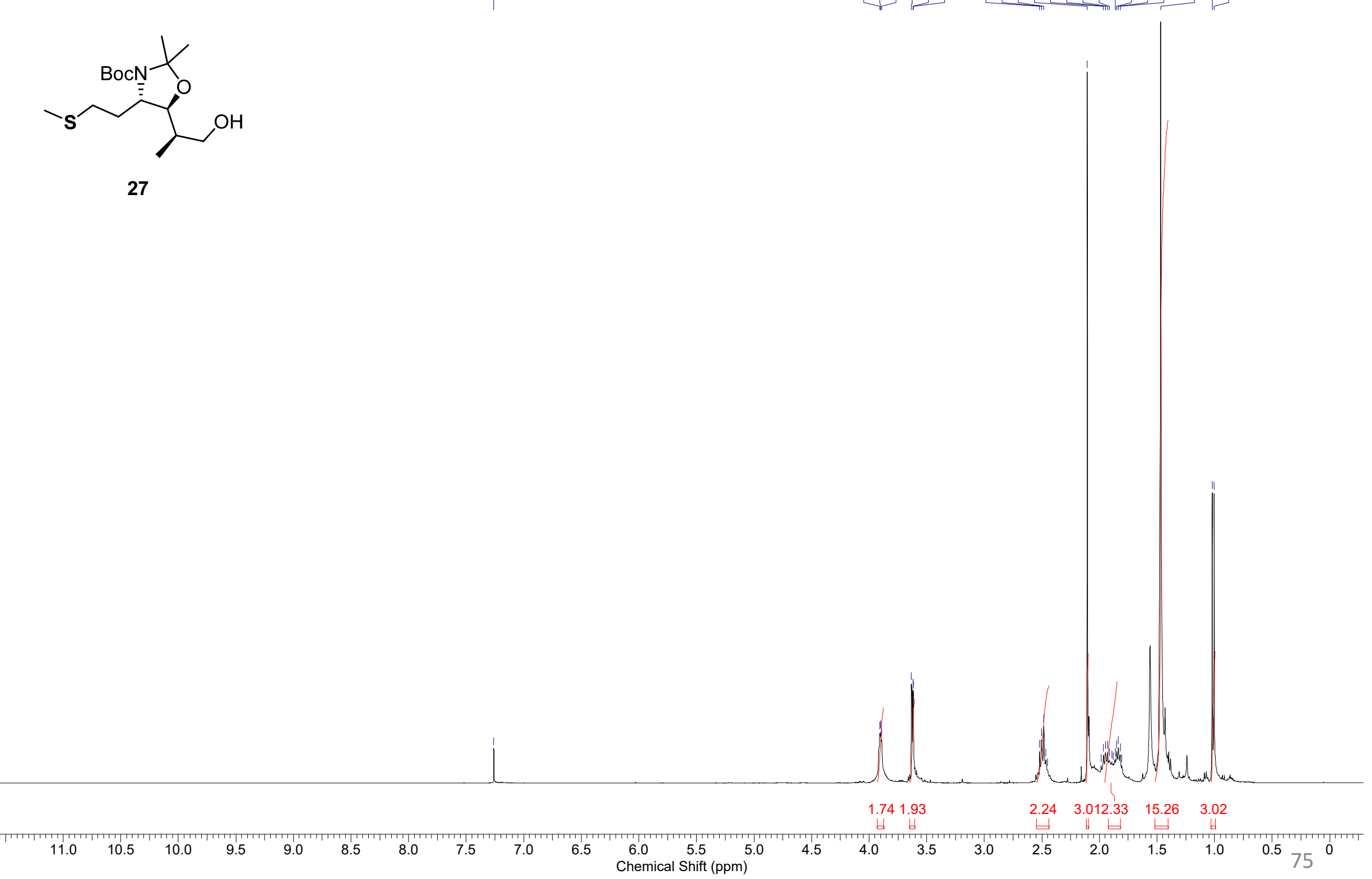
${ }^{13} \mathrm{C}$ NMR (100 MHz, $\left.\mathrm{CDCl}_{3}\right)$ of compound 27

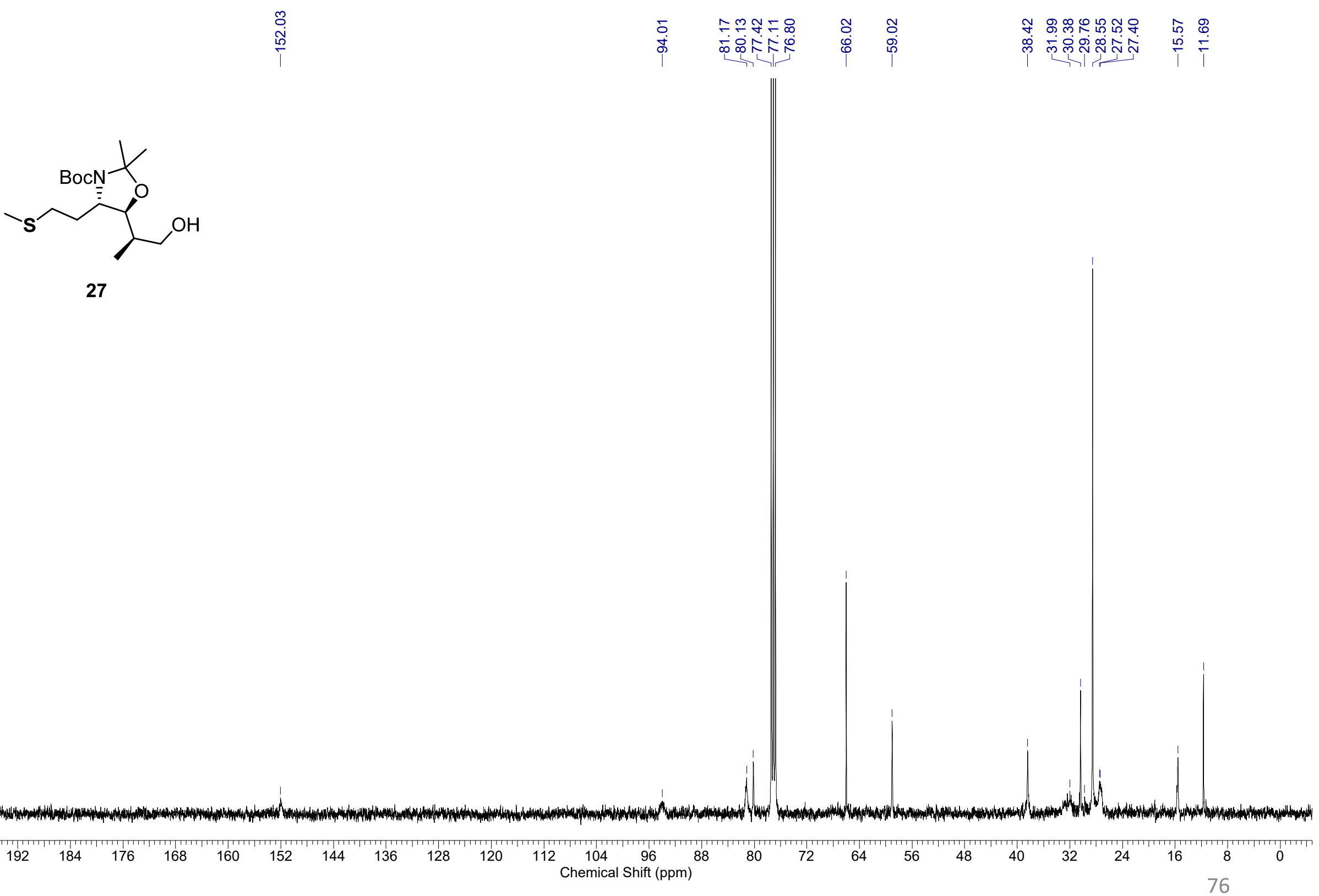


${ }^{1} \mathrm{H}$ NMR (400 MHz, $\mathrm{CDCl}_{3}$ ) of compound 27a

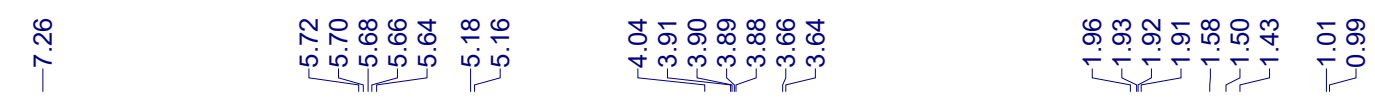

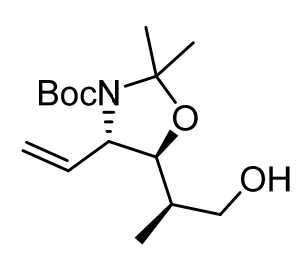

$27 a$

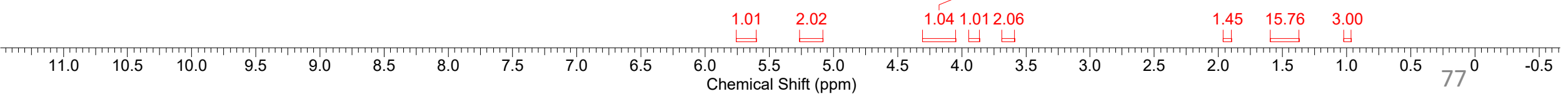




\section{${ }^{13} \mathrm{C}$ NMR (100 MHz, $\left.\mathrm{CDCl}_{3}\right)$ of compound 27a}

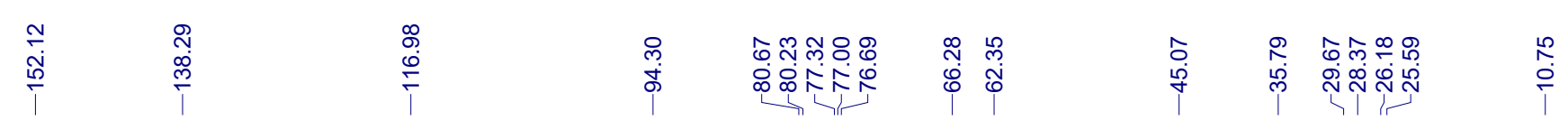

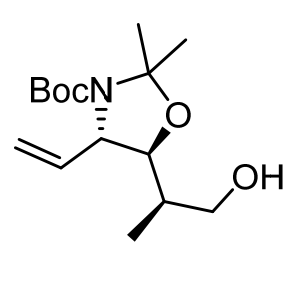

$27 a$

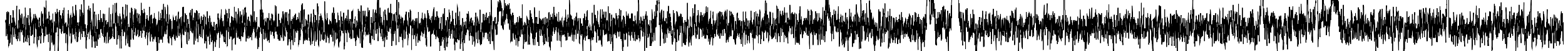

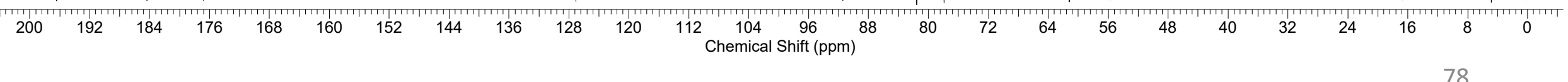




\section{${ }^{1} \mathrm{H}$ NMR (400 MHz, $\mathrm{CDCl}_{3}$ ) of compound 28}

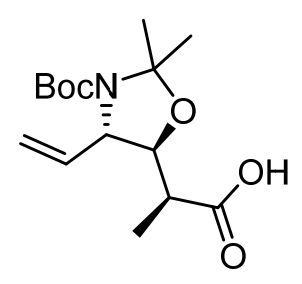

28
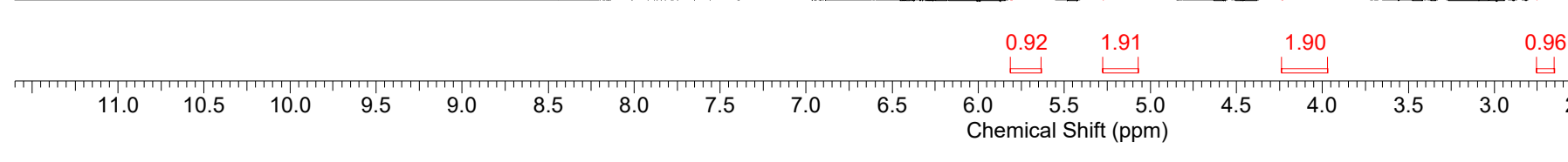

6.199 .293 .00
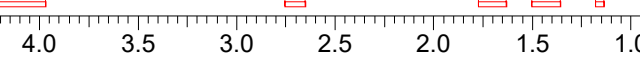
${ }^{13} \mathrm{C}$ NMR (100 MHz, $\left.\mathrm{CDCl}_{3}\right)$ of compound 28

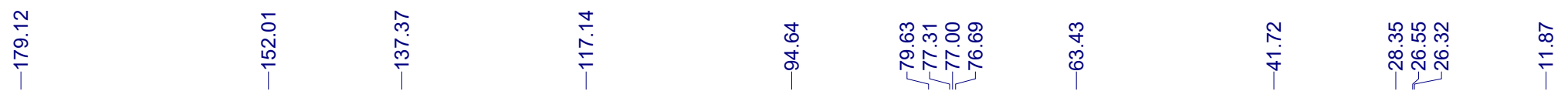

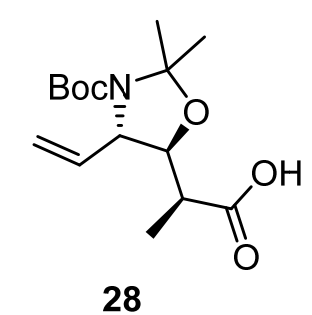

28

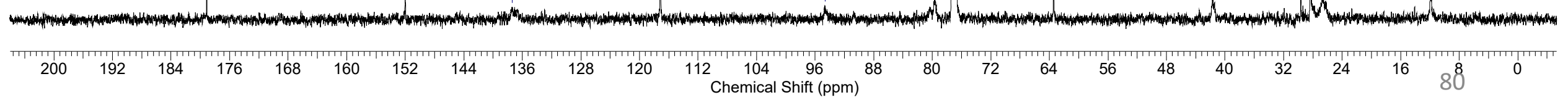




\section{${ }^{1} \mathrm{H}$ NMR (400 MHz, $\left.\mathrm{CD}_{3} \mathrm{OD}\right)$ of compound 29}

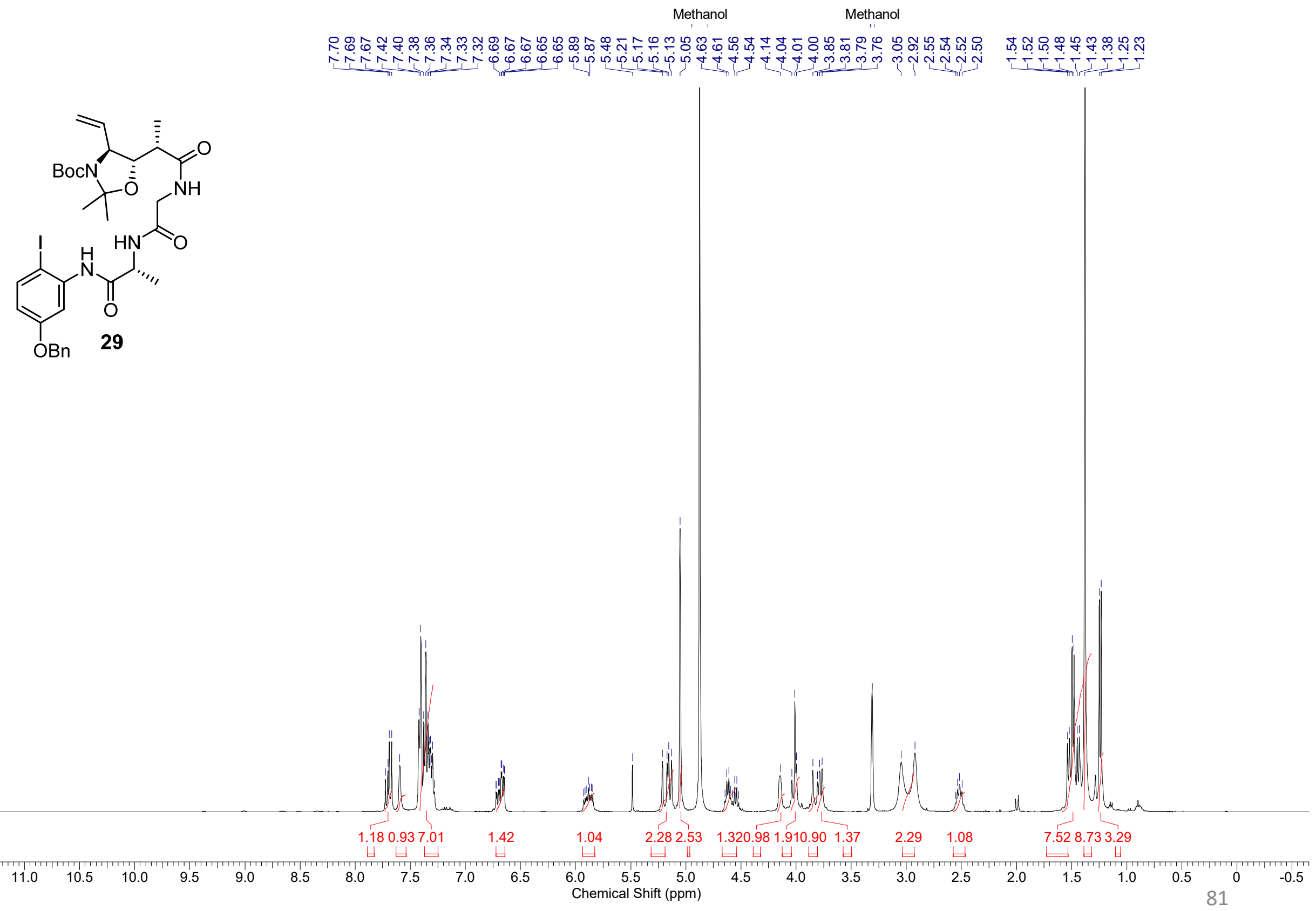




\section{${ }^{13} \mathrm{C}$ NMR (100 MHz, $\left.\mathrm{CD}_{3} \mathrm{OD}\right)$ of compound 29}

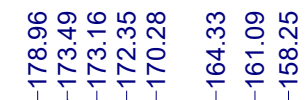

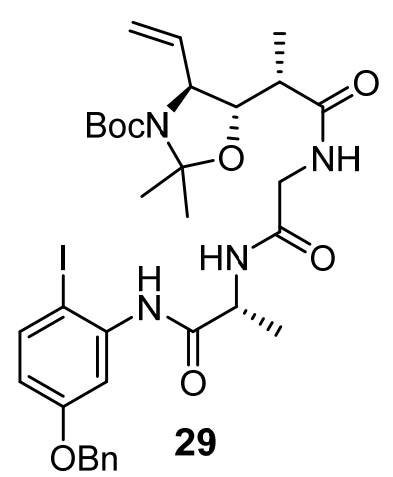

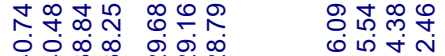

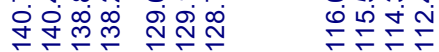

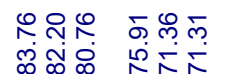

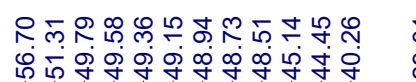

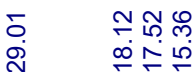

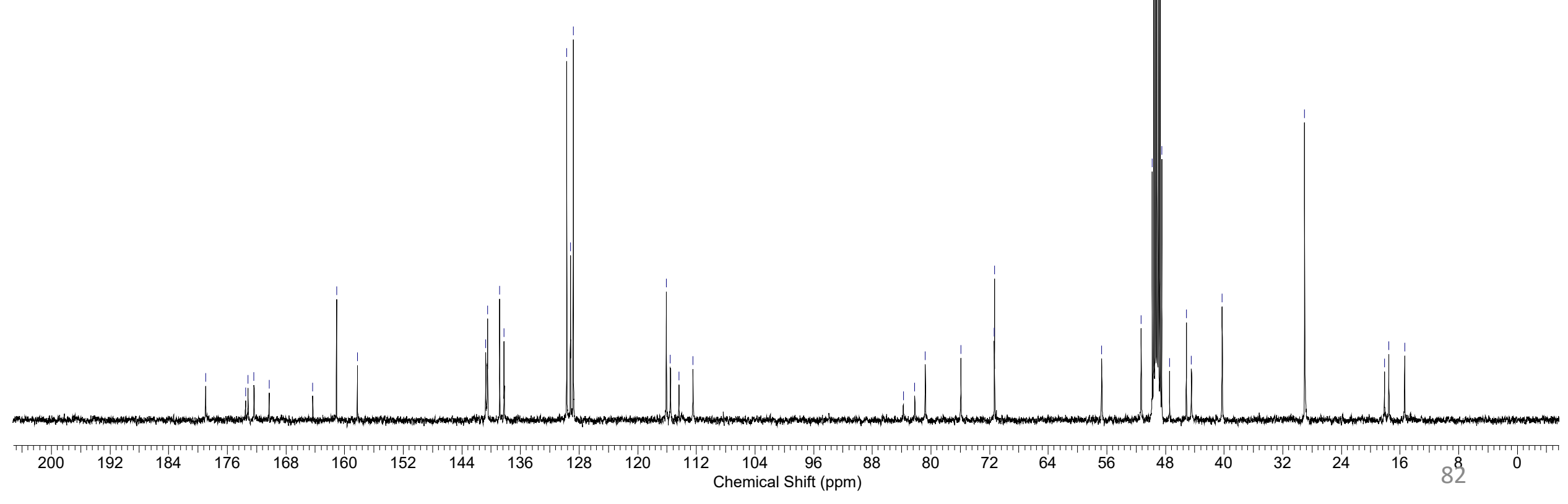


${ }^{1} \mathrm{H}$ NMR (400 MHz, CD $\left.\mathrm{CD}_{3} \mathrm{OD}\right)$ of compound 30

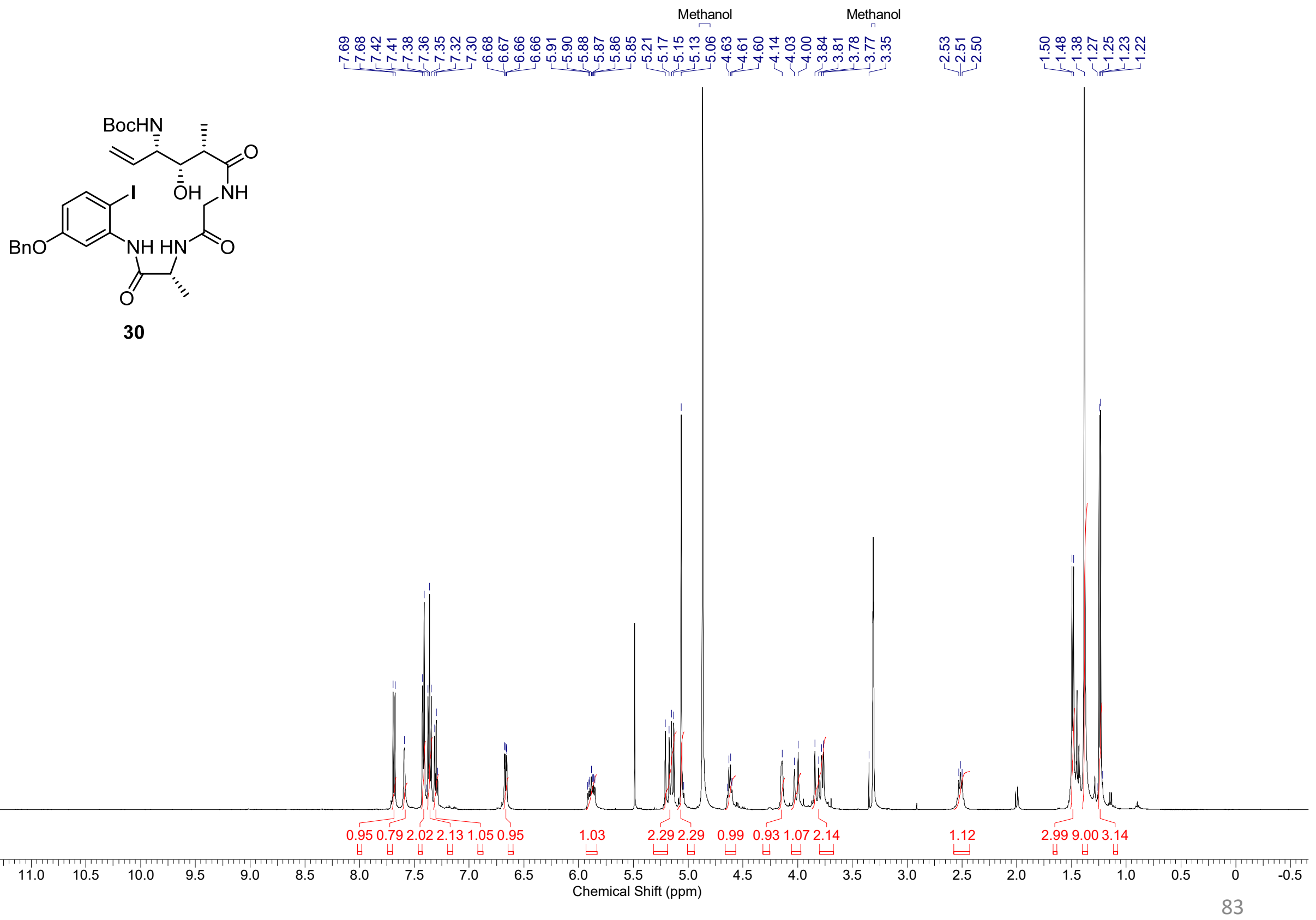




\section{${ }^{13} \mathrm{C}$ NMR (100 MHz, $\left.\mathrm{CD}_{3} \mathrm{OD}\right)$ of compound 30}

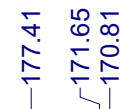

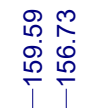

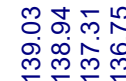

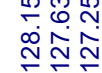

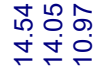

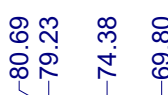

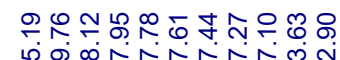
$\stackrel{\infty}{\stackrel{\infty}{N}}$

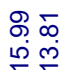

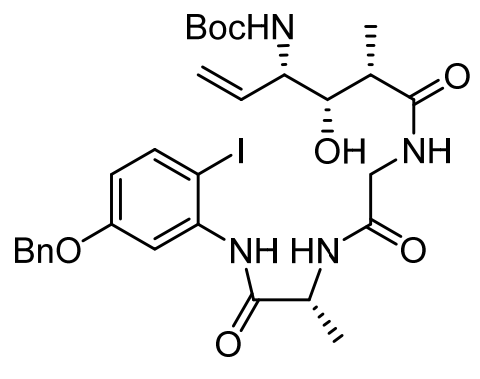

30

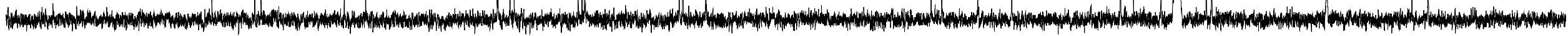

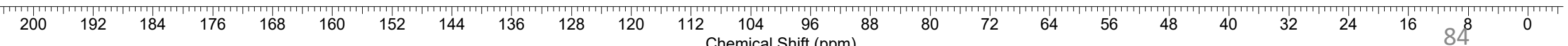




\section{${ }^{1} \mathrm{H}$ NMR (500 MHz, $\left.\mathrm{CD}_{3} \mathrm{OD}\right)$ of compound 31}

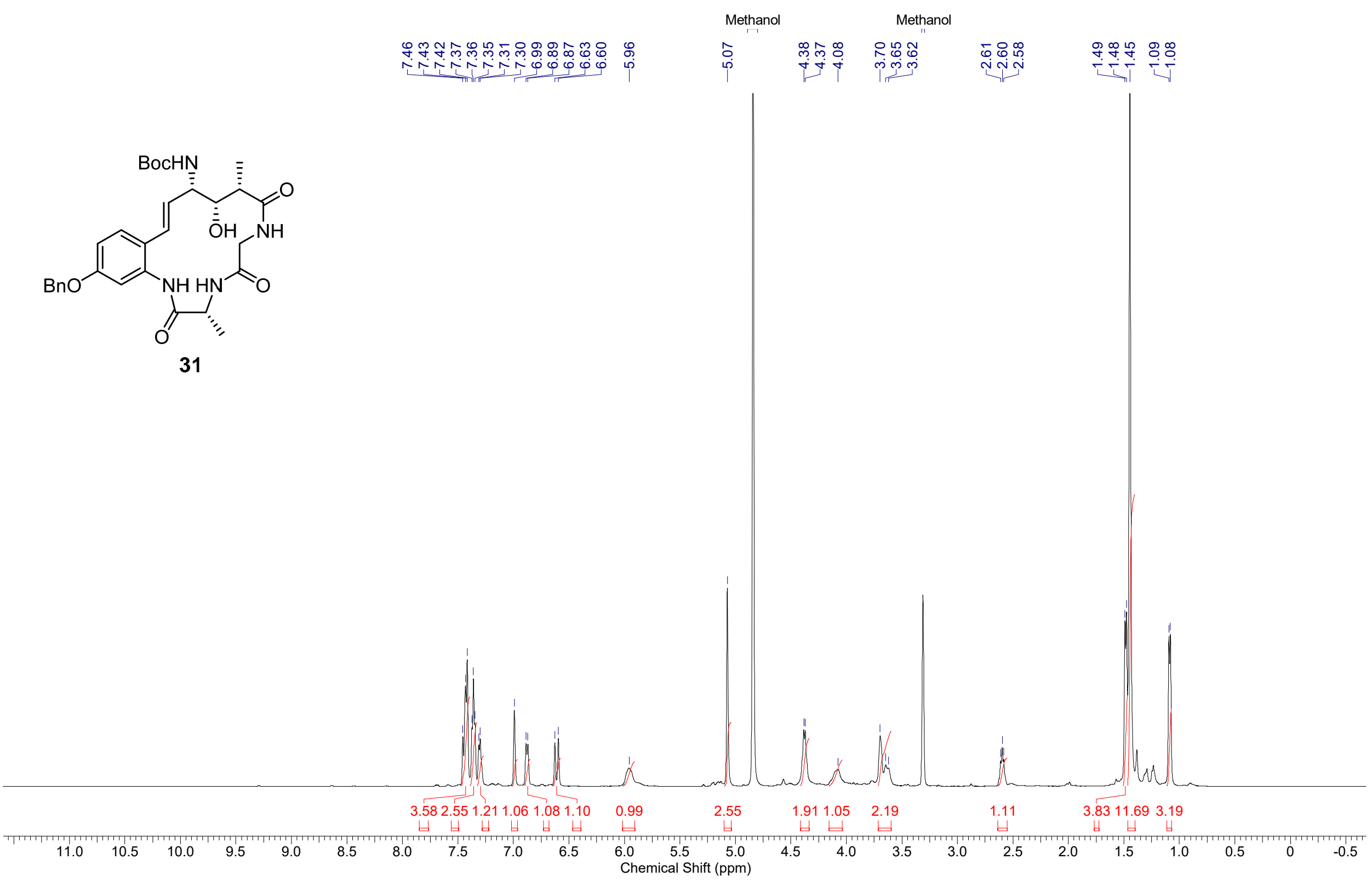




\section{${ }^{13} \mathrm{C}$ NMR (125 MHz, $\left.\mathrm{CD}_{3} \mathrm{OD}\right)$ of compound 31}

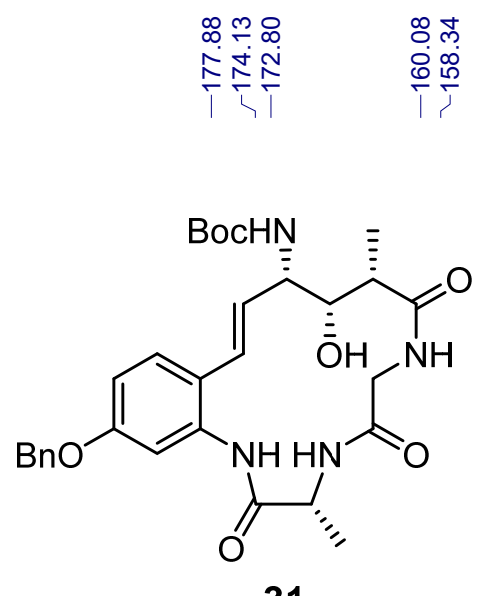

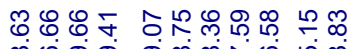

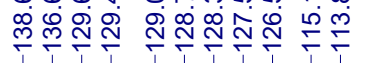

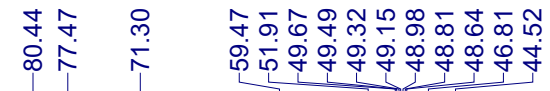

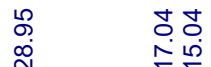

31

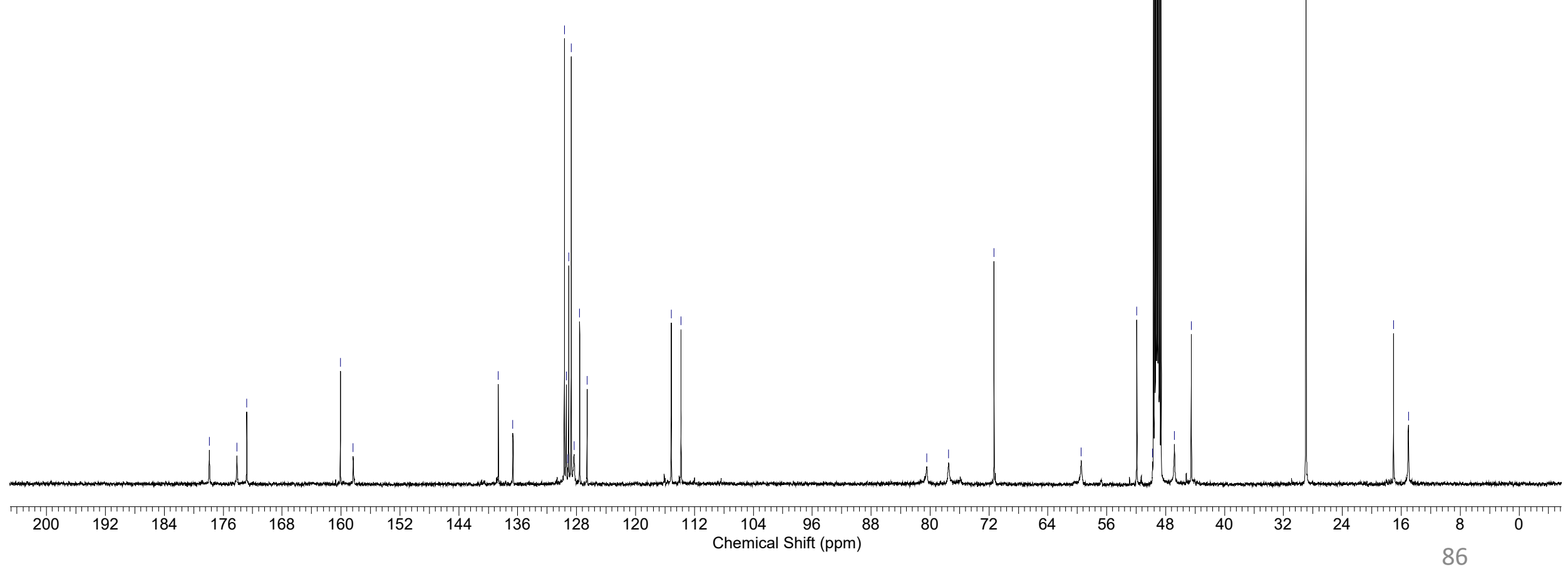




\section{${ }^{1} \mathrm{H}$ NMR (400 MHz, $\left.\mathrm{CD}_{3} \mathrm{OD}\right)$ of compound 32}

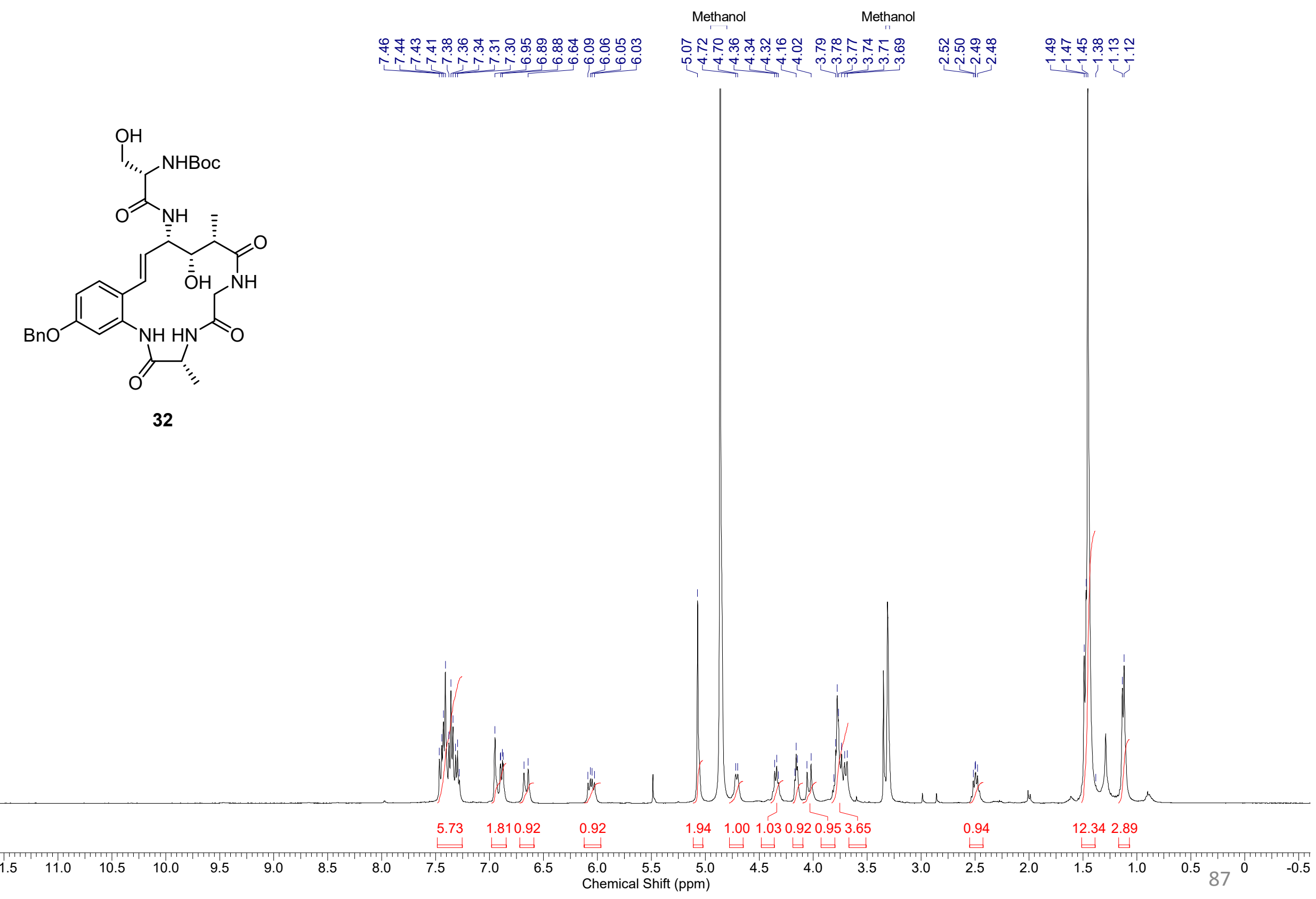




\section{${ }^{13} \mathrm{C}$ NMR (100 MHz, $\left.\mathrm{CD}_{3} \mathrm{OD}\right)$ of compound 32}

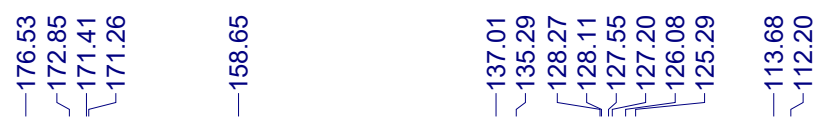

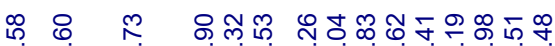

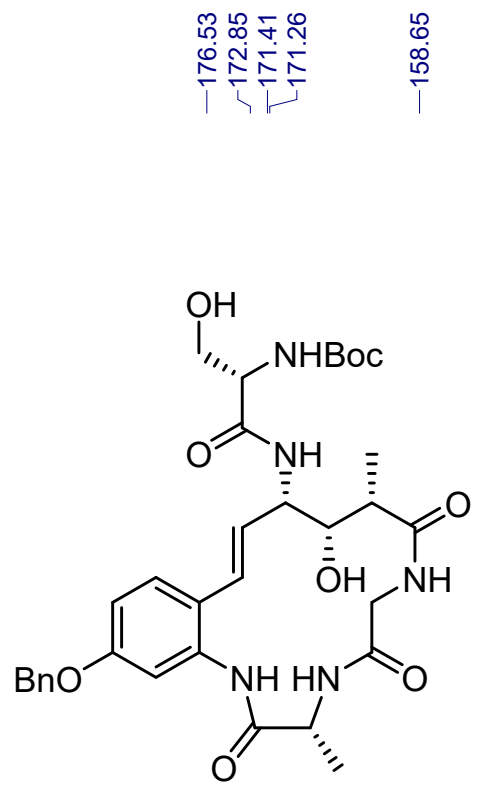

32

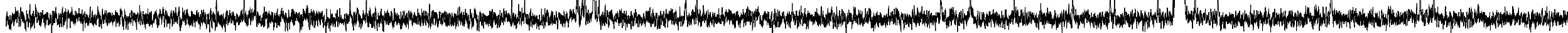

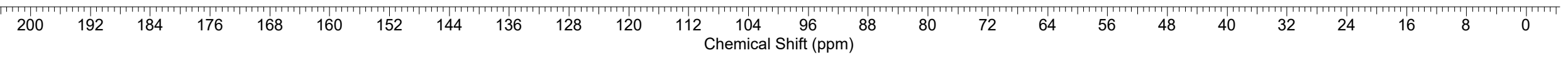




\section{${ }^{1}$ H NMR (500 MHz, DMSO-d 6 ) of compound 34}

$\stackrel{\stackrel{9}{ }}{\stackrel{一}{广}}$
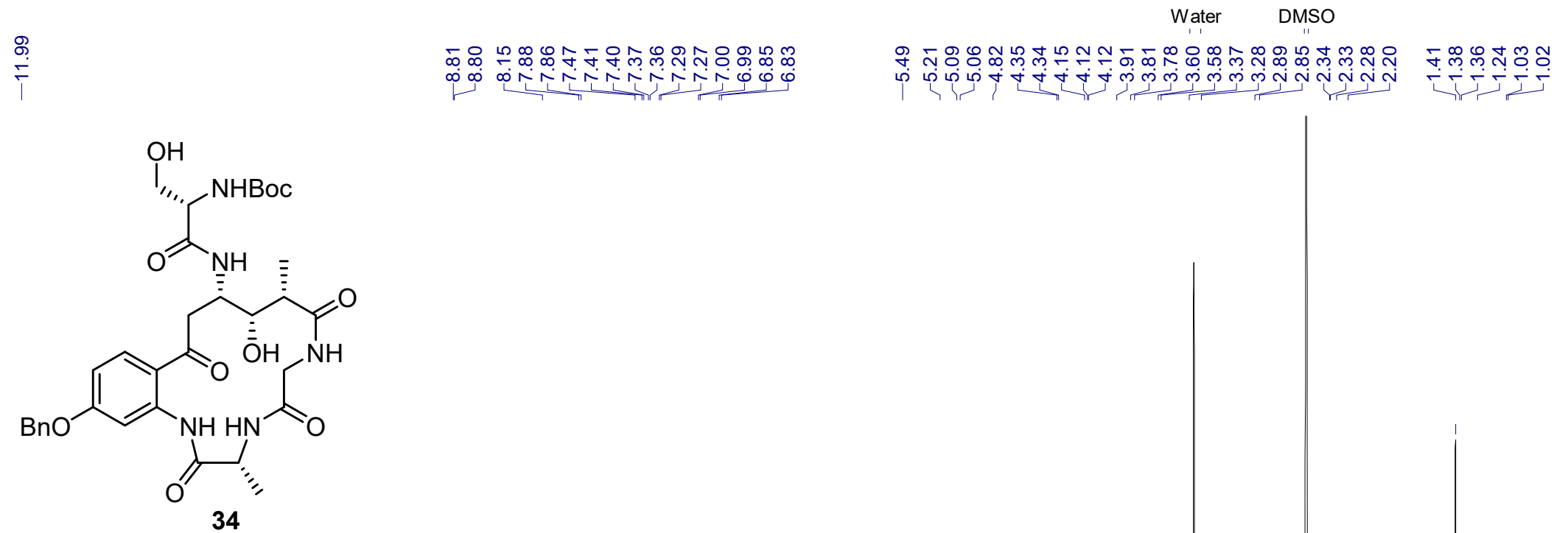

34 
${ }^{13} \mathrm{C}$ NMR (125 MHz, DMSO-d ${ }_{6}$ ) of compound 34

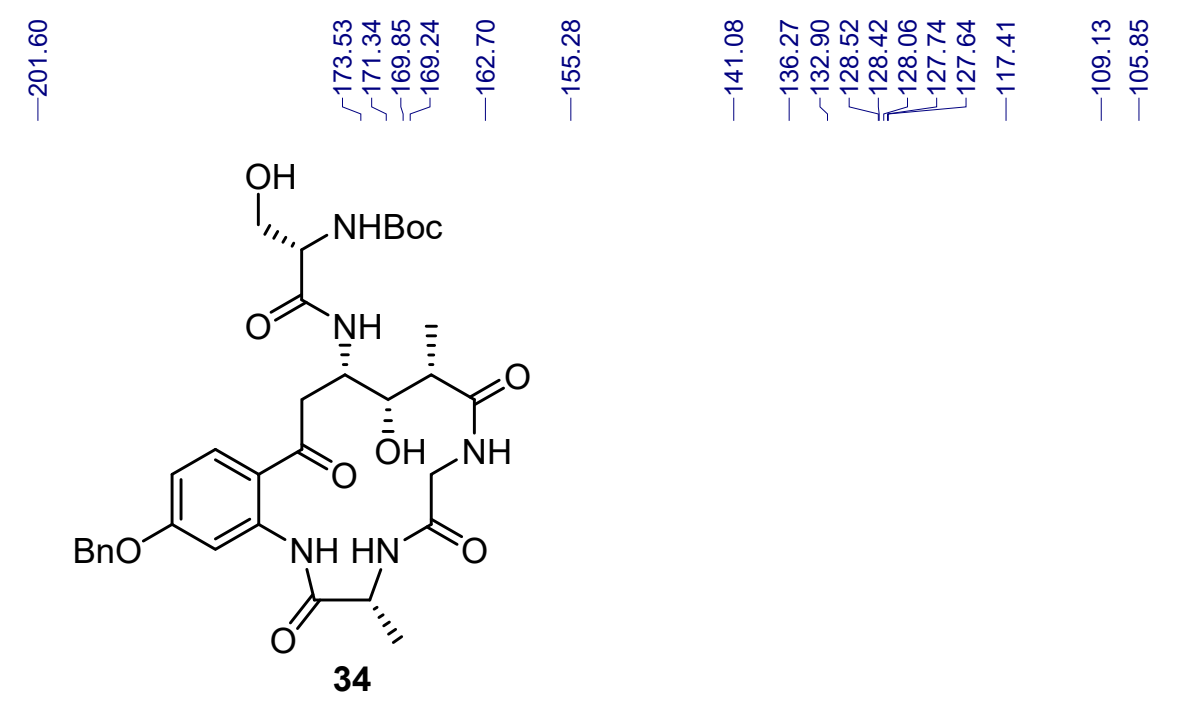

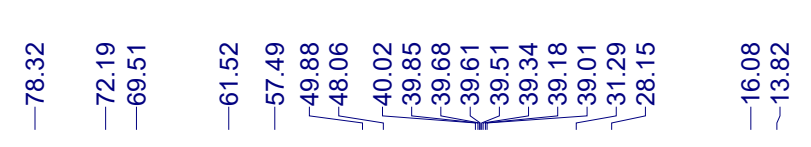




\section{${ }^{1}$ H NMR (500 MHz, DMSO-d ${ }_{6}$ ) of compound 35}
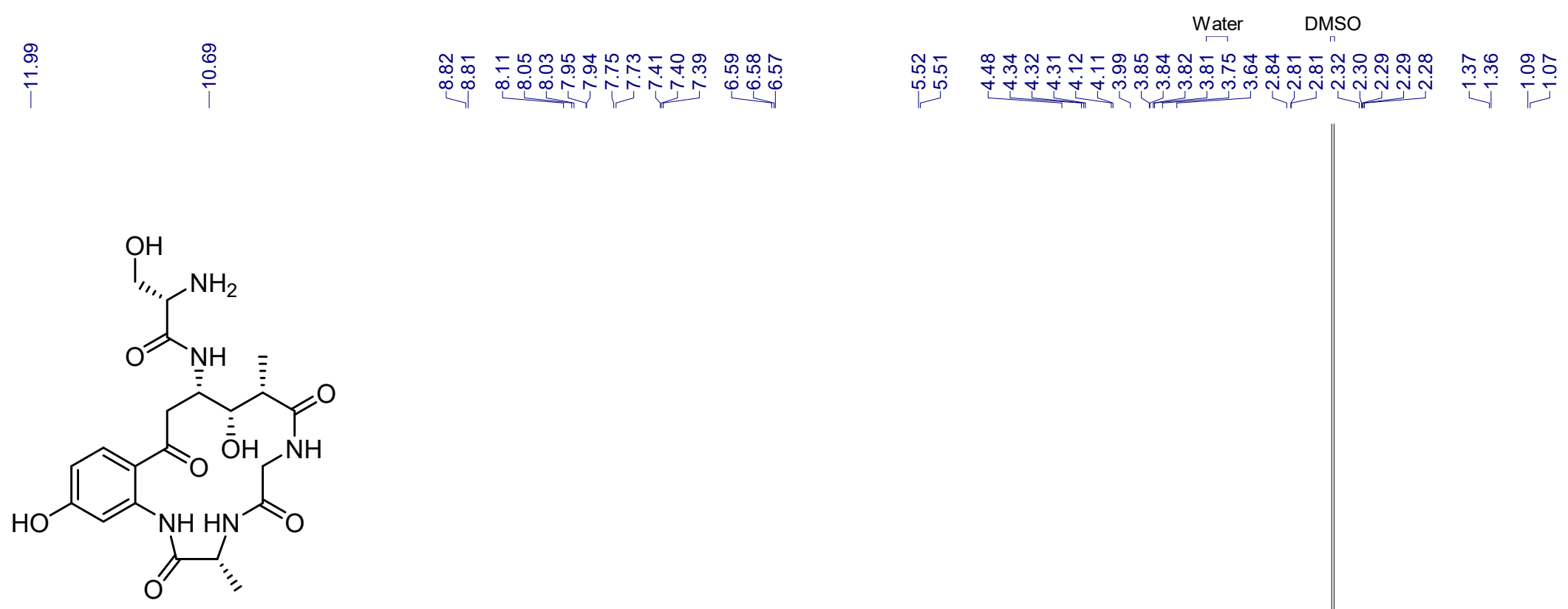

35

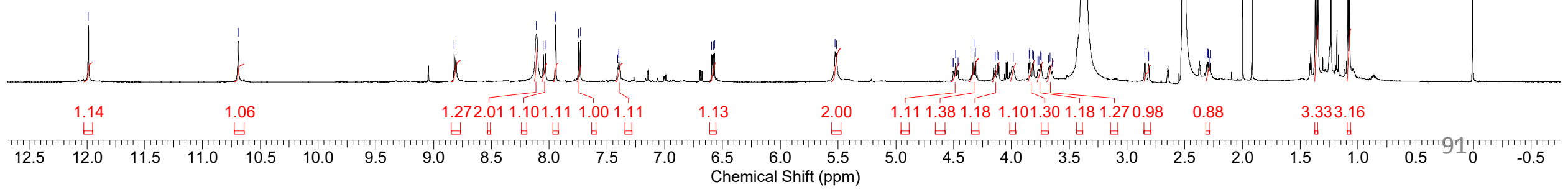


${ }^{13}$ C NMR (125 MHz, DMSO-d 6 ) of compound 35

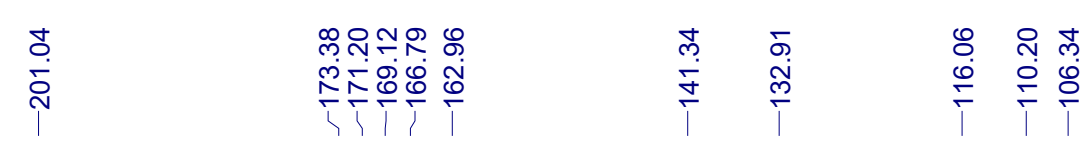

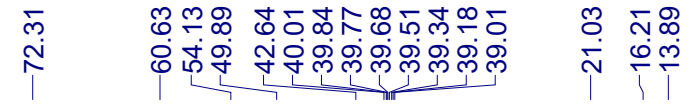

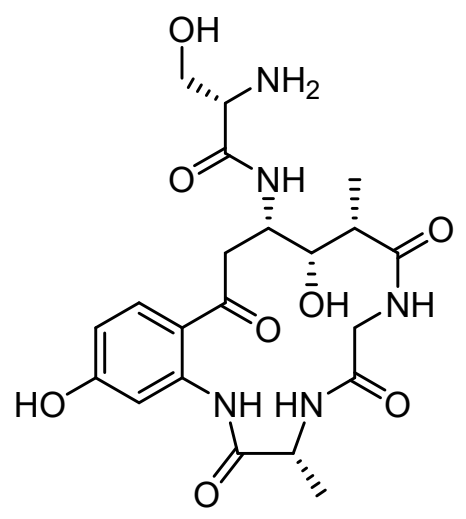

35 